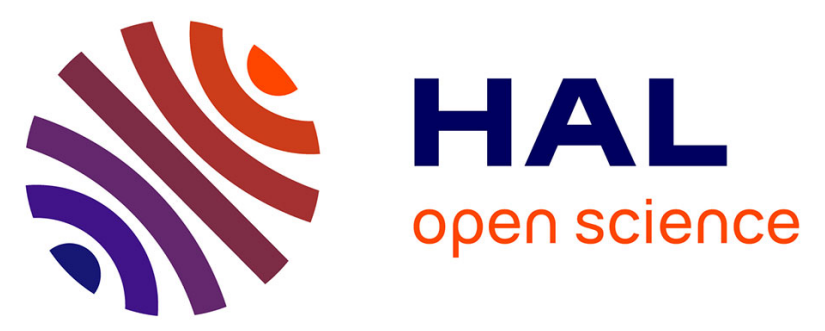

\title{
Une inhumation sous tumulus de l'âge du Bronze et une occupation de la fin du Néolithique à Gonesse, La Fosse aux Larrons (Val-d'Oise)
}

\author{
Jacques Tarrête, Vincent Krier, Jean-Jacques Puisségur, N.
}

Limondin-Lozouet, Chantal Leroyer, Gérard Firmin, Rose-Marie Arbogast, Henri Duday

\section{To cite this version:}

Jacques Tarrête, Vincent Krier, Jean-Jacques Puisségur, N. Limondin-Lozouet, Chantal Leroyer, et al.. Une inhumation sous tumulus de l'âge du Bronze et une occupation de la fin du Néolithique à Gonesse, La Fosse aux Larrons (Val-d'Oise). Gallia Préhistoire - Préhistoire de la France dans son contexte européen, 2012, 54, pp.239-289. 10.3406/galip.2012.2496 . hal-01485844

\section{HAL Id: hal-01485844 \\ https://hal.science/hal-01485844}

Submitted on 19 Dec 2019

HAL is a multi-disciplinary open access archive for the deposit and dissemination of scientific research documents, whether they are published or not. The documents may come from teaching and research institutions in France or abroad, or from public or private research centers.
L'archive ouverte pluridisciplinaire HAL, est destinée au dépôt et à la diffusion de documents scientifiques de niveau recherche, publiés ou non, émanant des établissements d'enseignement et de recherche français ou étrangers, des laboratoires publics ou privés.

\section{(ㅇ)(1) $\$$}

Distributed under a Creative Commons Attribution - NonCommercial - NoDerivatives $\mid 4.0$ 


\title{
Une inhumation sous tumulus de l'Âge du Bronze et une occupation de la fin du Néolithique à Gonesse, La Fosse aux Larrons (Val-d'Oise)
}

\section{Bronze Age tumulus inhumation and Late Neolithic occupation at La Fosse aux Larrons (Gonesse, Val-d'Oise, France)}

\author{
Jacques TArRête ${ }^{1}$ et Vincent Krier ${ }^{2}$, coordinateurs, Jean-Jacques PuisséGur (t) \\ et Nicole Limondin-Lozouet ${ }^{3}$, Chantal Leroyer ${ }^{4}$ et Gérard Firmin ${ }^{5}$, \\ Rose-Marie Arbogast ${ }^{6}$, Henri DudaY ${ }^{7}$
}

Mots clé. Âge du Bronze ancien/moyen, Néolithique final, études environnementales, ${ }^{14} \mathrm{C}$, tumulus, inhumation, céramique, lithique, faune.

\begin{abstract}
Résumé. Découvert fortuitement en 1976 suite à un décapage industriel, un tumulus à couronne de pierres comportant une inhumation centrale a fait l'objet d'une fouille de sauvetage. Situé dans la vallée du Crould au nord de Paris, les diverses études environnementales (géoarchéologie, malacologie, palynologie) ont permis de restituer l'évolution du paysage durant le Subboréal et I'insertion des occupations humaines successives. La stratigraphie révèle une première implantation caractérisée par une couche archéologique partiellement conservée comportant des vestiges céramiques, lithiques et des restes de faune. Des artefacts de même nature se retrouvent dans la constitution du tumulus édifié ultérieurement en partie au détriment de cette occupation initiale. L'étude du monument montre en effet un creusement périphérique destiné à collecter des sédiments pour former un noyau central ceinturé d'une couronne de pierres recouverte d'une chape de terre elle-même surmontée d'un mince niveau organique. Au centre se trouvait un inhumé de sexe masculin dépourvu de mobilier, objet d'une étude anthropologique détaillée. Les datations ${ }^{14} \mathrm{C}$, les analyses environnementales et l'examen du mobilier concordent pour situer avec vraisemblance les restes d'habitat au début du Néolithique final et l'édification du tumulus à la charnière de l'Âge du Bronze ancien et moyen.
\end{abstract}

Keywords. Early Bronze Age, Middle Bronze Age, Final Neolithic, environmental studies, ${ }^{14} \mathrm{C}$ dates, inhumation, pottery, lithic industry, fauna.

\begin{abstract}
A rescue excavation was launched after a tumulus with a stone lining, containing a central inhumation was accidentally exposed by construction work in the Crould valley, north of Paris, in 1976. The various environmental studies (geoarchaeology, malacology, palynology) made it possible to reconstruct the evolution of the landscape during the Subboreal in relation with successive human occupations. Stratigraphy shows that the first occupation is characterized by a partially preserved archaeological layer containing pottery, lithic and faunal remains. Artifacts of the same nature were found in the tumulus, the construction of which had partly destroyed the earlier occupation. The study of the monument revealed that a circular ditch was used to provide sediments for a central core, surrounded by a lining of stones covered by a layer of soil, itself overlain by a thin organic level. The centre of the monument contained a male burial with no grave goods. A detailed anthropological analysis was undertaken of this individual. According to ${ }^{14} \mathrm{C}$ dates, environmental analyses and the study of the artifacts, the occupation most likely dates to the beginning of Final Neolithic and the building of the tumulus to the transition between the Early and Middle Bronze Age.
\end{abstract}

Translation: Thierry Donnadier

1. Ministère de la culture et UMR7041 ArScAn, Éthnologie préhistorique, Maison René-Ginouvès Nanterre (jacques.tarrete@mae.u-paris10.fr)

2. INRAP, direction interrégionale Centre-île-de-France, PANTIN (vincent.krier@culture.fr)

3. CNRS UMR 8591, Laboratoire de Géographie Physique, Meudon (Nicole.Limondin@cnrs-bellevue.fr)

4. MCC/U.M.R6566 CReAAH, Université de Rennes 1, RENNES (chantal.leroyer@univ-rennes1.fr)

5. 49 Avenue Lazare Carnot, 21340 NOLAY

6. CNRS-UMR7044, MISHA, Strasbourg (rose-marie.arbogast@misha.cnrs.fr)

7. PACEA, UMR5199, Université de Bordeaux 1, Laboratoire d'anthropologie des populations du passé (h.duday@anthropologie.u-bordeaux1.fr) 


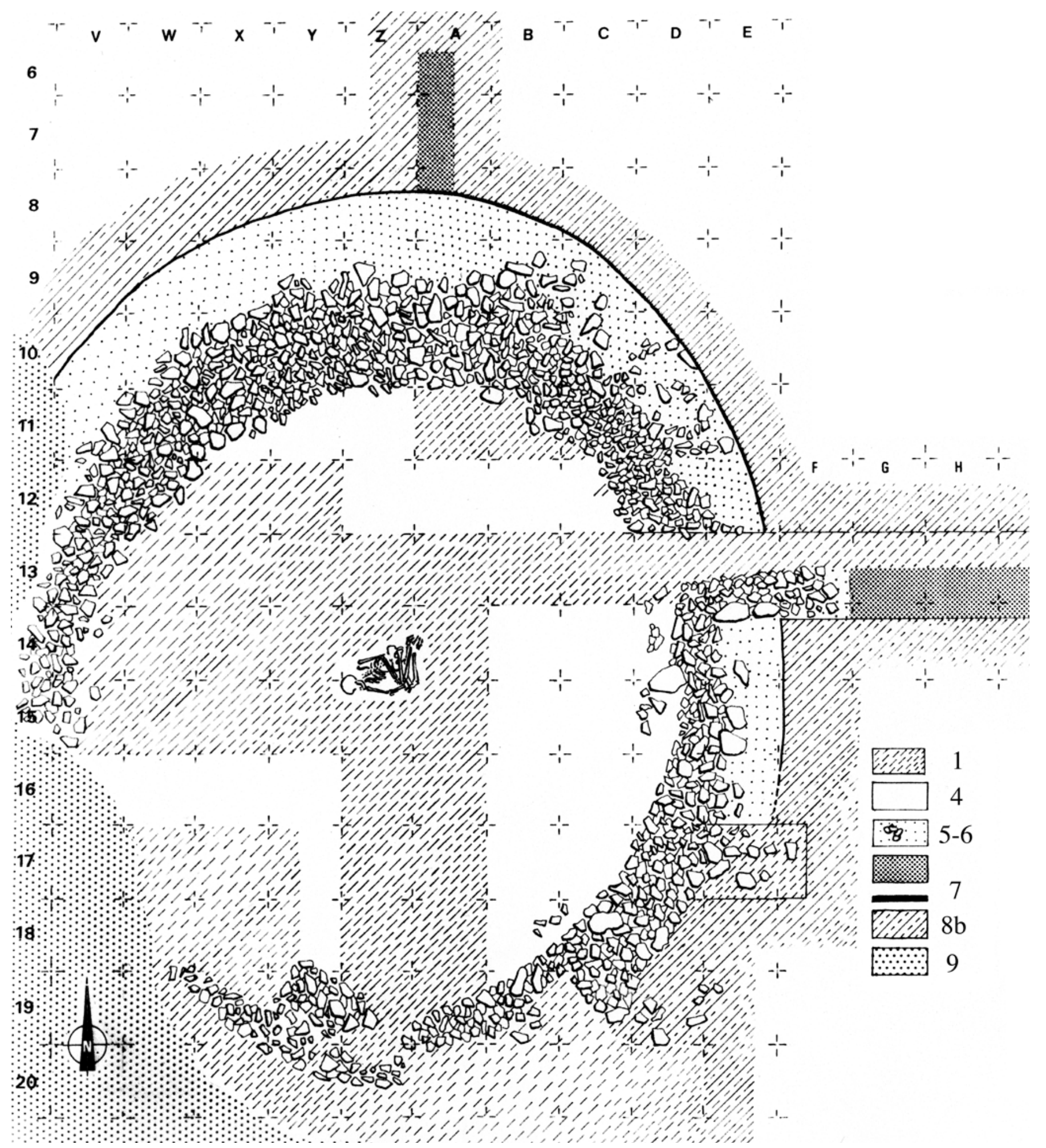

Fig. 1 - Gonesse (Val-d'Oise), La Fosse aux Larrons : plan général du site en fin de fouille, les cartouches désignent, de haut en bas : 1, tuf en place (couche 1) ; 2 , sommet de la couche 4, zones non fouillées; 3 , couches 5 et $6 ; 4$ et 5,couche $7 ; 6:$ couche $8 b$;

7, remblai actuel (couche 9).

Gonesse (Val-d'Oise), La Fosse aux Larrons : site plan at the end of the excavation. 1: tuff subsoil (layer 1); 2: top of layer 4, unexcavated zones; 3: layers 5 and 6; 4 and 5: layer 7; 6 : layer 8b; 7: modern backfill (couche 9). 


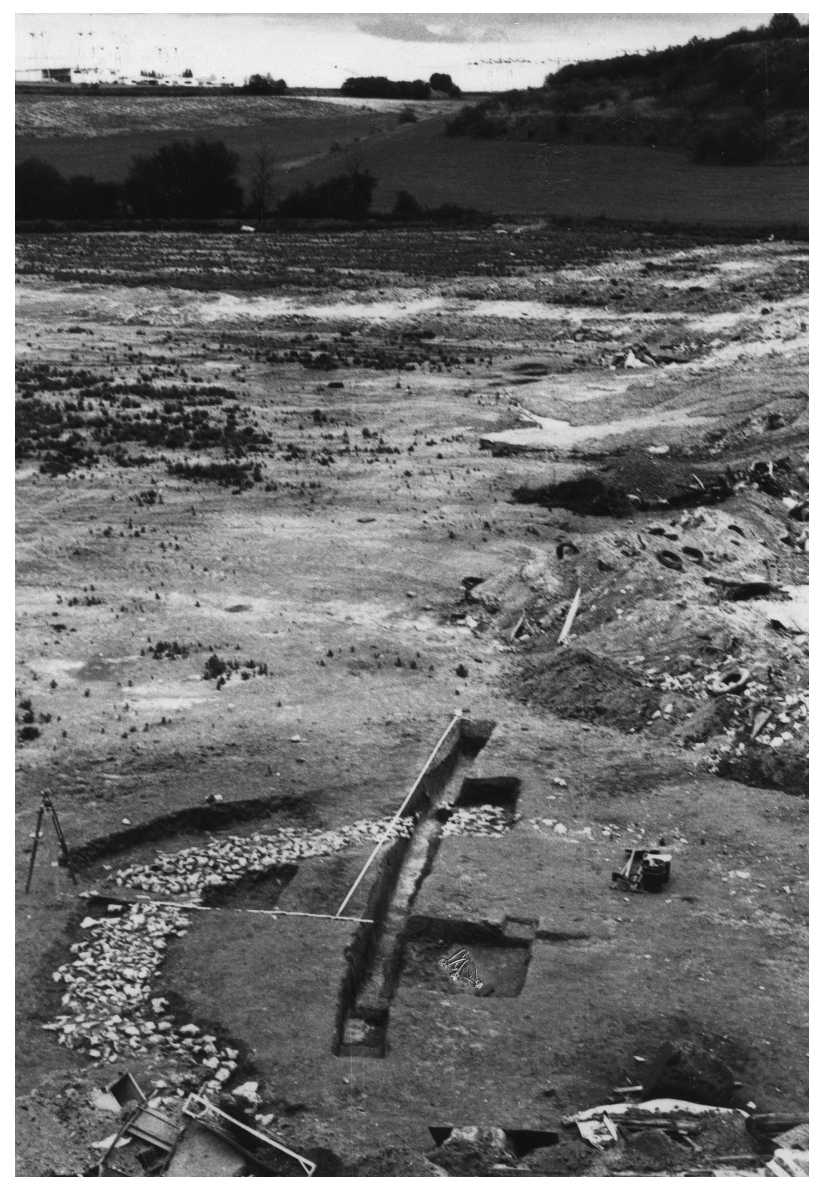

\section{AVANT-PROPOS}

Il n'est pas très aisé de publier une fouille qui s'est déroulée il y a plus de 30 ans (Brézillon, 1977), d'autant plus lorsqu'une partie des analyses a été réalisée peu de temps après : c'est le cas de l'étude anthropologique qu'on trouvera ici quasiment telle qu'Henri Duday l'avait adressée en septembre 1984 et qu'il a bien voulu nous laisser reproduire, de l'analyse malacologique réalisée dès février 1977 par l'abbé Jean-Jacques Puisségur, décédé depuis, et que Nicole Limondin, l'une de ses disciples, a très volontiers, à notre demande, adaptée aux normes actuelles et du rapport détaillé de l'analyse palynologique rédigé par Gérard Firmin cette même année que Chantal Leroyer a bien voulu revoir à l'aune des évolutions récentes de cette discipline. Une première tentative de mise en route de cette publication avait été amorcée en 1999 grâce au concours de Rose-Marie Arbogast qui avait effectué un premier décompte de la faune, récemment complété par un commentaire interprétatif, ainsi que la sélection d'ossements animaux pour une datation $14 \mathrm{C}$ sur le niveau présumé d'occupation précédant l'édification du tumulus ; de plus, une analyse géo-archéologique avait alors été réalisée par Vincent Krier qui avait été à l'époque l'un des fouilleurs bénévoles du site et qu'il a récemment revue. Que tous ici soient remerciés pour leur aide permettant enfin la parution des résultats de cette " fouille de sauvetage ", en hommage à la mémoire de Michel Brézillon qui a marqué notre discipline et nombre de ses pratiquants et qui méritaient d'être connus au moins par leur singularité en région Île-de-France.
Fig. 2 - Gonesse (Val-d'Oise), La Fosse aux Larrons : le site archéologique dans son contexte; la sépulture,

démontée à ce stade de la fouille, a été schématiquement restituée.

Gonesse (Val-d'Oise), La Fosse aux Larrons: the archaeological site in its context; the grave, removed at this stage of the excavation, is reproduced schematically.

\section{CIRCONSTANCES ET DÉROULEMENT DE L'INTERVENTION}

À une vingtaine de kilomètres au nord de Paris, le site fut découvert avant l'été 1976 par des membres de groupes archéologiques locaux («Les Amis de Gonesse » et « Jeunesses préhistoriques et géologiques de France, section de Villiers-le-Bel ») à la suite d'un décapage mécanique précédant l'extension d'une décharge au lieu-dit La Fosse aux Larrons sur la commune de Gonesse, Val-d'Oise (section ZH, parcelle $45 ; \mathrm{x}=609,574$; $\mathrm{y}=1143,500 ; \mathrm{z}=53 \mathrm{~m}$ ). Interprété dans un premier temps comme les restes d'un moulin, les fouilles pratiquées alors par ces équipes consistèrent en une succession de sondages formant une tranchée discontinue est-ouest recoupant une couronne de pierres (cf. fig. 1, plan général, bande 13). La présence de vestiges d'industrie lithique sur et sous la structure incita à prévenir à l'automne le directeur des antiquités préhistoriques qui identifia l'existence du tumulus. Encadré par le personnel de la direction (Michel Brézillon, Jacqueline Degros, Jacques Tarrête et JeanMichel Cointin qui réalisa par la suite l'ensemble de l'illustration récemment reprise en DAO par Maurice Hardy de l'UMR 7041 et, pour la céramique, par Daniel Simonin du musée de préhistoire de Nemours) avec le concours d'une douzaine de bénévoles et étudiants (Michel Cecchini, Catherine Girard-Farizy, Vincent Krier, Jean Leclerc, Dominique Legoupil, Roland Mathieu, François Moser, Nicole Pigeot, Bernard Rochon, Frédéric Rymarckzyk, Alain Sueur) qui totalisèrent 78 journées de travail sur le terrain, l'opération se déroula du 11 au 15 octobre puis du 28 octobre au ler novembre pour un coût, hors salaires, d'un peu plus de $1500 \mathrm{~F}$. Celle-ci fut limitée d'une part par des conditions atmosphériques peu favorables et d'autre part en raison de l'environnement particulier des vestiges (fig. 2), décharge industrielle d'au moins $20 \mathrm{~m}$ de hauteur au sud-est et décharge ménagère d'une dizaine de mètres de hauteur à l'ouest condamnant partiellement la structure (cf. fig. 1, plan général, bande U 10 à 13, bandes V-X 16 à 20) : on peut toutefois estimer les terres traitées à un volume de $50 \mathrm{~m} 3$. Rappelons enfin que ce qui prévalait alors dans un sauvetage était la rapidité de l'intervention pour une compréhension la plus exhaustive possible des caractéristiques majeures du site fouillé. Aussi les secteurs de fouille ont-ils été définis en fonction de la densité d'apparition des vestiges comme de la possibilité matérielle d'y accéder : le secteur sud-ouest a ainsi été délaissé car d'une part trop proche voire oblitéré par la décharge et d'autre part parce que la fouille de certains carrés (X-Y/ 17-18) montrait leur extrême pauvreté à tous niveaux.

\section{PREMIÈRES OBSERVATIONS ET ENJEUX DE L'ÉTUDE}

L'examen initial sur le terrain le 23 septembre 1976 permit d'observer au sud de la ligne de sondages un assemblage 


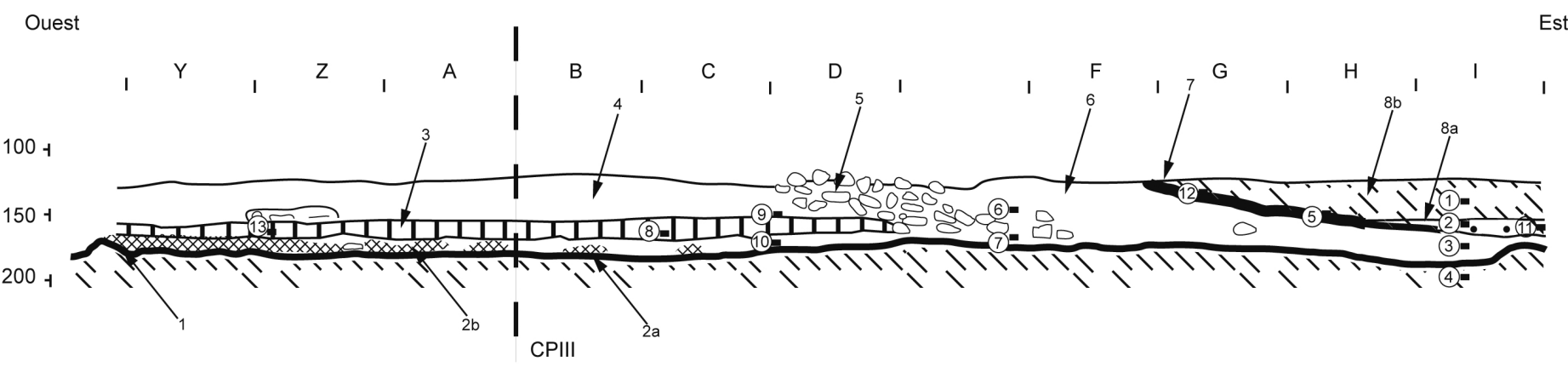

Fig. 3 - Gonesse (Val-d'Oise), La Fosse aux Larrons : coupe ouest-est (CP I) à la limite des bandes 12 et 13 (cotes altimétriques en cm sous le niveau relatif de chantier) avec l'emplacement des prélèvements pour les analyses palynologique $(1$ à 8,10 et 13) et malacologique (9 à 12, le prélèvement 10 ayant été dédoublé) : 1, tuf ; 2a, limon gris à horizon gris foncé ; $2 b$, limon gris ; 3, limons à horizon "S» brun-rouge ; 4, limons et sol organo-minéral remaniés; 5 , blocage de pierre ; 6 , limons remaniés ; 7 , petit sol noir organique (gummifère); $8 a$, limons gris-verdâtre ; $8 b$, limons tufacés.

Gonesse (Val-d'Oise), La Fosse aux Larrons : west-east section (CP I) on the edge of bands 12 and 13 (altitudes in cm below the relative site level) with the position of samples taken for pollen (1 to 8, 10 and 13) and snails (9 to 12, sample 10 having been split in two):

1, tuff; 2a, grey silt with dark grey horizon; $2 b$, grey silt; 3, silts with brown-red ' $S$ ' horizon; 4, reworked silts and organic-mineral soil; 5, stone blocking; 6, reworked silts; 7, small black organic soil (gum-containing); 8a, grey-green silts; 8b, tufa silts.

de pierres calcaires blanches disposées en arc de cercle qui paraissait se poursuivre au nord en dessinant une couronne au diamètre interne de l'ordre de $8 \mathrm{~m}$.

Reprenant les sondages initiaux, la coupe ouest-est à la limite des bandes 12 et 13 (CP I, fig. 3 et fig. 15) permit d'établir la stratigraphie de l'ensemble conservé sur une cinquantaine de centimètres de hauteur qui fera plus loin l'objet d'une étude détaillée :

$1:$ tuf en place à surface irrégulière,

2 : limon gris d'épaisseur centimétrique variable - de l'ordre de $10 \mathrm{~cm}$ - comblant les dépressions de la surface du tuf,

3 : limon gris à inclusions brun-roux, 10 à $15 \mathrm{~cm}$,

4 : limon gris à inclusions noirâtres, environ $30 \mathrm{~cm}$,

5 : chape de pierres,

6 : limon formant une chape de terre de l'ordre d'un mètre d'épaisseur,

7 : mince niveau noir de quelques centimètres à nombreux débris végétaux carbonisés,

8a : limon gris verdâtre,

$8 \mathrm{~b}:$ tuf remanié,

Les vestiges archéologiques ont été rencontrés de la base de la couche 2 à la couche 6 (cf. infra). Soupçonnée dès l'origine, une inhumation centrale fut mise au jour à la surface de la couche 3, à une trentaine de centimètres sous le sol décapé, sans qu'un quelconque creusement de fosse ait pu être mis en évidence : elle était orientée sud-ouest / nord-est, tête vers l'ouest.

L'intérêt du site de La Fosse aux Larrons apparaissait ainsi multiple. Il s'agissait d'abord, malgré l'arasement dû aux décapages modernes, d'un site de plein air présentant plusieurs niveaux conservés en stratigraphie dont l'étude géologique fine, outre la compréhension de l'évolution de la plaine alluviale, devait permettre de bien situer la succession des occupations archéologiques. Celles-ci pouvaient en effet être au moins doubles, s'agissant d'une part d'un monument funéraire bien caractérisé et d'autre part de vestiges mobiliers diversifiés (céramique, lithique, faune) d'allure plus ancienne : on pouvait, mutatis mutandis et certes plus modestement, penser aux fameux dolmens à couloir sous tumulus de Dissignac à Saint-Nazaire en Loire-Atlantique et au « vieux sol» mésolithique que son implantation avait remanié. Ensuite, le type de sépulture mis au jour était jusqu'alors inconnu régionalement et au-delà. En effet, si l'on se réfère à l'importante recension réalisée quelques années plus tard sur le Nord de la France, le chapitre consacré aux manifestations funéraires (Blanchet, 1984, p.197-218), où Bronze ancien et moyen ne sont pas dissociés, mentionne de rares cas d'inhumations, essentiellement en position secondaire ou «satellite » et l'absence de couronne de pierres ailleurs qu'à Gonesse. En effet, l'incinération sous tumulus dont la pratique se généralisera au Bronze final, apparaît déjà comme majoritaire au moins dans ce secteur géographique, associée ou non à des urnes et à des cistes en pierres et le plus fréquemment circonscrite par un fossé simple ou double. Ainsi, même en l'absence de mobilier comme c'est le cas le plus fréquent pour les sépultures de cette période, l'analyse de la structure funéraire de Gonesse replacée dans son contexte paléo-environnemental, la possibilité de datations radiocarbone et l'étude d'un assemblage d'artefacts, vestiges d'une occupation antérieure, pouvaient-elles constituer des apports nouveaux pour des phases chronologiques encore fluctuantes.

\section{LES DATATIONS RADIOCARBONE}

Dans un premier temps, trois échantillons ont été adressés pour datation en décembre 1976 au centre des faibles radio-activités de Gif-sur-Yvette :

- le premier (95.277.6, B II c) comprenait les fragments de côtes et vertèbres de l'inhumé ;

- le second (95.277.6, B II a et b) était constitué de charbons de bois prélevés aux abords immédiats du squelette (couche 3); 


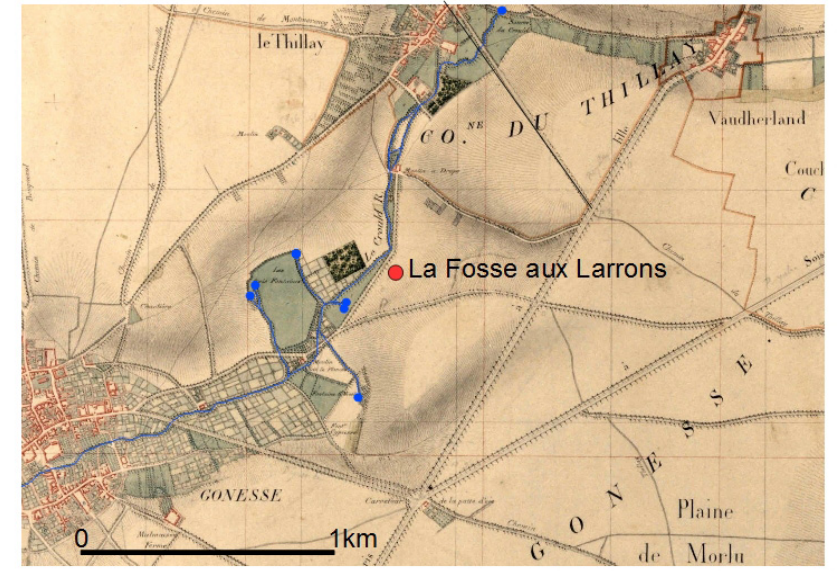

Fig. 4 - emplacement du site "La Fosse aux Larrons » dans la vallée du Crould (Feuille Etat Major 1819 n³0 Arrondissement de Pontoise, archives départementales du Val- d'Oise-SDAVO). Le site : • Le Crould : - et ses sources •

Location of the "Fosse aux Larrons" site in the Crould valley (Etat Major sheet $1819 n^{\circ} 30$ Arrondissement Pontoise, departmental archives of Val- d'Oise-SDAVO).

Le site: • The Crould: - and its sources •

- le troisième $(95.277 .6$, E) comprenait des matériaux carbonisés formant le sommet de la chape de terre extérieure au monument (couche 7).

Les résultats transmis en avril 1977 par G. Delibrias étaient respectivement les suivants :

- Gif - $4072: 3200 \pm 110$ ans

- Gif $-4073: 3580 \pm 200$ ans

- Gif - $4074: 3220 \pm 110$ ans

ce qui, en datations calibrées obligeamment transcrites par le centre de l'université de Lyon I en février 1999, puis révisées, donne :

- $\quad[1746,1135]$ avant J.-C. et avec un maximum de probabilités, 95,1\% [1746, 1208]

- $[2472,1454]$ avant J.-C. et avec un maximum de probabilités, 95,4\% [2472, 1488]

- $[1771,1213]$ avant J.-C. et avec un maximum de probabilités, 94,4\% [1771, 1252]

Le second échantillon, dont la faiblesse pondérale avait été signalée à l'envoi au laboratoire et qui aurait pu être complété, peut être considéré comme peu fiable s'agissant de charbons collectés épars dans l'épaisseur d'une couche, contrairement aux deux autres de nature homogène dont on constate d'ailleurs la bonne concordance malgré une composition distincte.

L'analyse de la faune a permis par la même occasion, comme il a été dit, de prélever un échantillon significatif dans les ossements de la couche 2 pour datation : le résultat transmis par le laboratoire de Lyon en février 1999 (LY - 9605) est de 4095 +/- 35 BP soit un âge calibré révisé récemment de 2866 à 2497 avant J.C. avec un maximum de probabilités, 71\% [2761, 2566].

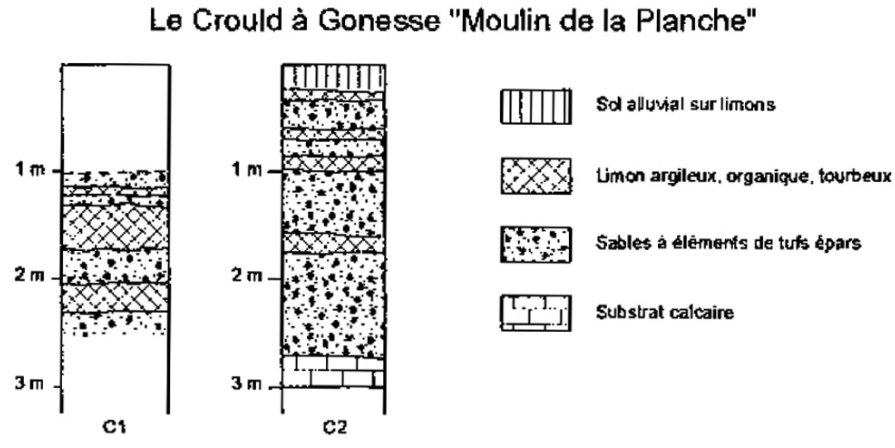

Fig. 5 - Coupes de Gonesse "le Moulin de la Planche»

(d'après P. Lebret, 1989).

Sections of Gonesse "le Moulin de la Planche"

(after P. Lebret, 1989).

\section{APPROCHE ENVIRONNEMENTALE}

\section{ANALYSE GÉOARCHÉOLOGIQUE}

\section{Contexte général}

Le site archéologique de Gonesse «La Fosse aux Larrons » (Brézillon, 1977) se situe en plein cœur de la vallée du Crould (fig. 4). Le Crould est une rivière de second ordre qui draine la Plaine de France à l'ouest du département du Val-d'Oise et rejoint la Seine en passant par Saint-Denis. La plaine alluviale est large d'environ $250 \mathrm{~m}$ au niveau du site. Les versants sont creusés dans les Calcaires de Saint-Ouen, les Sables de Beauchamp et les Calcaires du Lutétien.

\section{Contexte stratigraphiQue GéNÉRAL}

Les niveaux archéologiques sont installés dans des limons alluviaux reposant sur un niveau de tuf calcaire. Tuf et limons appartiennent aux formations de comblements alluviaux de la plaine d'inondation du Crould.

Plusieurs coupes stratigraphiques ont été relevées dans les formations alluviales du Crould (vallée dit du Rhin ou de la Daubée) lors d'interventions archéologiques plus récentes :

- coupes de Gonesse «Le Moulin de la Planche» (Lebret, 1989) (fig. 5),

- coupes du Crould à Goussainville et de La Daubée (Cecchini, 1990),

- coupe du Crould à Gonesse (Pastre et al., 1991) (fig. 6),

- coupes à Louvres «Le Bois d'Orville» (Bahain, 1997).

Ces relevés permettent de dresser un bilan stratigraphique du dernier comblement alluvial du Crould. D'amont en aval du site, le comblement sédimentaire varie de $9 \mathrm{~m}$ (La Daubée) à $16 \mathrm{~m}$ d'épaisseur (Louvres au «Bois d'Orville »). Le fond rocheux (bed-rock) est creusé dans les Calcaires du Lutétien et atteint les $51 \mathrm{~m}$ NGF à Louvres.

Les formations de comblement de la vallée peuvent être regroupées en trois grandes unités sédimentaires. 


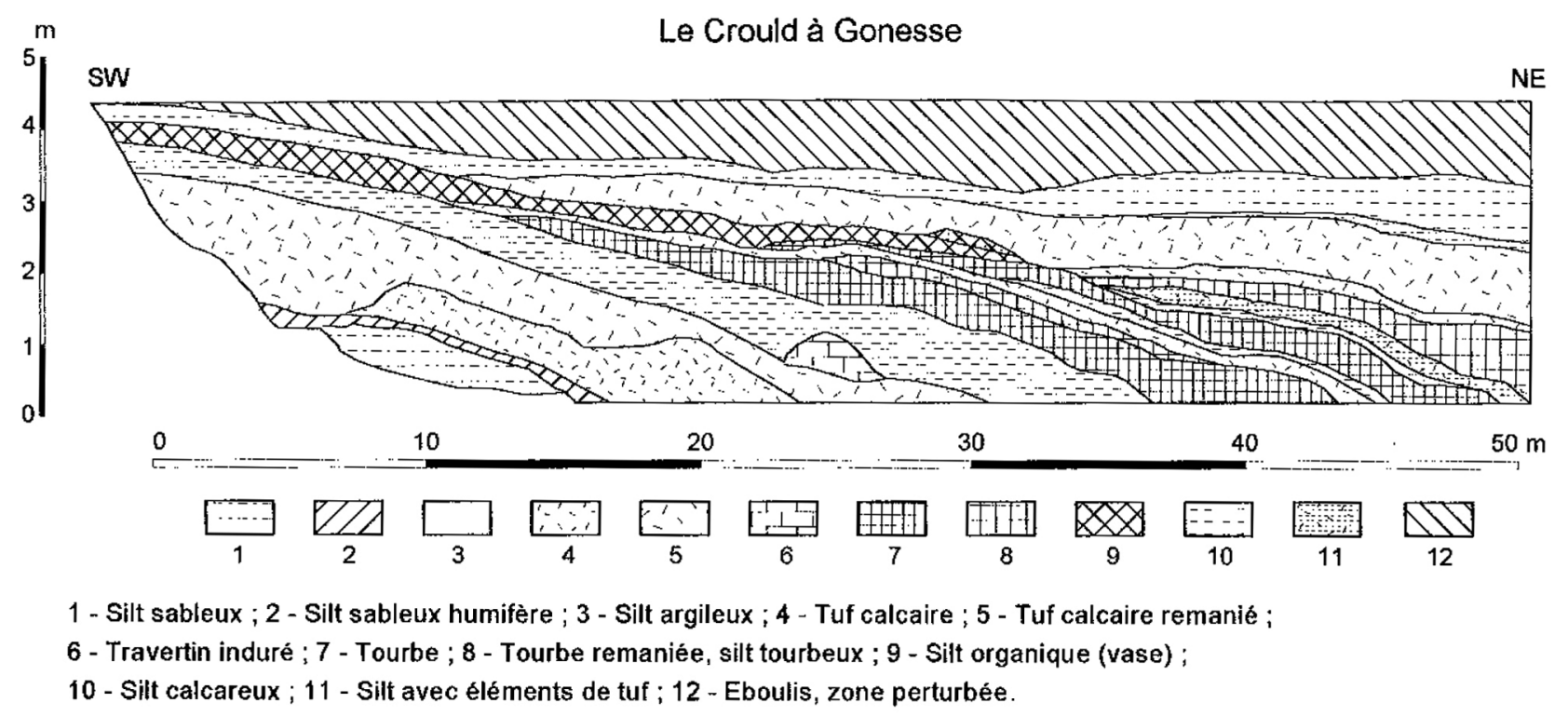

Fig. 6 - Coupe du Crould à Gonesse (d'après J.-F. Pastre et al., 1991).

Section of the Crould at Gonesse (after J.-F. Pastre et al., 1991).

\section{Les graviers, sables, limons et débris calcaires de base}

A la base, une unité alluviale reposant directement sur le substratum géologique est constituée de graviers, sables et limons accompagnés de débris calcaires sur les bords de versant. Constituée d'apports de versant et de dépôts alluviaux plus ou moins grossiers, elle présente une épaisseur de 1 à $3 \mathrm{~m}$ au plus. Par endroit, des reprises de creusement dans les formations grossières sont comblées par des limons argileux d'une épaisseur équivalente. Cette unité est attribuée au Tardiglaciaire (Cecchini, 1990).

\section{Les dépôts organiques, tourbes, limons et tuf}

Recouvrant la précédente, une unité de 7 à 4 m d'épaisseur est essentiellement constituée de dépôts organiques, tourbeux à passées limoneuses et de niveaux de tuf. Elle est la plus importante en épaisseur. Si les niveaux de tuf ne sont que sous la forme de traces sur les coupes en amont du site, à Gonesse par contre, les coupes (fig. 6) relevées par J.-F. Pastre (Pastre et al., 1991) et celles relevées par P. Lebret (Lebret, 1989) montrent dans la partie supérieure de cette seconde unité des niveaux de tuf épais en place ou remaniés (fig. 5) alternant avec des niveaux de limon argileux organique, tourbeux. Une date ${ }^{14} \mathrm{C}$ de $6510 \pm 80 \mathrm{BP}$ a été obtenue dans un des niveaux tourbeux de Gonesse (Bahain, 1997). Cette unité est attribuée au Boréal et à l'Atlantique.

\section{Les limons, sable-limoneux et limons argileux}

La dernière unité est composée de limons, sable-limoneux et limons argileux alluviaux ou colluviaux. Elle présente entre 2 et $4 \mathrm{~m}$ au maximum d'épaisseur. Elle est attribuée au SubBoréal et début du Sub-Atlantique (fig. 5). A Louvres, au
« Bois d'Orville », un habitat du Haut Moyen Âge a été fouillé dans la partie supérieure de ces niveaux (Bahain, 1997).

Les coupes du «Moulin de la Planche » à Gonesse (fig. 5) ont été pratiquement relevées sur les mêmes lieux que le site archéologique, là où «deux décharges s'élèvent de part et d'autre à plus de $30 \mathrm{~m}$ au-dessus de la vallée» (Lebret, 1989). La plus complète présente un remplissage de près de $3 \mathrm{~m}$ d'épaisseur au-dessus du bed-rock du Lutétien. Ce remplissage est constitué pour l'essentiel de sable à éléments de tuf épars, entrecoupé de petits niveaux de limons argileux organiques à tourbeux, recouvert dans la partie supérieure d'un sol de surface sur limon alluvial de $20 \mathrm{~cm}$ d'épaisseur.

\section{Stratigraphie du site « La Fosse aux Larrons » : COUPE OUest-Est CP I, cOUPE Est-OUeSt CP II ET COUPE NORD-SUd CP III}

Les coupes CP I (fig. 3) et CP III (fig. 8 et fig. 15) constituent des relevés stratigraphiques transversaux du site archéologique avec son tumulus d'ouest en est et du nord au sud. L'intervention archéologique a permis d'atteindre, $60 \mathrm{~cm}$ sous la surface de décapage et sous les remblais actuels, un tuf calcaire sur lequel reposent les niveaux stratigraphiques contenant les deux niveaux d'occupation du site.

\section{Le tuf (1) ${ }^{1}$ (prélèvement $\underline{4}$ )}

Un niveau de tuf homogène (1) constitue la base de la stratigraphie du site archéologique, $60 \mathrm{~cm}$ sous la surface

1. Les niveaux stratigraphiques sont numérotés d'un chiffre et les évènements (creusement, arasement, niveau d'occupation, etc. ...) le sont de lettres majuscules. 


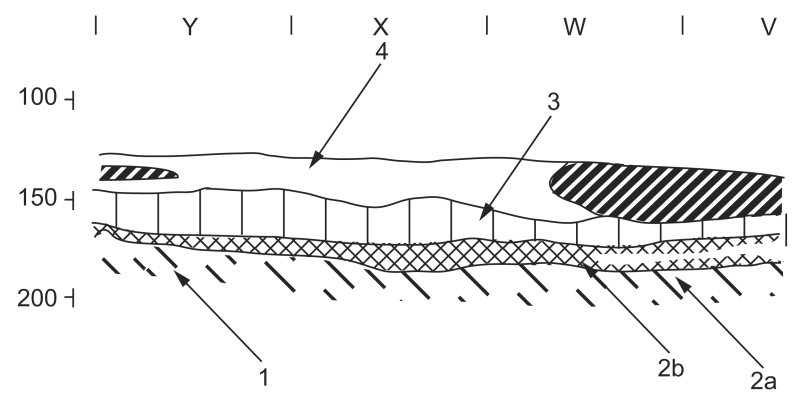

Fig. 7 - Coupe Est-Ouest CP II (cotes altimétriques en cm sous le niveau relatif de chantier) : 1, tuf ; 2a, limon gris à horizon gris foncé ; $2 b$, limon gris ; 3 , limons à horizon « $S$ » brun-rouge; 4 , limons et sol organo-minéral remaniés.

East-west section CP II (altitudes in $\mathrm{cm}$ below the site relative level): 1, tuff; 2a, grey silt with dark grey horizon; $2 b$, grey silt; 3 , silts with brown-red ' $S$ ' horizon; 4, reworked silts and organic-mineral soil.

de décapage. Il est calcaire, sableux, jaune et assez compact. Il présente une surface très irrégulière, avec des creux pouvant atteindre $15 \mathrm{~cm}$ d'amplitude. La topographie du toit du tuf $(\mathrm{CP}$ III fig. 8) montre que celui-ci plonge du sud-est vers le nordouest selon les altitudes relatives du chantier : de $-155 \mathrm{~cm}$ à -190 $\mathrm{cm}$.

Ce tuf appartient aux mêmes formations que celles observées dans la partie supérieure des coupes (fig. 5) relevées à Gonesse $\mathrm{au}$ « Moulin de la Planche» (Lebret, 1989).

\section{Les limons gris (2a - 2b) (prélèvement 10) et premier niveau archéologique (A)}

Les involutions du tuf sont comblées de 15 à $20 \mathrm{~cm}$ de limon gris (2b) présentant un horizon gris foncé à la base (2a). Ces limons gris correspondent à une reprise de l'alluvionnement. Cette formation n'a été préservée que dans les parties basses du tuf vers le nord-ouest et dans les creux du tuf. L'horizon gris foncé (2a) contient un niveau d'occupation archéologique (A) préservé dans les creux et la partie basse du tuf (CP III fig. 8). La répartition et la densité du matériel archéologique correspondent au même critère de niveau que ceux des limons gris. Ce niveau d'occupation (A) est antérieur à l'installation du tumulus.

\section{La première phase de remaniement (B) et niveau de matériel archéologique épars (C).}

Le toit des limons gris ne dépasse que rarement la côte de $-175 \mathrm{~cm}$ d'altitude relative du site. Il semble qu'une phase d'arasement ait repris le sommet du tuf sous-jacent dans les parties hautes. Cette surface correspond à une phase de remaniement (B) qui est souligné par un niveau de matériel archéologique (C) repris probablement de la couche archéologique sousjacente (A). Le niveau archéologique (A) a probablement été remanié et étalé à l'occasion de pratiques agricoles postérieures, à l'origine de l'horizon brun-rouge (3) suivant.

\section{Le limon à horizon " $\mathrm{S}$ » brun-rouge (3) (prélèvement $\underline{8}$ )}

Ce niveau est constitué d'un limon sur lequel s'est développé un horizon un peu plus dense (argileux) de 10 à $20 \mathrm{~cm}$ d'épaisseur présentant une couleur foncée brun à inclusions rouges. Ce niveau semble constitutif d'un horizon structuré « $\mathrm{S}$ » marquant ainsi une certaine stabilité permettant le développement d'un sol brunifié sur limons alluviaux (Fluviosol brunifié) : arrêt de l'accumulation au profit de la pédogenèse. La présence d'inclusions rouges dans cet horizon et la surface d'arasement sous-jacente (B) pourraient correspondre à une perturbation de l'horizon par des pratiques agricoles anciennes (Horizon labouré « $\mathrm{L} »)$ : labours ou défrichements récurrents. L'absence d'involution dans les parties les plus hautes du tuf et l'arasement du niveau gris sous-jacent concorderaient avec cette hypothèse (CP III, fig. 8). Il manque à cet horizon brunifié et perturbé (« $\mathrm{S}$ » et « $\mathrm{L} »)$ son horizon de surface organo-minéral « $\mathrm{A} »$.

\section{La surface d'arasement $(\mathrm{D})$ et la sépulture (E) (prélève- ment 13)}

La coupe CP I Ouest-Est en C-D-E (CP I fig. 3) montre assez clairement que l'installation du tumulus a été précédée par un creusement important jusqu'au tuf, $3 \mathrm{~m}$ en périphérie de la sépulture pour atteindre la cote de $-175 \mathrm{~cm}$ là où le tuf est le plus en surface (partie est du tumulus). A l'intérieur du périmètre défini par ce creusement, l'horizon de surface organo-minéral «A » du Fluviosol brunifié semble avoir été éliminé. C'est sur cette surface d'arasement (D) que la sépulture (E) a été installée au sommet de l'horizon brun-rouge (3).

\section{Les remblais archéologiques : les limons et le sol organo-minéral remaniés (4), blocage de pierre (5) (prélèvement $\underline{7}$ ) et les limons remaniés (6) (prélève- ments $\underline{3}$ et $\underline{6}$ )}

Les terres extraites suite à l'arasement et au creusement (D) préalable à la sépulture (E) ont pu servir à la mise en place du noyau de terre du tumulus. Les «paquets » de sédiments limoneux noirs organiques (CP II et CP III, fig. 7 et 8) mélangés aux limons (4) témoignent du remaniement opéré et de la présence d'un horizon organo-minéral « $\mathrm{A}$ » perturbé par les travaux d'aménagement. Il s'agit probablement de l'horizon organo-minéral de l'horizon brun-rouge (3) du Fluviosol brunifié (« $\mathrm{S}$ » et « $\mathrm{L} »)$ qui aurait été systématiquement extrait sous le tumulus.

Un blocage de pierres (5) recouvre ce premier remblaiement. Il prend son assise dans le creusement opéré en périphérie de la sépulture. Sa composition, calcaire, grès et silex, laisse à penser que ces blocs ont été récupérés sur les versants de la vallée.

Ce niveau de pierres (5) est lui-même recouvert d'un second remblai de limons remaniés (6). Cet ensemble de remblais avec la sépulture constitue le second ensemble archéologique du site.

\section{Le petit sol noir organique (gummifère) (7) (prélève- ment $\underline{5}$ )}

En surface de ce second remblai de limon (6) est installé un petit sol limoneux noir organique (7), de 3 à $4 \mathrm{~cm}$ d'épaisseur (CP I et CP III, fig. 3 et 8). Il n'est pas impossible que ce niveau organique soit constitué d'éléments de l'horizon organo- 


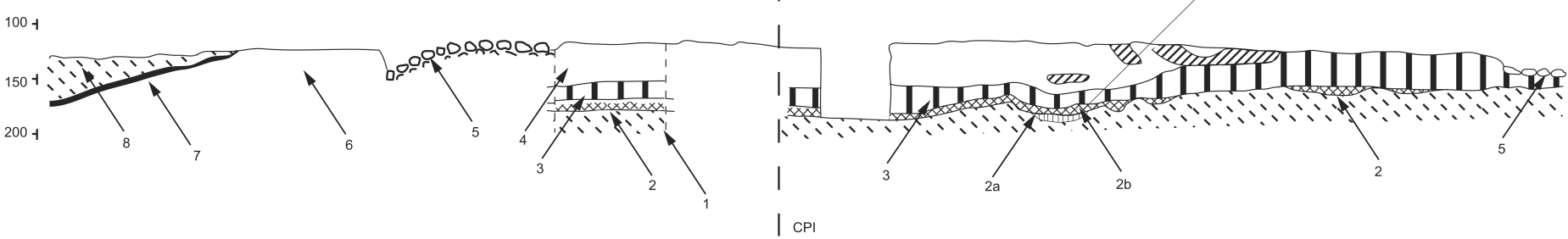

Fig. 8 - Coupe synthétique Nord-Sud CP III (cotes altimétriques en cm sous le niveau relatif de chantier) : 1, tuf; 2a, limon gris à horizon gris foncé ; $2 b$, limon gris ; 3 , limons à horizon «S» brun-rouge ; 4 , limons et sol organo-minéral remaniés ; 5 , blocage de pierre ; 6 , limons remaniés; 7 , petit sol noir organique (gummifère); $8 a$, limons gris-verdâtre ; $8 b$, limons tufacés.

General North-South section CP III (altitudes in $\mathrm{cm}$ below the site relative level): 1, tuff; 2a, grey silt with dark grey horizon; $2 b$, grey silt; 3, silts with brown-red 'S' horizon; 4, reworked silts and organic-mineral soil; 5, stone blocking; 6, reworked silts; 7, small black organic soil (gum-containing); $8 a$, grey-green silts; 8 b, tufa silts.

minéral «A» remanié et rapporté sur la surface du tumulus pour « gazonnage ». Il se peut aussi que ce petit sol organique marque un horizon gummifère « $\mathrm{H} »$ formé en milieu saturé d'eau en continuité avec la reprise d'une sédimentation alluviale marquée par les limons gris-verdâtre suivants.

\section{Les limons gris-verdâtre (8a) (prélèvement $\underline{2}$ ) et tufacés $(8 \mathrm{~b})$ (prélèvement 1 )}

Les niveaux suivants, les limons gris-verdâtre en premier (8a) puis les limons tufacés et tufs remaniés (8b) en second (CP I, fig. 3) recouvrent au fur et à mesure le tumulus sur plus de 30 $\mathrm{cm}$ d'épaisseur. Ces niveaux gris-verdâtre marquent une nette hydromorphie du site. La sédimentation limoneuse correspond à une reprise des apports alluviaux et la présence de tuf à un milieu aquatique d'eau courante.

\section{Remblai actuel (9)}

Le site est finalement recouvert sur 20 à $30 \mathrm{~cm}$ d'un remblai actuel.

\section{Commentaires}

Cette série sédimentaire et archéologique révèle une évolution des conditions de sédimentation et de pédogenèse dans laquelle s'imbriquent deux occupations archéologiques.

L'accumulation de tuf calcaire (1) est révélatrice d'un milieu très humide en eau permanente et courante, riche en végétaux proche des exutoires des sources proches du site (fig. 4). Un changement dans les conditions hydriques (abaissement du niveau d'eau) permet une occupation archéologique (A) en surface de ce niveau de tuf dans un contexte d'alternance entre alluvionnement et phase d'humidification favorisant le développement d'un petit horizon gris foncé (2a). Cet alluvionnement se poursuit (2b) en comblant les dépressions naturelles du tuf jusqu'à le recouvrir complètement (3). Une phase importante d'arrêt des alluvionnements limoneux permet le développement d'une pédogenèse de sol légèrement brunifié, structuré « $\mathrm{S}$ » caractéristique de sol alluvial sous couvert forestier. L'aspect brun avec des inclusions rouges et la présence d'une surface d'arasement laisse penser que ce sol a été perturbé par des pratiques agricoles « $\mathrm{L} »$ (labours / arrachements récurrents) qui ont arasé la surface du tuf dans sa partie la plus haute (autour de $-170 \mathrm{~cm}$ d'altitude relative du site). Seules les parties les plus profondes du tuf n'ont pas été touchées et c'est là où le premier niveau d'occupation (A) a pu être préservé dans les limons gris foncé (2a). Dans la partie du site où la surface du tuf est la plus haute, le matériel archéologique du niveau d'occupation a été étalé (C) à cette hauteur, sur le reste du site.

L'horizon organo-minéral « $\mathrm{A} » \mathrm{du}$ sol brunifié a entièrement disparu, soit par incorporation au sol au cours des pratiques agricoles, soit par son prélèvement systématique lors de l'aménagement du tumulus recouvrant la sépulture (E).

L'installation de la sépulture se fait directement sur les limons brun-rouge. Son recouvrement par des remblais issus du creusement des limons bruns révèle la présence effectivement de «paquets » d'un sol organique noir correspondant probablement à l'horizon « $\mathrm{A}$ » du sol Fluviosol brunifié perturbé par ces travaux d'aménagement.

Le tumulus installé, les alluvionnements reprennent sous la forme de limons gris-verdâtre (8a) déposés au pied du monument. En continuité avec ces limons gris-vert, un petit sol noir organique installé en surface du tumulus (7) révèle soit un «gazonnage » intentionnel de la surface du monument soit le développement d'une végétation (non arbustive) régulièrement inondée (6 mois dans l'année). Les dépôts alluviaux se poursuivent sous forme de limons gris avec du tuf soit remanié soit en place (8b) et révélateur alors d'un retour à un contexte beaucoup plus aquatique, en eau courante.

L'occupation du site, postérieurement au niveau (1) du tuf, semble se réaliser dans une période d'abaissement du niveau d'eau. Cet épisode postérieur à 5000 BP débute par le niveau d'occupation (A) dans un milieu encore très humide avec de fréquentes inondations. Il se poursuit dans un contexte forestier marqué par des pratiques agricoles entre 4000 et $3000 \mathrm{BP}$. Cette phase de couvert forestier se termine avec l'installation du tumulus qui sera soumis, dans une plaine alluviale de nouveau très humide, à des inondations fréquentes.

Les plaines alluviales sont des milieux extrêmement sensibles aux fluctuations des bilans hydriques des cours d'eau qui les drainent (Krier, 2004). Le site de « La Fosse aux Larrons », au cœur de la plaine du Crould, montre que la confrontation des observations pédo-sédimentaires, des 


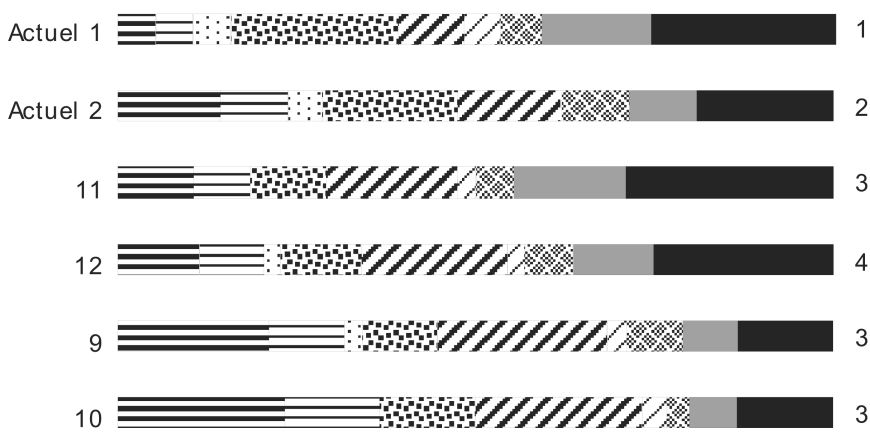

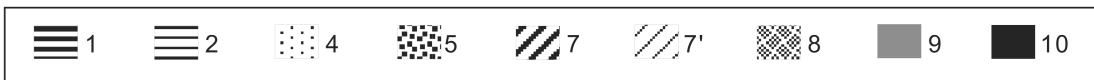

Fig. 9 - Gonesse (Val-d'Oise), La Fosse aux Larrons : spectres malacologiques. Pour chaque échantillon sont tracés un spectre des espèces et un spectre des individus qui rendent compte du pourcentage représenté par chacun des groupes écologiques présents dans l'échantillon: 1, Espèces exclusivement liées à la forêt ; 2, Espèces semi-forestières ; 4, Espèces steppiques ;

5, Espèces de stations découvertes ; 7, Espèces mésophiles ; 7', Limaces ; 8, Espèces aimant l'humidité ; 9, Espèces palustres ; 10, Espèces aquatiques. Lorsque les deux spectres d'un échantillon sont équilibrés, cela indique un milieu homogène et stable dans le temps. Le fait qu'ils marquent des différences sensibles indique la proximité d'un autre biotope ou un changement des conditions environnementales.

Gonesse (Val-d'Oise), La Fosse aux Larrons : snail spectra. For each sample a spectrum for species and a spectrum for individuals are calulated, showing the percentage represented by each ecological group present in the sample: 1 , Species exclusively linked to forest;

2, Semi-forest species; 4, Steppe species; 5, Open ground species; 7, Mesophile species; 7', Slugs ; 8, Moisture-loving species;

9, Marsh species; 10; Aquatic species. When the two spectra for a sample are evenly distributed, this indicates an environment that is uniform and stable in time. If the spectra show marked differences, this reflects the proximity of another biotope or a change in environmental conditions.

analyses paléoenvironnementales et des données archéologiques permet d'introduire ces fluctuations comme facteur dans les problématiques d'occupation des territoires, même sur une courte période allant de la fin de l'Atlantique récent et au début du Sub-Boréal.

(V. Krier)

\section{ANALYSE MALACOLOGIQUE}

\section{A - Prélèvements actuels}

La faune des deux prélèvements actuels correspond bien aux sites dans lesquels ils ont été effectués : champ cultivé (actuel 1), talus boisé (actuel 2). Dans ce dernier cas cependant, la très grande abondance de l'espèce Aegopinella nitidula (tabl. I) exagère le caractère forestier de l'association qui ne comporte en fait que trois espèces forestières proprement dite. Il faut noter la présence dans les deux assemblages d'un fonds commun de taxons aquatiques qui provient du tuf remanié ou des inondations occasionnelles. La même chose se retrouvera dans les prélèvements fossiles 9 et 10 .

\section{B - PrélÈVements fossiles}

Les prélèvements ont été placés dans l'ordre suivant : 11 $12-9-10$ c'est-à-dire du plus récent au plus ancien d'après la coupe (fig. 9). Il est très probable cependant que la faune 12 soit mélangée.

- Prélèvement 11 : le spectre des espèces est très diversifié et nombreux sont les groupes écologiques représentés. Le spectre des individus est presque entièrement occupé par les groupes palustre et aquatique. C'est le cas typique d'une malacofaune de marécage permanent, où les espèces terrestres, nombreuses mais mal représentées en quantité, ont été recueillies çà et là par les eaux de ruissellement ou lors de la montée des crues en saison humide.

- Prélèvement 12 : les mollusques aquatiques n’occupent ici que $59 \%$ de la population totale contre $88,50 \%$ dans l'échantillon précédent. On pourrait penser à une association de bordure de marécage ; mais, étant donné que ce niveau correspond au sommet de la chape du tumulus, il s'agit nécessairement de matériaux rapportés. Ont-ils été empruntés au tuf de base 1 ? Ont-ils été contaminés postérieurement par la faune du niveau 11 ? Il est difficile de le dire. On peut remarquer toutefois que les groupes forestier, semi-forestier et de terrain découvert sont relativement bien représentés. Si dans le calcul des pourcentages, on négligeait les coquilles palustres et aquatiques, on obtiendrait les résultats suivants :forestier $6 \%$; semi-forestier $25,50 \%$; terrain découvert et steppique $38 \%$, ce qui donne un assemblage de forêt très ouverte, ou mieux, de clairière de forêt.

- Prélèvements 10 et 9 : ces deux échantillons ont donné des faunes pratiquement identiques. Les spectres sont assez équilibrés. Il s'agit d'association de forêt peu dense et d'un paysage stable. Au moment de la construction du tumulus, la forêt occupait l'ensemble du site ; le niveau 2, probablement en place, constituait le sol immédiatement avant la construction du tumulus. Le niveau 3 correspond au niveau 2, mais à l'extérieur du tumulus ; il a seulement été rapporté pour la construction. Les coquilles palustres et aqua- 


\begin{tabular}{|c|c|c|c|c|c|c|c|c|}
\hline \multicolumn{9}{|c|}{ Gonesse, Tumulus de la Fosse aux larrons } \\
\hline Groupe & & & \begin{tabular}{|c|} 
Actuel \\
1
\end{tabular} & \begin{tabular}{|c|} 
Actuel \\
2
\end{tabular} & 11 & 12 & 9 & 10 \\
\hline \multirow[t]{8}{*}{1} & Acanthinula & aculeata & & & 2 & 3 & 37 & 9 \\
\hline & Aegopinella & nitidula & & 422 & 4 & 13 & 120 & 35 \\
\hline & Aegopinella & pura & & & 6 & 4 & 73 & 13 \\
\hline & Clausilia & bidentata & 2 & 9 & 3 & 3 & 20 & 5 \\
\hline & Cochlodina & laminata & & & & & 6 & 3 \\
\hline & Helicodonta & obvoluta & & & & 1 & 7 & 3 \\
\hline & Macrogastra & rolphii & & & & & 3 & 2 \\
\hline & Merdigera & obscura & & 5 & & & 2 & \\
\hline \multirow[t]{4}{*}{2} & Cepaea & sp. & 4 & 40 & 2 & 8 & 40 & 10 \\
\hline & Discus & rotundatus & & 132 & 22 & 62 & 190 & 70 \\
\hline & Pomatias & elegans & & & & 20 & 90 & 18 \\
\hline & Vitrea & crystallina & & & 3 & 8 & 89 & 18 \\
\hline 4 & Helicella & sp. & 22 & 5 & & 3 & & 1 \\
\hline \multirow[t]{5}{*}{5} & Vertigo & pygmaea & 2 & 7 & 1 & 1 & 4 & 3 \\
\hline & Pupilla & muscorum & 41 & 16 & 3 & 30 & 37 & 13 \\
\hline & Vallonia & costata & 17 & 107 & 2 & 37 & 31 & 29 \\
\hline & Vallonia & pulchella & 52 & 17 & 24 & 50 & 33 & 17 \\
\hline & Truncatellina & cylindrica & & & & 17 & & \\
\hline \multirow[t]{10}{*}{7} & Cochlicopa & lubrica & & 5 & 7 & 18 & 28 & 12 \\
\hline & Clausilia & rugosa & & & & 5 & 10 & 2 \\
\hline & Euconulus & fulvus & & & 2 & 1 & & \\
\hline & Helicigona & lapicida & & & & & 4 & 1 \\
\hline & Nesovitrea & hammonis & 1 & & 6 & 4 & 3 & 3 \\
\hline & Oxychilus & sp. & & & 1 & 9 & 7 & \\
\hline & Punctum & pygmaeum & & & 3 & 4 & 1 & \\
\hline & Trochulus & hispidus & 45 & 70 & 9 & 27 & 110 & 33 \\
\hline & Vitrea & contracta & & & 4 & 4 & 20 & 17 \\
\hline & Vitrina & pellucida & & 1 & & 1 & 2 & 3 \\
\hline $7^{\prime}$ & Limax & sp. & 3 & & 3 & 1 & 2 & 2 \\
\hline \multirow[t]{3}{*}{8} & Carychium & tridentatum & & 18 & 12 & 29 & 234 & 66 \\
\hline & Succinella & oblonga & 4 & 3 & & 2 & 2 & \\
\hline & Vertigo & angustior & & & 9 & 11 & 1 & \\
\hline \multirow[t]{6}{*}{9} & Carychium & minimum & & & 18 & 1 & & \\
\hline & Succinea & putris & 3 & 3 & 39 & 11 & 8 & 2 \\
\hline & Vertigo & antivertigo & 2 & 1 & 19 & 4 & 1 & 3 \\
\hline & Vertigo & moulinsiana & & & 1 & & & \\
\hline & Vallonia & enniensis & & & 1 & 7 & 3 & \\
\hline & Zonitoides & nitidus & 1 & & 2 & 2 & & \\
\hline \multirow[t]{13}{*}{10} & Valvata & cristata & 2 & 3 & 613 & 465 & 5 & 3 \\
\hline & Valvata & piscinalis & & 3 & & 24 & & \\
\hline & Bithynia & tentaculata & 5 & 2 & 229 & 19 & 26 & 13 \\
\hline & Galba & truncatula & 2 & & 24 & 3 & 1 & \\
\hline & Radix & labiata/balthica & & & 130 & 1 & 3 & 3 \\
\hline & Lartetia & sp. & & & & & 1 & \\
\hline & Limnaea & sp. & 2 & & 10 & 1 & & \\
\hline & Planorbis & carinatus & 1 & 1 & 102 & 6 & & \\
\hline & Gyraulus & albus & & & 38 & 8 & & \\
\hline & Gyraulus & crista & & & 107 & 58 & & \\
\hline & Bathyomphalus & contortus & & & 41 & 1 & & \\
\hline & Hippeutis & complanatus & & & 5 & & & \\
\hline & Pisidium & sp. & & & 303 & 4 & & 1 \\
\hline \multicolumn{3}{|c|}{ Somme des individus } & 211 & 870 & 1810 & 991 & 1254 & 413 \\
\hline \multicolumn{3}{|c|}{ Somme des espèces } & 19 & 21 & 38 & 44 & 37 & 31 \\
\hline & Cecilioides & acicula & 5 & 5 & & 9 & 7 & \\
\hline
\end{tabular}


4 Tabl. I - Gonesse (Val-d'Oise), La Fosse aux Larrons : liste des malacofaunes collectées. L'espèce Ceciliodes acicula est un mollusque fouisseur de mœurs souterraines, il n'est donc pas contemporain des niveaux dans lesquels il est récolté et n'a pas été pris en compte lors des comptages, sa fréquence d'apparition apparaît donc en fin de tableau. Groupes écologiques : voir la légende de la fig. 10.

Gonesse (Val-d'Oise), La Fosse aux Larrons : list of snail fauna recovered. The species Ceciliodes acicula is a mollusc that burrows underground and is thus not contemporary with the layers in which it was found. It is not included in the counts and its frequency is given at the end of the table. Ecological groups: see caption for fig. 10.

tiques en petit nombre sont, à l'instar de celles des assemblages actuels, soit remaniées du tuf 1 , soit apportées par des inondations ponctuelles pendant la formation du sol 2 .

En ce qui concerne l'âge, on ne peut rien avancer de précis. Il est probable cependant que le tuf du niveau 8 a (échantillon 11) correspond à la phase climatique très humide du début du Subatlantique (vers 2400 B.P.). Quant au tuf ancien, niveau 1, il pourrait correspondre à une autre phase très humide au début de l'Atlantique (vers 7500 B.P.). Entre ces deux phases, le climat était plus sec, particulièrement à la fin de l'Atlantique et au début du Subboréal (entre 5000 et 4000 B.P.) (Puisségur, 1976). Le fait qu'en 9 et 10 on ne trouve pas Vertigo alpestris semble exclure pour le sol 2 un âge antérieur à 5000 B.P. (Puisségur, 1976 ; Limondin, 1992).

\section{(J.-J. Puisségur \& N. Limondin-Lozouet)}

\section{ANALYSE PALYNOLOGIQUE}

L'analyse pollinique du tumulus de Gonesse « La Fosse aux Larrons » a été réalisée par Gérard Firmin durant l'hiver 1976/77, suite à la campagne de fouille menée en 1976 par Michel Brézillon et Jacques Tarrête (Brézillon, 1977). L'étude et son interprétation (Firmin, 1977) ont été menées selon les protocoles en vigueur durant les années soixante-dix avec déjà une approche méthodologique novatrice : attention portée à l'état de conservation des stocks polliniques et à la nature des microrestes non polliniques présents sur les lames. Cependant, la démarche palynologique, appliquée aux sédiments archéologiques, a considérablement évolué depuis trente ans. En effet, une attention particulière est aujourd'hui portée aux conditions de la sédimentation pollinique (état de conservation du matériel, concentration en stocks polliniques, diversité taxinomique et nature du palynofaciès ${ }^{2}$ ) afin de définir le niveau de fiabilité des enregistrements et leur représentativité. De ce fait, la publication des analyses réalisées par G. Firmin est un exercice délicat qui consiste à restituer les travaux effectués, il y a plus de trente ans, tout en les interprétant en fonction des avancées méthodologiques développées depuis. Aussi, après un rapide exposé sur l'actualisation des méthodes, le présent article va reprendre les données acquises en 1977 puis en proposer une lecture et une interprétation qui diffèrent sensiblement de celles initialement avancées par l'auteur. Enfin, ces résultats, obtenus dans une structure archéologique, seront comparés avec les enregistrements de séquences contemporaines mais issues de paléochenaux à remplissage organique.

2. Le palynofaciès est l'ensemble des éléments présents sur les lames de préparations microscopiques : micro-débris végétaux, matière organique amorphe, micro-charbons, pollens, spores d'algues et de moisissures...

\section{QUELQUES PRINCIPES MÉTHODOLOGIQUES SUR L'INTERPRÉTA-} TION DES DONNÉES POLLINIQUES

L'interprétation des données polliniques repose sur différents critères. Les caractères écologiques des taxons en présence (Bournerias, 1979 ; Rameau et al., 1989) permettent l'appréciation des contextes environnementaux tandis que les activités humaines sont appréhendées par les marqueurs d'anthropisation et l'ouverture plus ou moins prononcée du milieu (Aaby, 1986 ; Behre, 1981 ; Jalut, 1991 ; Richard, 1995 ; Leroyer, 1997 ; Brun, 2007). L’interprétation biostratigraphique des différents niveaux se réfère aux grandes étapes de l'histoire de la végétation régionale, soit celle établie pour le Bassin parisien dans le cas de Gonesse (Leroyer, 1997). Ces différentes interprétations font également référence aux acquis sur la dissémination pollinique puisque les pollens ne sont pas produits en quantité équivalente et que leurs agents de transport diffèrent; il en résulte une sous- ou sur-représentation des différents taxons et des apports plus ou moins lointains (Heim, 1970 ; Barthélémy 1976 et 1985 ; Triat-Laval, 1978 ; Gaillard et al., 2008). Outre ces facteurs qui impliquent qu'un spectre pollinique n'est en rien un spectre de la végétation, le milieu d'échantillonnage influence également la perception de l'environnement végétal. Ainsi, l'étude palynologique d'un tumulus autorise difficilement, seule, une approche environnementale ; elle doit être confortée par des référentiels acquis dans des milieux plus propices à la discipline. Cette restriction tient au contexte de l'échantillonnage en structures archéologiques et à la nature des sédiments traités, rarement organiques et humides, parfois repris (Leroyer, 2006a). En effet, une structure est souvent de taille restreinte et la pluie pollinique n'y est pas piégée comme en contexte naturel ; la pollinisation de la végétation locale y est majorée par rapport aux apports polliniques du voisinage. Certaines formations végétales peuvent, de surcroît, être favorisées par des rejets humains (Richard, 1985 ; Bouchet et al., 1992 ; Leroyer, 1994). De plus, le colmatage de la structure peut relever d'un remblaiement rapide et anthropique ; dans ce cas, les stocks polliniques rencontrés lors de l'analyse n'illustrent pas l'environnement végétal de la structure mais reflètent le contenu en pollens résiduels des sédiments volontairement apportés qui peuvent être d'âge bien antérieur. Enfin, le comblement doit être propice à la conservation des stocks polliniques, donc le plus organique et humide possible (Reille, 1990). De ce fait, une des principales limites aux analyses de structures archéologiques réside dans l'altération du matériel car tous les types polliniques n'ont pas la même résistance à la corrosion. Il en découle une distorsion importante des enregistrements puisque les grains les plus fragiles sont rapidement détruits, entraînant un « enrichissement » artificiel en taxons résistants (Havinga, 1984).

Pour vérifier la représentativité des enregistrements, des protocoles d'évaluation de la fiabilité des données ont progressivement été mis en place (Reille, 1990 ; Leroyer, 1997 et 
Tabl. II - Gonesse (Val-d'Oise), La Fosse aux Larrons : données sur la sédimentation pollinique.

Gonesse (Val-d'Oise), La Fosse aux Larrons: data on pollen sedimentation.

\begin{tabular}{|c|c|c|c|c|c|c|}
\hline Échantillon & Sédiment & $\begin{array}{l}\text { Nombre de pollens } \\
\text { et de spores comptés }\end{array}$ & $\begin{array}{c}\text { Diversité } \\
\text { taxinomique }\end{array}$ & $\begin{array}{c}\text { État de } \\
\text { conservation }\end{array}$ & $\begin{array}{c}\% \text { de } \\
\text { Cichorioïdées }\end{array}$ & Palynofaciès \\
\hline 1 & limons & 272 & 22 & $\mathrm{nr}$ & 16 & $\mathrm{nr}$ \\
\hline 2 & limons & 238 & 20 & $\mathrm{nr}$ & 41 & $\mathrm{nr}$ \\
\hline 3 & limon brun-gris & 229 & 14 & très mauvais & 86 & $\mathrm{nr}$ \\
\hline 6 & limons remaniés & 164 & 17 & très mauvais & 64 & D.O, Mc. \\
\hline 7 & limons remaniés & 278 & 17 & $\mathrm{nr}$ & 53 & D.O, Mc. \\
\hline 13 & limon sous le crâne & 349 & 25 & $\mathrm{nr}$ & 14 & $\mathrm{nr}$ \\
\hline 4 & tuf alluvial & 264 & 20 & $\mathrm{nr}$ & 36 & $\mathrm{nr}$ \\
\hline
\end{tabular}

\author{
$\mathrm{nr}=$ non renseigné \\ D.O = débris opaques \\ Mc. $=$ microcharbons \\ M.O.A $=$ matière organique amorphe
}

2006a). Ils tiennent compte de la concentration en stocks polliniques, de l'état de conservation du matériel, de la diversité taxinomique, de l'équilibre des spectres et de la nature du palynofaciès. De plus, les décomptes gagnent à être poussés jusqu'à l'obtention de sommes supérieures à 300 grains tandis que l'individualisation d'au moins 20 taxons est jugée nécessaire pour qu'un spectre pollinique puisse être représentatif d'un paysage végétal (Cushing, 1967 ; Reille, 1990 ; Leroyer, 1997). Ces démarches permettent de repérer les niveaux souffrant d'une quelconque distorsion liée à la sédimentation pollinique.

\section{Les DONNÉES OBTENUES en 1977}

\section{Nature et origine des prélèvements}

L'étude palynologique concerne dix échantillons, issus des principales unités sédimentaires qui participent à la stratigraphie du site (Krier, supra). Prélevés en différents points, ils s'organisent selon la succession suivante (fig. 3).

- Léchantillon 4 provient du tuf alluvial (c. 1), reconnu dans la plupart des coupes dégagées dans la vallée du Crould (Lebret, 1989 ; Pastre et al., 1991 ; Bahain, 1997). Datée de 6510+/-80 BP à Gonesse (Bahain, 1997), cette formation tufacée est rapportée à l'Atlantique.

- L'échantillon 10 a été prélevé dans le dépôt limoneux gris (c. 2) qui vient combler les dépressions de la surface irrégulière du tuf : il est également antérieur à l'installation du tumulus et a été daté de 4095 +/- 35 BP (Ly 9605).
- L'échantillon 8 est issu du niveau limoneux brun-roux (c. 3), pouvant constituer l'horizon illuvial d'un sol de surface (Krier, supra). Des charbons, prélevés dans cette unité, ont donné un âge de 3580 +/- 200 BP (Gif 4073).

- L'échantillon 13 a été prélevé sous le crâne (carré Z 15) et a été daté de 3200 +/- 110 BP (Gif 4072).

- L'échantillon 7 provient du noyau de sédiments présents sous la chape de pierre du tumulus (c. 4). Lors de l'aménagement de la structure, ces dépôts ont vraisemblablement été extraits du sol sous-jacent dont ne subsiste que l'horizon illuvial brun-roux (Krier, supra).

- L'échantillon 6 est issu de la chape de limons qui recouvre les pierres de la sépulture (c. 6).

- L'échantillon 3 provient également des remblais qui coiffent la sépulture mais il a été prélevé latéralement dans une formation limoneuse brun-gris (c. 6).

- L'échantillon 5 est issu du petit sol humifère noir, présent à la surface de la chape limoneuse qui couvre le tumulus (c. 7) ; il a été daté sur charbon de 3220 +/- 110 BP (Gif 4074).

- Léchantillon 2 a été prélevé dans les formations limoneuses gris-vert qui encerclent le tumulus et l'ont peu à peu recouvert (c. 8a).

- L'échantillon 1 provient de la partie supérieure des dépôts limoneux qui se sont mis en place après l'édification du tertre (c. 8b).

Au laboratoire, les préparations physico-chimiques ont été adaptées à la nature des échantillons (Firmin, 1977). Tous les 
sédiments ont subi une décalcification par $\mathrm{HCl}$, une élimination des composants siliceux par HF et une destruction de la matière organique par $\mathrm{KOH}$, mais les plus organiques $(1,3$ et 4) ont été soumis à une attaque au perborate de sodium. La méthode de Schültze a été appliquée aux prélèvements chargés en cendres (éch. 2, 5, 6, 7 et 15) tandis que les échantillons 8 et 10 ont fait l'objet d'une lévigation en liqueur lourde (Girard et Miskovsky, 1969).

\section{Appréciation de la sédimentation pollinique (tabl. II)}

Quelques informations sur la nature du palynofaciès nous sont parvenues puisque G. Firmin (1977) a consigné certaines remarques à ce sujet, démarche fort novatrice pour l'époque. A l'exception du niveau basal, tous les échantillons ont présenté une charge en débris opaques et en micro-charbons : celle-ci étant particulièrement élevée dans les prélèvements $10,8,15,7$, $6,5$ et 2 . Seuls quelques échantillons ( 4,8 et 2$)$ ont témoigné de la présence de matière organique amorphe.

L'évaluation de la concentration en stocks polliniques est plus difficile à mener puisque trois méthodes de préparations physico-chimiques ont été appliquées et que les approches en concentrations absolues (Stockmarr, 1972 ; Cour, 1974) n’étaient alors pas développées en palynologie archéologique. Une information nous est livrée par l'analyste qui dénonce la pauvreté de l'échantillon 10 pour lequel la lecture de 18 lames a été nécessaire pour n'obtenir qu'un total de 144 pollens et spores ; ce niveau peut être considéré comme sub-stérile. De même, l'échantillon 6 devait présenter de très faibles concentrations puisque le décompte est inférieur à 200 grains, soit la somme jugée alors représentative.

Seules quelques informations sont spécifiées au sujet de la conservation du matériel : elles concernent les prélèvements où les pollens étaient fortement corrodés (échantillons 10, 3 et 6) mais l'importance des grains indéterminables n'est pas renseignée. Des indications sur l'état de conservation des stocks polliniques nous sont, toutefois, indirectement fournies par l'abondance des Composées et tout particulièrement des Cichorioïdées, types polliniques particulièrement résistants qui se trouvent favorisés et « artificiellement » sur-représentés lorsqu'une forte altération du matériel détruit les grains les plus fragiles (Havinga, 1984). La précision, apportée par G. Firmin, sur la forte corrosion des pollens dans les échantillons 3 et 6 est confortée par l'importance des Cichorioïdées dans ces deux niveaux (fig. 12) mais elle est moins nette pour l'échantillon 10. En revanche, les prélèvements 4,7 et 2 attestent également des fréquences élevées de ce taxon qui laissent présager une altération assez forte du matériel. La conservation des stocks polliniques semble donc globalement assez médiocre, voire mauvaise dans l'ensemble des échantillons du tumulus de Gonesse.

La prise en compte de la diversité taxinomique informe également sur la conservation et la représentativité des enregistrements. Elle n'atteint le seuil des 20 taxons que dans la moitié des échantillons $(4,8,13,2$ et 1$)$ et s'avère particulièrement faible dans les niveaux où les Cichorioïdées sont les plus abondantes $(3,7$ et 6$)$. De façon générale, la diversité n'apparaît pas très élevée et une liste de seulement 38 taxons a pu être établie pour l'ensemble de l'analyse (fig. 12).

\section{Organisation des cortèges polliniques}

Les dix spectres polliniques présentent certains points communs : prédominance des herbacées, guidées par les Cichorioïdées ou les Poacées, ligneux le plus souvent dominés par le tilleul (Tilia) et le noisetier (Corylus). Néanmoins, une évolution des cortèges est perceptible ; elle renvoie à la distribution stratigraphique des échantillons et permet d'individualiser cinq zones polliniques (fig. 10).

Zone Gon a = échantillons 4 et 10 (c. 1 et 2)

Bien qu'issus de formations bien différentes (tuf et limon gris foncé), les échantillons 4 et 10 s'apparentent de par leurs occurrences assez élevées de fougères et tout particulièrement de spores monolètes. Les Cichorioïdées y dominent les Poacées mais ces dernières sont assez étendues. Des céréales sont présentes tandis que le cortège rudéral est bien développé : il est guidé par les plantains (Plantago) dont les valeurs sont notables. Les ligneux sont assez diversifiés avec la présence de chênes (Quercus), ormes (Ulmus) et frênes (Fraxinus) aux côtés des tilleuls et des noisetiers. Les pins (Pinus) et les aulnes (Alnus) sont également assez abondants. La subdivision interne relève de la progression des ligneux et des fougères trilètes aux dépens des Composées (Cichorioïdées et Astéracées) dans l'échantillon 10.

\section{Zone Gon $\mathrm{b}=$ échantillons 8 (c. 3)}

Le prélèvement 8 , provenant de l'horizon illuvial d'un sol, traduit une certaine continuité avec les niveaux sous-jacents. Il témoigne de la persistance des céréales et du cortège rudéral ; ce dernier est plus diversifié mais le plantain y est moins développé. Les Cichorioïdées conservent une légère prédominance devant les Poacées. En revanche, les fougères sont beaucoup moins étendues. De même, des modifications se marquent au sein des ligneux avec la disparition de l'essentiel des composants de la chênaie (chênes, ormes et frênes) ; seuls les tilleuls persistent. Les pins sont moins nombreux mais des bouleaux (Betula) apparaissent et le lierre (Hedera) s'accroît.

\section{Zone Gon c = échantillon 13}

Le sédiment présent sous le crâne s'individualise par une très forte représentation du lierre. Le tilleul régresse alors que le noisetier augmente légèrement. Si les céréales diminuent, le cortège rudéral, guidé par les Chénopodiacées, Polygonum et Plantago, progresse.

\section{Zone Gon d = échantillons 7, 6 et 3 (c. 4 et 6 )}

Les échantillons 7, 6 et 3, prélevés dans les remblais limoneux de la chape du tumulus, se caractérisent par un large essor des Cichorioïdées aux dépens des Poacées, du cortège rudéral et des céréales qui ont totalement disparu. Cette modification est particulièrement nette dans l'échantillon 3 , où les Composées représentent près de $90 \%$ des pollens décomptés. La strate arborée diminue également : les noisetiers régressent nettement tandis que les aulnes, pins et bouleaux se maintiennent en faibles fréquences. Le lierre reste présent mais en valeurs beaucoup plus basses que dans l'échantillon 9. Si les tilleuls conservent d'abord des occurrences notables, ils s'effondrent dans l'échantillon 3 alors que tous les autres ligneux disparaissent. 


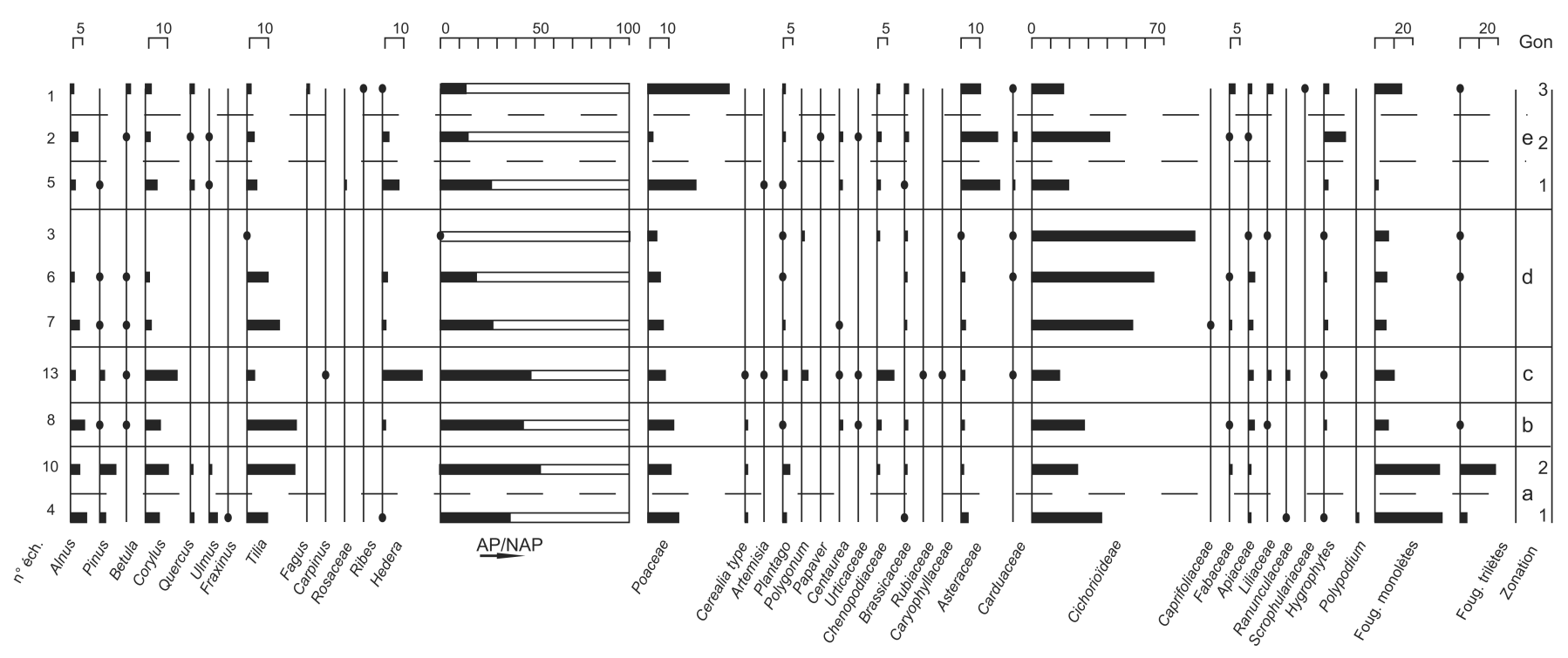

Fig. 10 - Gonesse (Val-d'Oise), La Fosse aux Larrons : diagramme pollinique (spores exclues de la somme de base / les échantillons dont le $n^{\circ}$ est encadré ont une somme de base inférieure à 200 pollens et spores).

Gonesse (Val-d'Oise), La Fosse aux Larrons: pollen diagram (grains excluded from the basic sum / samples whose number is framed have a basic sum less than 200 pollens and grains).

Zone Gon e = échantillons 5, 2 et 1 (c. 7 et 8 )

Les prélèvements 5,2 et 1 s'individualisent par le développement des Astéracées aux dépens des Cichorioïdées et par une diversification des ligneux. Si les céréales restent absentes, le cortège rudéral reprend quelque importance ; il est guidé par les plantains, les Centaurées (Centaurea), les Chénopodiacées et les Brassicassées. Des modifications des assemblages ont conduit à l'individualisation de trois sous-zones.

- Dans la première $(\mathrm{e} 1=$ éch. 5$)$, les Poacées connaissent une hausse notable. Les ligneux voient réapparaître Quercus et Ulmus aux côtés de Tilia. Corylus, Alnus et Hedera sont également enregistrés. Des Rosacées sont présentes.

- La seconde (e2 = éch. 2) témoigne de la chute des Poacées au profit des Cichorioïdées. Les hygrophytes (vraisemblablement des Cypéracées) culminent dans ce niveau. La plupart des ligneux (Hedera, Corylus, Quercus, Tilia) diminuent.

- La troisième $(\mathrm{e} 3$ = éch. 1) illustre une large reprise des Poacées aux dépens des Composées. Le cortège rudéral est moins diversifié. Les hygrophytes diminuent mais un cortège amphibie (Scrophulariacées, Liliacées, Fabacées, Apiacées) est présent. Les fougères, jusque là fort rares, augmentent. Certains ligneux (Alnus, Hedera, Tilia) diminuent encore, d'autres (Ulmus) disparaissent mais quelques-uns (Betula, Corylus, Quercus) progressent légèrement et Fagus (le hêtre) apparaît.

\section{INTERPRÉTATION DES DONNÉES}

La prise en compte des critères de la sédimentation pollinique conduit à considérer les données polliniques du site de Gonesse
« La Fosse aux Larrons » comme peu fiables. L'altération du matériel a induit une sur-représentation des Cichorioïdées, type pollinique le plus résistant, entrainant un déséquilibre des spectres. La corrosion des stocks polliniques est vraisemblablement aussi à l'origine de la faiblesse des concentrations et de la diversité, les grains les plus fragiles ayant été détruits. Ce biais taphonomique limite considérablement les possibilités interprétatives. Ainsi, l'approche environnementale et l'appréciation de l'anthropisation du milieu ne peuvent s'appuyer que sur l'aspect qualitatif, soit les caractères écologiques des taxons présents, en délaissant l'indice quantitatif puisque les fréquences illustrent principalement la résistance des types polliniques à la corrosion. Sur cette base, quelques modifications du paysage sont relatées par la séquence pollinique.

La première zone (éch. 4 et 10) témoigne d'un milieu encore assez boisé avec quelques pins et surtout la présence d'une chênaie-tillaie et d'une aulnaie à proximité de « La Fosse aux Larrons ». Pouvant participer à l'une ou à l'autre de ces formations, le noisetier indique que ces boisements sont assez clairs ou ont été pour partie défrichés, laissant place à des clairières. De même, la relative abondance des fougères relève probablement de leur développement sur les sols conquis sur l'aulnaie (Leroyer, 2003 et 2006b). Le paysage est, en effet, marqué par la présence humaine. L'importance des plantains au sein du cortège rudéral est significative de pratiques pastorales tandis que des cultures céréalières sont attestées. La différence entre les deux échantillons paraît liée à une hausse de la pression humaine : dans le limon gris, l'élevage semble se développer tandis que la chênaie comme l'aulnaie sont encore éclaircies, laissant la place aux essences de lumière et aux fougères. Daté de 4095 +/- 35 BP, ce niveau illustre le paysage du début du Subboréal et les activités des hommes de la fin du Néolithique. 
Le tuf basal pourrait dater de l'Atlantique récent mais le peu de variations perçues entre les deux spectres incline à le considérer comme sub-contemporain du limon sus-jacent ; hypothèse acceptable puisque la formation de tuf peut perdurer au début du Subboréal (Pastre et al, 2001, 2002, 2003).

La zone 2 (éch. 8) ne traduit que de très légères modifications environnementales. Des activités agro-pastorales sont toujours perçues mais la diversification du cortège rudéral évoque peut-être une modification des pratiques. La disparition des composants de la chênaie (autres que le tilleul) et le recul des pins peuvent être significatifs d'un défrichement de ces formations. Cette hypothèse se trouve renforcée par l'apparition des bouleaux aux côtés des noisetiers, tous deux étant des essences de lumière et de reconquête des zones déboisées. De même, la croissance du lierre tend à indiquer un éclaircissement accru des formations forestières.

Dans la zone 3 (éch. 13), l'abondance du lierre ne peut pas refléter sa seule pluie pollinique. Elle oriente vers une interprétation d'ordre cultuel et permet d'envisager un dépôt intentionnel de lierre («oreiller ») sous le crâne du défunt; de telles pratiques ont été mises en évidence dans des nécropoles protohistoriques (Leroyer, 1994). Cependant, une pollution postérieure, issue du sommet du tumulus ne peut être écartée. Elle s'avère invérifiable en l'absence d'informations précises sur l'état de conservation des pollens de lierre par rapport aux autres taxons (Firmin, 1977) mais elle expliquerait la présence, dans ce niveau, du charme (Carpinus) qui n'apparaît qu'au Subatlantique dans le Bassin parisien (Leroyer, 1997).

Du fait de l'écrasante prédominance des Cichorioïdées, la zone 4 (éch. 7, 6 et 3) est difficilement interprétable. La disparition des céréales et la moindre diversité du cortège rudéral évoquent une baisse de la pression anthropique mais ces variations peuvent aussi résulter du biais taphonomique. Une interprétation environnementale reste, de toute façon, difficile puisque ces niveaux correspondent à des sédiments rapportés lors de la réalisation de la chape du tumulus qui peuvent contenir des pollens résiduels.

La zone 5 (éch. 5, 2 et 1) indique, à nouveau, la présence d'une petite chênaie à proximité du site tandis qu'une aulnaie réduite se maintient sur les berges du Crould. Ces boisements semblent, cependant, très clairsemés car les zones de prairies jouent un rôle important dans le paysage. Elles semblent pacagées au regard de la diversité du cortège rudéral mais aucune trace de céréaliculture n'est enregistrée. Les subdivisions internes illustrent quelques variations environnementales. Lors de la mise en place de l'horizon humifère (éch. 5), la chênaie et l'aulnaie apparaissent assez claires pour accueillir les ligneux héliophiles que sont les noisetiers et les lierres. La reprise des apports alluviaux (éch. 2) se marque par une très légère progression de l'aulnaie et surtout par un caractère beaucoup plus humide, voire marécageux des prairies. Celles-ci apparaissent fortement rudéralisées et semblent dévolues aux pâtures. Néanmoins, l'absence de tout enregistrement de céréaliculture ne permet pas d'exclure l'hypothèse que cette flore nitrophile relève d'un certain abandon des lieux. Ces zones de prairies humides restent présentes dans l'échantillon sommital mais elles ont évolué : la part de la caryçaie s'est réduite en faveur de formations amphibies plus diversifiées et des fougères. De plus, elles apparaissent moins rudéralisées. Toujours très clairsemés, les boisements attestent d'une modification majeure avec l'apparition du hêtre (Fagus) aux côtés des composants de la chênaie. Le début de cette zone apparaît subcontemporain du tumulus d'après la datation de l'horizon humifère $(3220$ +/- 110 BP) mais celui-ci peut correspondre au sol sous-jacent, rapporté à la surface de la structure pour «gazonnage » (Krier, supra). Si la présence du hêtre dans l'échantillon sommital incite à le considérer plutôt d'âge subatlantique, l'absence du charme et du châtaignier oriente vers un calage au début de la période.

\section{Discussions et CONCLUSIONS}

D'après les dates obtenues sur le site, la séquence pollinique de « La Fosse aux Larrons » couvre donc l'essentiel du Subboréal avec une première fréquentation des lieux durant le Néolithique final puis l'édification du tumulus durant le Bronze ancien. Il apparaît intéressant de la comparer au schéma général de l'histoire de la végétation régionale (Leroyer, 1997). Dans les séquences de fonds de vallées du Bassin parisien, le Subboréal (4700 à $2700 \mathrm{BP}$ ) est caractérisé par l'extension majeure de l'aulne, le retrait du tilleul au profit de l'orme et le maintien d'une chênaie diversifiée où le noisetier joue un rôle non négligeable. Le hêtre apparaît vers 4000 BP mais reste sporadique jusque vers 3000 BP. L'if (Taxus) est lui aussi présent très sporadiquement durant toute cette période. L'empreinte humaine se généralise alors avec une perception plus régulière des activités agro-pastorales des hommes de la fin du Néolithique et de l'âge du Bronze (Leroyer, 2003, 2006b ; Leroyer et Allenet, 2006). Des différences notables se marquent donc entre la séquence de Gonesse et ce référentiel. Outre l'abondance de Cichorioïdées, clairement liée au biais taphonomique, on constate également la sur-représentation du tilleul au détriment des autres ligneux (aulne, chêne, noisetier, orme...). La moindre représentation de l'aulne trouve sa justification dans la localisation des profils : son incidence étant majorée dans les paléochenaux aux bords desquels il pousse. Il en est tout autrement des composants de la chênaie qui devraient être mieux perçus à quelques distances des cours d'eau. Parmi ces derniers, seul le tilleul est prospère mais sa large prédominance à Gonesse s'oppose à la dynamique générale de la végétation qui le voit décroître dès le début du Subboréal après son optimum atteint durant l'Atlantique récent. Tilia est très souvent abondant dans les enregistrements de sites archéologiques depuis le Néolithique jusqu'à la fin de l'âge du Fer (Firmin, 1984). Ces constatations conduisent à se poser, là-encore, la question du biais taphonomique ; il est probable que le tilleul, type pollinique assez résistant à la corrosion, soit favorisé par l'altération du matériel. Enfin, les indices d'anthropisation restent effacés dans la séquence de Gonesse alors qu'ils sont mieux perçus à cette période même dans des profils éloignés de sites archéologiques (Leroyer, 2006 ; Leroyer et Allenet, 2006). Ainsi, la confrontation de la séquence pollinique de « La Fosse aux Larrons » avec le référentiel régional conforte la fiabilité réduite de ces données ; la forte corrosion du matériel et le remaniement anthropique lié à l'édification du tumulus induisent un biais taphonomique qui limite considérablement les interprétations. Seules quelques informations sur le paysage végétal et son anthropisation sont fournies par l'écologie des taxons présents mais leur incidence ne peut pas être prise en compte. En revanche, l'abondance du lierre dans l'échantillon prélevé sous le crâne du défunt pourrait éventuellement trouver sa justification dans une démarche cultuelle.

(C. Leroyer \& G. Firmin) 


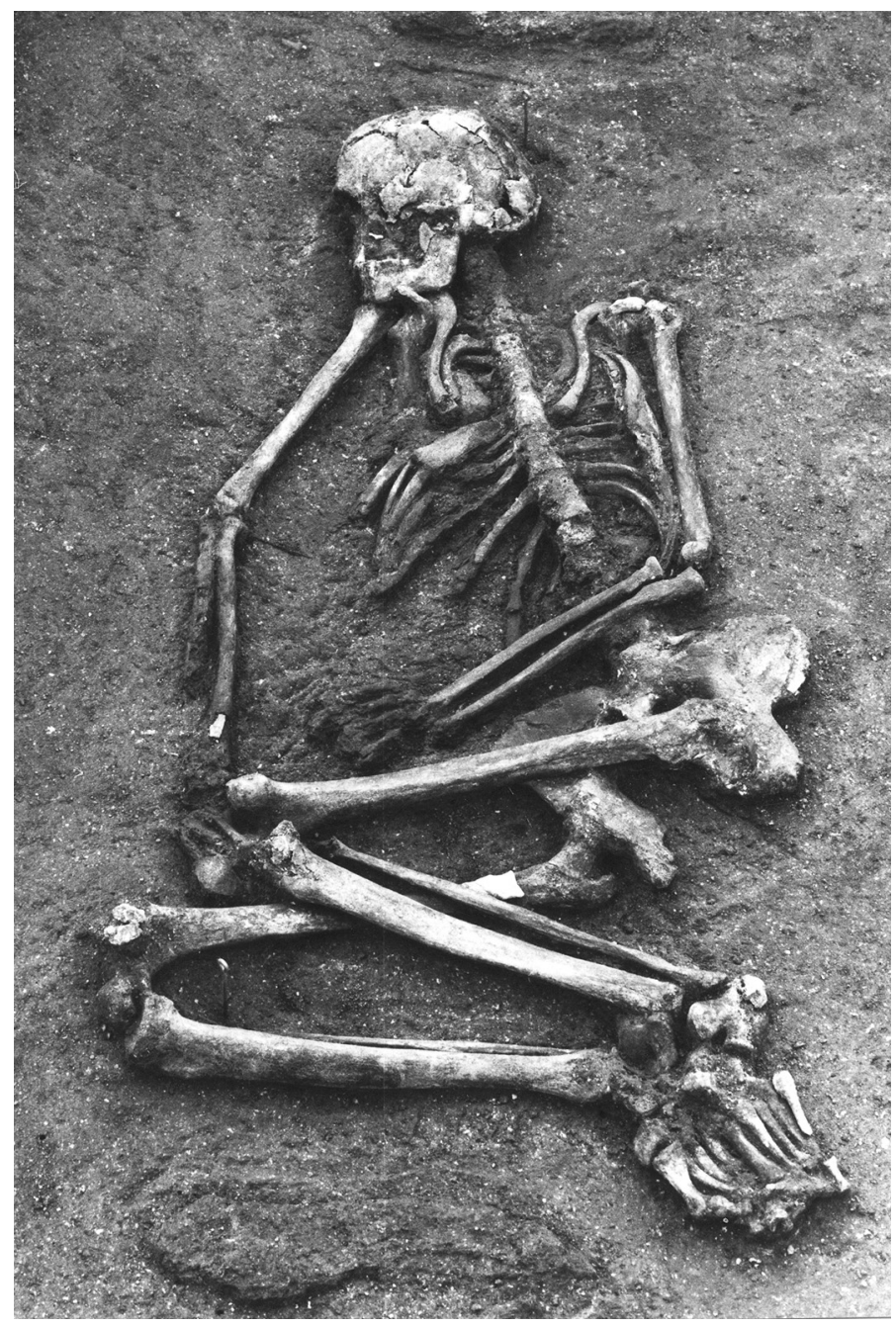

Fig. 11 - Gonesse (Val-d'Oise), La Fosse aux Larrons : vue subverticale de l'inhumation centrale; on distingue au-dessus de la tête et en dessous du tibia droit les éléments fusiformes ainsi que, derrière l'ulna gauche, la pointe en silex reposant sur le fémur droit à proximité de la tête fémorale.

Gonesse (Val-d'Oise), La Fosse aux Larrons: sub-vertical view of the central burial; spindle-shaped elements are visible above the head and below the right tibia, as well as the flint point behind the left ulna, resting on the right femur near the femoral head.

\section{LES TÉMOIGNAGES ARCHÉOLOGIQUES}

Les analyses environnementales dont les résultats affichent une bonne concordance permettent de caractériser les occupations pré- et protohistoriques qui se sont succédé dans cette partie de la plaine alluviale du Crould. La première est révélée par un ensemble de vestiges anthropiques (tessons de céramique, produits lithiques, ossements animaux) ou naturels modifiés (pierres brûlées) reconnu dans un niveau limoneux partiellement arasé surmontant un tuf de base. Ces artefacts témoignent de l'existence d'une zone d'habitat initiale qui a probablement subi un remaniement partiel par suite de reprises agricoles. Après le dépôt d'un nouvel horizon limoneux évoluant en un sol brunifié, c'est une fonction funéraire que connaît désormais cet espace anthropisé avec l'édification d'un grand tumulus.

Pour sa construction, un noyau de terre est constitué au détriment des sédiments environnants, prélevés parfois jusqu'au sommet du tuf et démantelant en partie le niveau contenant les vestiges de la première occupation humaine : c'est ce que leur examen détaillé (cf. infra) paraît bien montrer par comparaison avec ceux collectés dans le mince niveau limoneux surmontant le tuf. Pour contenir et stabiliser cette masse de terre, une couronne de pierres est ensuite ancrée dans le creusement périphérique résultant du prélèvement des sédiments.

Son dégagement ultérieur montra qu'elle était conservée sur une largeur variant de $1 \mathrm{~m}$ à $1,50 \mathrm{~m}$, formant une chape de 10 à $11 \mathrm{~m}$ de diamètre externe sans qu'on puisse savoir si elle recouvrait entièrement le tumulus à l'origine ou si elle le ceinturait seulement. Un échantillonnage de 100 pierres qui la constituait dans les mètres V / W 12 a été prélevé afin d'avoir un aperçu de leur nature et de leurs dimensions : 3 sont siliceuses, 2 en meulière, 4 en grès et les autres en calcaire. Les 3 plus grosses atteignent $40 \mathrm{~cm}$ de longueur, 3 se situent en-dessous de 10 $\mathrm{cm}, 34$ entre 16 et $20 \mathrm{~cm}, 45$ entre 10 et $15 \mathrm{~cm}$; quelques-unes atteignent et dépassent $10 \mathrm{~cm}$ d'épaisseur mais la plupart sont moitié moindres. Un apport de limons remaniés vient colmater cette ceinture de pierres : il est lui-même recouvert d'un mince niveau organique résultant peut-être de l'engazonnement de la surface du monument. Ainsi, avec ses chapes de terre et de pierres, l'ensemble du tumulus, à peu près circulaire, devait avoir un diamètre total d'environ $16 \mathrm{~m}$.

\section{LA SÉPULTURE}

\section{DisPosition des ReStes OSSEUX ET MODE D'ENSEVELISSEMENT}

Le corps reposait à plat si ce n'est un enfoncement de quelques centimètres du bassin et du crâne par rapport au reste du squelette ; celui-ci était sur le dos, la face tournée vers la droite, le membre supérieur droit allongé, le gauche ramené sur l'abdomen, les membres inférieurs repliés sur le côté droit, le fémur gauche ramené perpendiculairement à la colonne vertébrale (fig. 11). A une dizaine de centimètres en-dessous des tibia et ulna droits et parallèlement à eux dans une zone de terre plus foncée s'allongeait sur une trentaine de centimètres quelque chose de fusiforme à la texture fibreuse qu'on retrouvait sous une forme analogue à quelque distance du crâne : aucune détermination n'a pu en être faite en laboratoire. En arrière de l'ulna gauche au-dessus de la tête du fémur droit se trouvait une pointe en silex sommairement aménagée qui pourrait avoir accompagné le défunt, aucun autre vestige n'ayant été recueilli à proximité (fig. 19, n 11). Une observation plus détaillée des ossements montre une mise à plat générale du squelette, aucune pièce ne se trouvant en déséquilibre sauf peut-être l'humérus gauche. On constate plusieurs dislocations qui ont conduit des ossements à sortir du volume initial du cadavre. On relève ainsi un affaissement des côtes avec du côté droit une ouverture excédant le volume initial du thorax ; au niveau des membres supérieurs, il y a rupture du poignet gauche marquée par un décalage entre l'avant-bras et les métacarpiens qui traduit un glissement vers le bas des os de l'avant-bras ; au niveau du bassin, on note un affaissement et un recul de l'os coxal gauche ainsi qu'un léger affaissement de l'os coxal droit ; quant aux fémurs, ils ont effectué une rotation au niveau des genoux, le fémur gauche se trouvant en face postéro-latérale, le droit en face postérieure ; enfin, la fibula gauche est affaissée en arrière du tibia avec un écartement au niveau proximal. Ces 

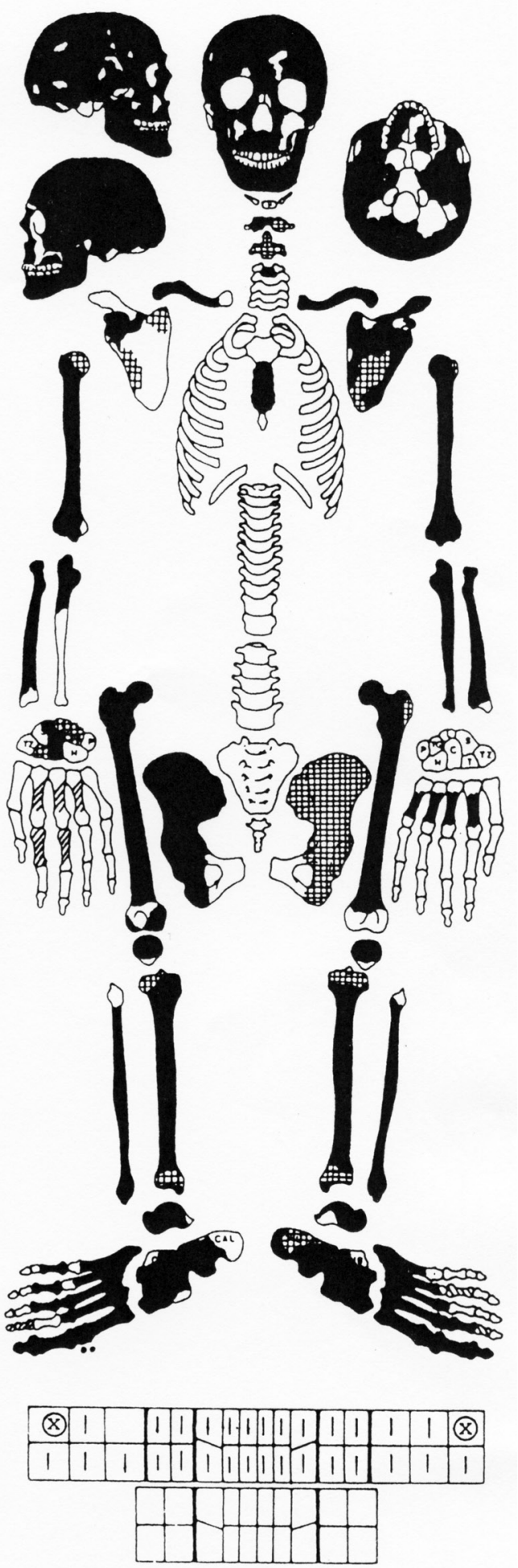

diverses dislocations indiquent à chaque emplacement un vide au moment de la décomposition ce qui invite à l'explication globale d'une décomposition de l'ensemble du corps en espace non colmaté. Dans la mesure où la disposition des ossements ne révèle pas de contraintes sur le corps, ni compression ni effet de paroi, ce contenant devait être suffisamment spacieux. Peut-on avoir une idée de sa forme ? Les seuls indices qui subsistent sont ces deux éléments fusiformes disposés parallèlement au-dessus de la tête et sous les pieds, en association avec un sédiment plus sombre observé sous l'élément inférieur et présentant une limite
Fig. 12 - Gonesse (Val-d'Oise), La Fosse aux Larrons : conservation du squelette cotée selon la méthode de Meiklejohn et ConstandseeWestermann : en noir, la région est présente et identifiée avec certitude ; en quadrillé, l'os est présent mais fragmenté et/ou érodé et lou écrasé ; en hachuré, fragments très incertains quant au côté et/ ou à la nature, reconnus simplement par leur emplacement à la fouille. Légende du diagramme dentaire : barre verticale : présence in situ ; croix cerclée : agénésie dentaire.

Gonesse (Val-d'Oise), La Fosse aux Larrons: preservation of the skeleton listed according to the method by Meiklejohn and ConstandseeWestermann: in black, the region is present and identified with certainty; in cross-hatching, the bone is present but fragmented and/or eroded and/ or crushed; in hatching, very uncertain fragments as regards side and/or nature, identified simply by their position during excavation. Caption for dentition: vertical bar: presence in situ; encircled cross: dental agenesis.

rectiligne quelques centimètres plus bas : sans doute peut-on y voir la possibilité d'un vraisemblable contenant rectangulaire assez large.

Rappelons que l'étude anthropologique qui suit se présente de manière extrêmement détaillée telle qu'il était d'usage de la réaliser à l'époque de sa rédaction.

(J. Tarrête)

\section{ÉtUdE ANTHROPOLOGIQUE}

Létat de conservation des vestiges est relativement satisfaisant (fig. 12) ; on note cependant de nombreuses érosions mécaniques, des fragmentations multiples avec pertes de substance, ainsi que des vermiculations superficielles dues à l'action lytique des racines. Il existe en outre de très nettes traces de dents de rongeur sur la diaphyse du cinquième métatarsien gauche.

Le squelette des extrémités est assez mal représenté, notamment en ce qui concerne les mains. Par ailleurs, nous n'avons pu examiner les côtes et la plupart des vertèbres, qui ont servi à la datation isotopique de la tombe.

\section{Détermination du sexe}

Nous avons utilisé dans ce but les méthodes proposées par D. Ferembach, I. Schwidetsky et M. Stloukal (Ferembach et al., 1979).

Le coxal droit, dont le pubis est détruit, a un coefficient de sexualisation de $+0,86(+12 / 14)$, ce qui indique une morphologie androïde moyennement accusée (tabl. III). L'indice cotylo-sciatique, qui rapporte la hauteur de l'incisura ischiadica major $(40,5 \mathrm{~mm})$ à la largeur cotylo-sciatique $(38 \mathrm{~mm})$ est faible $(106,6)$, et confirme donc la masculinité de ce sujet.

Nous avons également relevé les caractères « sexuels » du squelette céphalique (tabl. IV). Le coefficient de sexualisation est plus élevé $(+30 / 23=+1,30)$.

Il paraît donc bien établi que cette sépulture est celle d'un individu de sexe masculin.

\section{Détermination de l'âge au décès}

Il s'agit indiscutablement d'un squelette d'adulte : les épiphyses des os longs sont toutes entièrement soudées aux diaphyses, y compris l'extrémité interne de la clavicule. Par ailleurs, les troisièmes molaires sont en place sur la mandibule. 


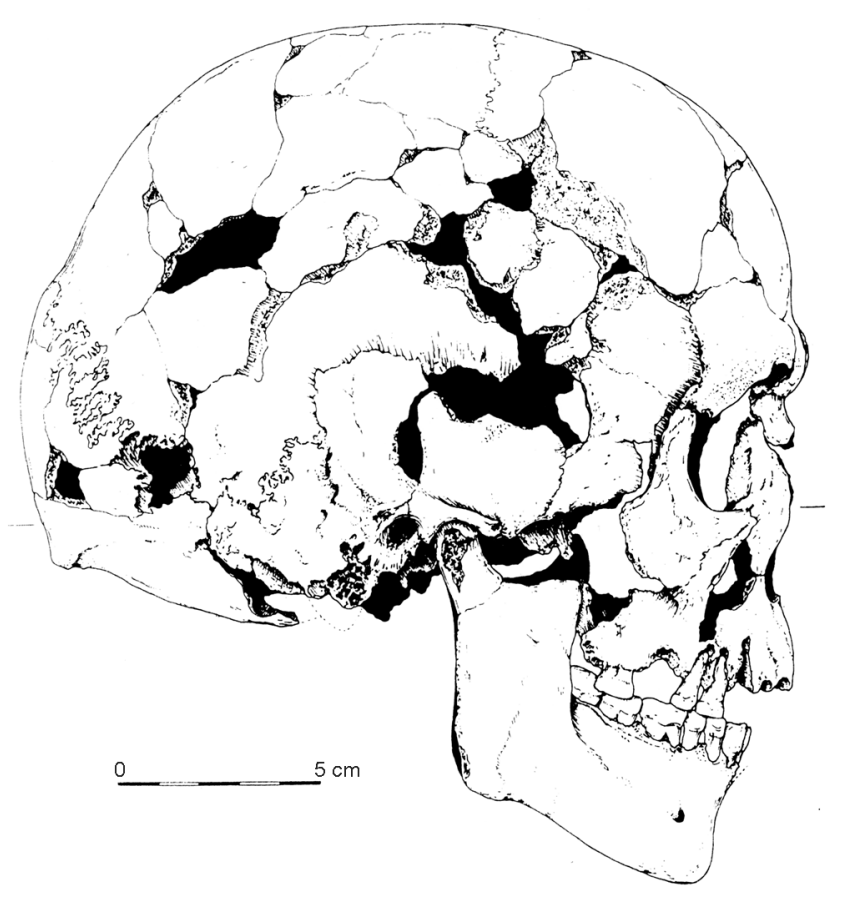

Fig. 13 - Gonesse (Val-d'Oise), La Fosse aux Larrons : vue latérale droite du squelette céphalique.

Gonesse (Val-d'Oise), La Fosse aux Larrons: right lateral view of the head bones.

Les sutures crâniennes sont presque entièrement synostosées sur la face endocrânienne, à l'exception de la lambdoïde droite dans sa portion médiane (pars lambdica) et de la coronale gauche, également dans sa portion médiane (pars bregmatica). Sur la face exocrânienne, l'oblitération est évidemment moins avancée. Elle est néanmoins très nette sur toute la coronale (stade 2 de Broca), sur la sagittale (stade 1), à l'exception de la pars verticis qui est libre, et sur toute la lambdoïde gauche (stade 1).

Depuis les recherches de C. Masset (Masset, 1982), on sait qu'il ne faut pas accorder une confiance absolue à ces critères et surtout que l'on doit se garder de vouloir assigner à un squelette isolé un âge trop précis. Néanmoins, un tel schéma suggère un adulte d'âge moyen ou avancé, en tout cas supérieur à trente ans.

L'usure dentaire est moyenne (stade 2 de Brabant et Sahly) pour toutes les dents, à l'exception des troisièmes molaires inférieures (stade 1); cette constatation ne peut toutefois être tenue pour le signe d'un décès précoce, dans la mesure où ce sujet présente une agénésie des troisièmes molaires supérieures.

\section{Étude ostéométrique et ostéoscopique.}

\section{Le squelette axial.}

Squelette céphalique (Ossa capitis).

\section{Bloc crânio-facial}

Le crâne a pu être reconstitué de manière satisfaisante, à quelques défauts qui affectent essentiellement le splanchnocrâne. Les mensurations en sont indiquées au tabl. V.
Il s'en dégage une impression générale de robustesse. Le neurocrâne est volumineux (aristencéphale) ; les sutures, là où elles sont encore lisibles, ont un dessin plutôt simple, à l'exception de la lambdoïde dont les indentations très serrées s'étalent sur une largeur de $20 \mathrm{~mm}$ environ, emprisonnant de très nombreux os wormiens étroits et allongés. Il existait un os interpariétal mesurant approximativement $38 \mathrm{~mm}$ de large sur $19 \mathrm{~mm}$ de haut.

En norma verticalis, le crâne est ovoïde, long. La glabelle, quoique saillante, est déprimée entre deux puissants massifs sourciliers. Le front est moyennement divergent, et les arcades zygomatiques, brisées, sont à peine visibles. Les bosses frontales sont bien marquées, les bosses pariétales plus effacées.

En norma lateralis, la voûte est haute, fortement bombée. Après un méplat lambdatique, l'écaille occipitale est projetée en bas et en arrière, formant un véritable « chignon ». La région glabellaire, bien qu'endommagée, est en forte saillie (type IV de Martin). Le front est plutôt droit. La protubérance occipitale externe est extrêmement puissante, dessinant un véritable bec dirigé vers le bas (type V de Broca). Les processus mastoïdes, quoique brisés, paraissent larges et moulurés. L'épine supra meatum est nette, la crête sus-mastoïdienne renflée en un tubercule que bordent en arrière plusieurs dépressions irrégulières aboutissant à l'incisure pariétale.

Les régions ptériques sont brisées ; les lignes temporales ont un relief moyen (mais l'os est érodé à leur niveau). Les tubercules zygomatiques postérieurs sont hauts et forment le mur postérieur de la cavité glénoïde.

La face est haute et verticale (orthognathe). L'ensellure nasale est peu accentuée, l'épine nasale antérieure est brisée (fig. 13).

En norma facialis, on retrouve la puissance des reliefs susorbitaires. La face est haute, très étroite. Les orbites, hautes et arrondies, sont limitées par un rebord émoussé. Les os zygomatiques sont également hauts, assez moulurés. Le nez paraît être de hauteur et de largeur moyennes, mais la reconstitution est défectueuse dans cette région.

En norma occipitalis, on note une forte carène sagittale. La largeur transverse maximale se situe au niveau des bosses pariétales. La protubérance occipitale externe paraît énorme, développée en une languette large, irrégulière, piquetée de pertuis vasculaire, qui vient recouvrir le tuberculum linearum.

La base du crâne est très endommagée, de sorte que la norma basilaris ne permet aucune observation pertinente.

En conclusion, ce sujet est aristencéphale, dolichocrâne, hypsicrâne, acrocrâne, a un indice mixte de hauteur élevé, des crêtes frontales intermédiaires à tendance divergente, métriométope, hyperleptoprosope, hyperleptène, hypsiconque, mésorhinien (?) et orthognathe.

\section{Mandibule (Mandibula)}

La mandibule est également puissante, massive. L'angle goniaque est moyen, les branches montantes assez larges mais hautes, de sorte que leur indice n'est pas très élevé. Le processus coronoïde, du type styliforme de Le Double, est plus haut que le condyle. Les reliefs d'insertion musculaires sont visibles, mais émoussés. On note cependant une légère extroversion des régions goniaques. L'incisure préangulaire est accusée, il n'y a pas d'incisure sous-mentale. La lingula est large, peu saillante, et il n'y a pas d'antilingula. Le sillon 
mylo-hyoïdien est large, assez mal délimité. La ligne mylohyoïdienne n'est bien définie que dans sa portion postérieure, sous les deuxièmes et troisièmes molaires.

Les trous mentonniers sont grands, situés sous l'intervalle P1-P2. Les apophyses géni sont du type IV1b de Heim $($ Heim,1963) et sont surmontées par un trou borgne sur la ligne médiane.

Les dimensions de la mandibule sont données au tabl. VI.

\section{Dents (Dentes)}

Les mesures des dents sont indiquées dans le tabl. VII. Diverses anomalies ont pu être relevées sur les dents et le parodonte : elles sont exposées au chapitre de la paléopathologie.

Pour chaque dent, on a successivement indiqué le diamètre mésio-distal et le diamètre vestibulo-lingual.

\section{Squelette du tronc}

A l'exception de quelques fragments se rapportant aux premières cervicales, le squelette du tronc se limite au corps sternal.

\section{Sternum}

Les dimensions du corps sternal sont données au tabl. VIII. Il est très asymétrique, les échancrures costales gauches étant beaucoup plus profondes que les droites et nettement décalées vers le bas : le bord supérieur de la troisième échancrure costale droite se trouve $5 \mathrm{~mm}$ plus haut que le gauche. En outre, le bord latéral gauche du corps est légèrement concave, le droit convexe.

\section{Le squelette appendiculaire.}

\section{Membres supérieurs.}

\section{Clavicule (Clavicula)}

Les clavicules sont moyennement flexueuses. Le tubercule conoïde est peu saillant, la tubérosité coracoïdienne remplacée par une empreinte irrégulière bordée vers l'arrière par une crête en léger relief. Il n'y a pas à proprement parler de tubérosité costale : aucun relief n'est visible à son niveau du côté gauche, alors qu'il existe à droite quelques rugosités. L'insertion du muscle sous-clavier est marquée par un méplat.

Les dimensions des clavicules sont regroupées dans le tabl. IX.

\section{Scapula (Scapula)}

Seule est étudiable la scapula gauche, la droite étant trop incomplète. Elle est robuste, avec un pilier puissant, mouluré. Sur l'épine, le tubercule d'insertion du muscle trapèze est bien dégagé. Des deux côtés, la cavité glénoïde est du type piriforme.

Les dimensions des scapulas sont données au tabl. X.

\section{Humérus (Humerus)}

Les humérus sont longs, robustes, et ont une épiphyse distale large et forte. La section au milieu de l'os est sensiblement rectangulaire. Le gauche est plus aplati : l'indice diaphysaire indique la platybrachie, alors que le droit se situe à la limite de l'eurybrachie (tabl. XI).

\section{Radius (Radius)}

Les diaphyses radiales sont érodées, de sorte que la ligne interosseuse a un aspect quelque peu émoussé. A gauche, l'os est très faiblement incurvé, avec un col épais et peu incliné (tabl. XII).

\section{UInas $(U \ln a)$}

Ici encore, seule l'ulna gauche est étudiable. L'os est robuste, mouluré. On note une petite crête discontinue, d'aspect non articulaire, à la jonction des versants horizontal et vertical de l'incisure trochléaire. Le même aspect peut être observé à droite, malgré l'érosion de l'os (tabl. XIII).

\section{Squelette des mains}

Les quelques vestiges conservés (carpiens, métacarpiens et phalanges) sont trop endommagés pour autoriser la moindre observation et, a fortiori, la moindre mesure.

\section{Membres inférieurs.}

\section{Os coxal (Os coxae)}

Le coxal gauche est complètement écrasé et n'a pu être reconstitué. Le droit est mieux conservé. Nous avons fait mention de sa morphologie et de quelques-unes de ses dimensions au chapitre de la diagnose sexuelle et ne reviendrons pas sur ces points.

La largeur de l'ilion est de $162 \mathrm{~mm}$, et la hauteur de l'os coxal devait être très nettement supérieure à $220 \mathrm{~mm}$ (environ $240 \mathrm{~mm})$

\section{Fémur (Os femoris)}

Les fémurs sont dans l'ensemble assez bien conservés, excepté au niveau de leur extrémité distale. La diaphyse est nettement plus large du côté gauche. La ligne âpre est forte, rugueuse mais mal dégagée (tabl. XIV).

On note un très important aplatissement antéro-postérieur de la région hypotrochantérienne (hyperplatymérie). Il existe en outre, surtout du côté droit, une ébauche de gouttière hypotrochantérienne. Au niveau de la tête, il n'y a pas de zone d'Allen ; par contre, de chaque côté, la zone de Poirier est très accusée.

\section{Patella (Patella)}

Les rotules semblent hautes, parce que leur face supérieure est oblique en haut et en arrière. Elles paraissent peu moulurées, mais leur surface est érodée. L'angle supéro-externe n'est visible que du côté gauche : il est à peine ourlé, sans véritable vastus notch (Tabl. XV).

\section{Tibia (Tibia)}

Les quelques dimensions qui ont pu être prises sont présentées dans le tabl. XVI. La section diaphysaire est du type III de Hrdlicka, mais la face antéro-externe est peu excavée. La crête tibiale est peu flexueuse, et les reliefs d'insertion musculaire sont peu accentués, notamment la ligne oblique. Néanmoins, ces os ont un aspect général massif.

On note une facette surnuméraire latérale du côté gauche (facette dite « d'accroupissement » ou facette « orientale »). L'extrémité distale du tibia droit n'est pas conservée dans sa portion antérieure, ce qui interdit la recherche de ce caractère. 


\section{Fibula (Fibula)}

Les rares mensurations qu'il a été possible d'effectuer sont données au tabl. XVII. On retiendra seulement que les diaphyses sont fortement cannelées.

\section{Talus (Talus)}

Seul est convenablement conservé le talus gauche (tabl. XVIII). Les surfaces articulaires surnuméraires du col sont à peine ébauchées.

\section{Calcanéus (Calcaneus)}

Les calcanéus sont très incomplets. La surface articulaire antéro-supérieure pour le talus est unique, mais bilobée. Les seules mensurations possibles concernent la surface articulaire postéro-supérieure pour le talus, dont la longueur est de $32 \mathrm{~mm}$ à gauche, $31 \mathrm{~mm}$ à droite, et la largeur de $25 \mathrm{~mm}$ de chaque côté.

\section{Os naviculaire (Os naviculare) (tabl. XIX)}

Os cunéiformes (Os cuneiforme mediale, intermedium et laterale).

Les cunéiformes sont bien conservés. Leurs dimensions sont indiquées dans les tabl. XX et XXI.

\section{Métatarsiens (Metatarsus)}

Les métatarses sont également presque complets (tabl. XXII). Seul n'a pu être mesuré le cinquième métatarsien gauche, dont il ne subsiste qu'un fragment de la diaphyse, attaqué par les rongeurs.

\section{Phalanges du pied (Phalenges pedis)}

Nous n'avons mesuré que les phalanges de rayon et de côté connus, c'est-à-dire les phalanges des hallux (tabl. XXIII).

\section{Détermination de la stature}

Dans le tabl. XXIV, nous avons regroupé les résultats obtenus en utilisant les tables classiques de détermination de la stature à partir de la longueur des grands os longs des membres. Les valeurs oscillent entre 165 et $175 \mathrm{~cm}$, et nous proposons une estimation réalisée à partir des longueurs combinées du fémur et du tibia, soit $170 \mathrm{~cm}$. Il s'agit donc d'un homme de taille relativement élevée, surtout si l'on se réfère aux populations de la Préhistoire récente et du début des Ages des Métaux qui ont été étudiées en France (Riquet, 1970).

\section{Paléopathologie}

Les seules lésions que nous ayons relevées affectent des dents et le parodonte. Elles représentent un échantillonnage assez varié des divers chapitres de la pathologie alvéolo-dentaire.

Agénésie : on note une agénésie des troisièmes molaires supérieures ; les troisièmes molaires inférieures sont par contre en place.

Dysplasies de l'émail : il s'agit en fait d'une hypoplasie linéaire de l'émail, qui se traduit par la présence de sillons horizontaux, parallèles au bord libre de la dent. Il peut n'exister qu'un sillon, mais certaines dents montrent plusieurs sillons superposés (jusqu'à quatre au niveau des canines). Ces lésions sont plus ou moins complètement masquées par un dépôt de tartre (cf. infra) ; elles affectent essentiellement la face vestibulaire (tabl. XXV), mais sur les canines et les premières prémolaires, elles se prolongent sur les faces latérales (et même sur la face linguale des canines).

L'incisive médiane inférieure droite présente en outre sur sa face vestibulaire une fossette allongée selon l'axe longitudinal de la dent, qui s'étend du tiers inférieur de la couronne au bord occlusal et confère à la dent un aspect bosselé tout à fait inhabituel.

Malgré leur netteté, ces lésions sont cependant limitées : en aucun point, la dentine n'est mise à nu. Elles doivent être considérées comme une forme mineure de dysplasie. De telles malformations résultent d'un défaut de l'adamantogenèse qui peut survenir à l'occasion de n'importe quelle agression affectant l'organisme d'un jeune enfant. Les étiologies les plus courantes (Dechaume, 1966) en sont actuellement les maladies aigües (infectieuses surtout) ou chroniques avec poussées aigües (troubles gastro-intestinaux, avitaminoses, toxi-infections, rachitisme, syphilis congénitale...). Il n'est évidemment pas possible, en l'occurrence, de trancher entre ces diverses causes...

Malpositions dentaires : il existe des signes patents d'encombrement au niveau de l'arcade inférieure, avec chevauchement de l'incisive latérale gauche, qui est légèrement déjetée dans le sens lingual, de la canine gauche, plus fortement basculée en direction vestibulaire, et de la première prémolaire gauche, elle aussi décalée en direction linguale. Les dents sont mieux rangées à droite, où les facettes d'usure interdentaire sont beaucoup plus accentuées qu'à gauche.

Caries : nous avons observé de multiples caries du collet, sur la face distale de la première prémolaire inférieure droite, la face mésiale de la deuxième prémolaire inférieure droite, la face distale de la première molaire inférieure gauche, la face mésiale de la deuxième molaire inférieure gauche, la face mésiale de la première prémolaire supérieure droite et la face mésiale de la première molaire supérieure gauche (cette dernière étant très limitée).

Les lésions les plus sévères affectent la première prémolaire supérieure droite et la première molaire inférieure gauche : la cavitation a entrainé l'effondrement d'une partie de la face occlusale (à l'aplomb de la carie), et s'accompagne d'une excavation de l'os alvéolaire au niveau de l'apex, avec béance sur la face vestibulaire. Cette anomalie est centrée sur la racine distale de la molaire, de sorte qu'il n'a pas été possible de mesurer le périmètre du corps mandibulaire entre les deux premières molaires inférieures gauches. Comme il n'existe pas à ce niveau de signes évidents d'hypervascularisation, l'hypothèse la plus plausible est qu'il s'agit de granulomes (ou kystes) apicaux, et non de véritables lésions d'ostéite septique.

Parodontopathies : outre les deux foyers apicaux que nous venons d'évoquer, on note un dépôt massif de tartre sur toutes les dents. Il prédomine sur la face vestibulaire, à laquelle il est limité en ce qui concerne les dents supérieures.

D'autre part, partout où il est conservé, l'os alvéolaire paraît festonné, piqueté de pertuis vasculaires. La résorption est manifeste, comme en témoigne le déchaussement étendu des racines. De telles lésions signent un processus de gingivite chronique, probablement à mettre en rapport avec le dépôt diffus de tartre. 
On notera enfin que l'alvéole de la première molaire supérieure droite est béant, excavé, et montre de nombreux trous vasculaires. Bien que l'os ait été endommagé post mortem, l'anomalie est évidente et son caractère pathologique indiscutable. Il pourrait s'agir d'une ostéite ayant abouti à l'expulsion de la dent, car celle-ci est la seule qui n'ait pas été retrouvée à la fouille.

\section{Conclusions}

Le tumulus de « La Fosse aux Larrons » à Gonesse (Vald'Oise), daté du début du Bronze moyen (circa 1500 av. J.-C.), a livré le squelette presque complet d'un individu adulte, de sexe masculin, décédé à un âge certainement supérieur à trente ans. Les lésions pathologiques que nous avons relevées concernent exclusivement les dents et le parodonte, mais elles sont remarquablement diverses et étendues.
Sa taille relativement élevée (environ $170 \mathrm{~cm}$ ) et ses caractères morphologiques peuvent suggérer certains rapprochements comme, par exemple, avec le sujet découvert dans le tumulus de «La Charme au Moulin » à Villecomte (Côte-d'Or) d'âge plus tardif (Chabeuf, 1967). Il n'est évidemment pas question d'entreprendre, à l'occasion de l'étude d'un individu isolé, une révision des données anthropologiques concernant le Bronze moyen - récent. De fait, une telle entreprise serait de toute manière prématurée, tant manquent à ce jour les éléments de référence convenablement datés, conservés et étudiés...

Tabl. III - Détermination du sexe à partir de l'os coxal droit.

Sex determination from the right coxal bone.

\begin{tabular}{|c|c|c|c|c|c|c|c|}
\hline Caractère & Poids & Hyper féminin - 2 & Féminin - 1 & Inter-médiaire 0 & Masculin + 1 & Hyper masculin +2 & $\mathrm{x}$ \\
\hline Sulcus praeauricularis & 3 & & & & & + & +6 \\
\hline Angulus pubis & (2) & & & & & & \\
\hline Os coxae & 2 & & & & + & & +2 \\
\hline Foramen obturatum & (2) & & & & & & \\
\hline Corpus ossis ischii & 2 & & & + & & & 0 \\
\hline Crista iliaca & 1 & & & & + & & +1 \\
\hline Pelvis minor & (1) & & & & & & \\
\hline TOTAL & 14 & & & & & & +12 \\
\hline
\end{tabular}


Tabl. IV - Détermination du sexe à partir du squelette céphalique. Sex determination from the cephalic skeleton.

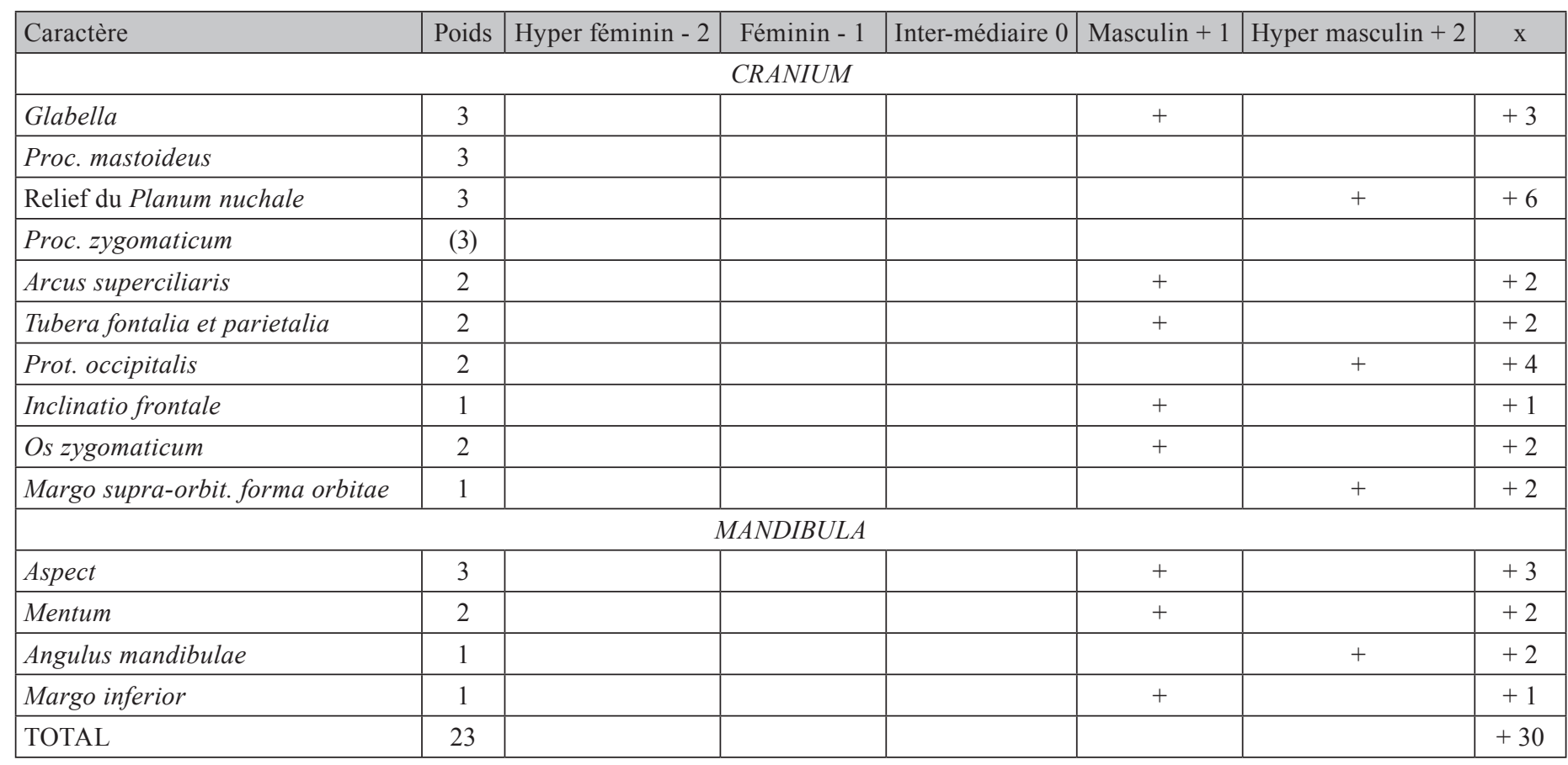

Tabl. Vbis - Indices du crâne.

\section{Skull indexes.}

\begin{tabular}{|l|l|}
\hline Indice crânien horizontal (I1, 8/1) & $\# 74.5$ \\
Indice de Hauteur-Longueur, Basion (I2, 17/1) & - \\
Indice de Hauteur-Longueur, Porion (I4, 20/1) & 64.3 \\
Indice de Hauteur-Largeur, Basion (I3,17/8) & - \\
Indice de Hauteur-Largeur, Porion (I5, 20/8) & $\# 86.3$ \\
Indice mixte de Hauteur, Basion (I7/1+8) & - \\
Indice mixte de Hauteur, Porion (20/1+8) & $\# 73.7$ \\
Indice de Hauteur de la calotte (I5(1), 22a/2) & 59.8 \\
Indice du foramen magnum (I33, 16/7) & - \\
Indice frontal transversal (I12, 9/10) & 80.3 \\
Indice fronto-pariétal transversal (I13, 9/8) & $\# 67.1$ \\
& \\
Indice crânio-facial transversal (I71, 45/8) & $\# 85.6$ \\
Indice fronto-goniaque (66/9) & 98.0 \\
Indice facial total (I38, 47/45) & $\# 100.8$ \\
Indice facial supérieur (I39, 48/45) & 60.0 \\
Indice orbitaire D - G (I42, 52/51) & $89.5-\mathrm{x}$ \\
Indice nasal (I48, 54/55) & $\# 49.0$ \\
Indice palatin (I58, 63/62) & - \\
Indice maxillo-alvéolaire (I54, 61/60) & - \\
Indic gnathique de Flower (I60, 40/5) & 90.9 \\
Indice frontal sagittal (I22, 29/26) & 75.5 \\
Indice pariétal sagittal (I24, 30/27) & - \\
Indice occipital sagittal (I25, 31/28) & \\
\hline
\end{tabular}


Tabl. V - Caractères métriques du crâne.

Skull metrical characters.

\begin{tabular}{|c|c|}
\hline Longueur maximale (1) & 196 \\
\hline Longueur Glabelle-Inion (2) & 189 \\
\hline Largeur transverse maximale (8) & \# 146 \\
\hline Largeur frontale minimale (9) & 98 \\
\hline Largeur frontale maximale (10) & 122 \\
\hline Diamètre stéphanique (10b) & 121 \\
\hline Largeur biastérique (12) & 109 \\
\hline Largeur biauriculaire (11) & 116 \\
\hline Hauteur Basio-Bregmatique (17) & - \\
\hline Hauteur Porio-Bregmatique (20) & 126 \\
\hline Périmètre horizontal (23) & 551 \\
\hline Périmètre frontal (24) & 339 \\
\hline Périmètre sagittal (25) & 405 \\
\hline Arc frontal (26) & 133 \\
\hline Corde frontale (29) & 116.5 \\
\hline Arc pariétal (27) & 132 \\
\hline Corde pariétale (30) & 120 \\
\hline Arc occipital (28) & 139 \\
\hline Corde occipitale (31) & 105 \\
\hline Longueur du foramen magnum (7) & - \\
\hline Largeur du foramen magnum (16) & - \\
\hline Hauteur de la mastoïde D - G & $X-X$ \\
\hline Hauteur totale de la face (47) & \# 126 \\
\hline Hauteur faciale supérieure (48) & 75 \\
\hline Largeur bizygomatique (45) & \# 125 \\
\hline Largeur bijugale (45(1)) & 109 \\
\hline Largeur bimaxillaire maximale (46) & - \\
\hline Longueur de la face (40) & - \\
\hline Longueur de la base (5) & - \\
\hline Hauteur du nez (55) & 51 \\
\hline Largeur du nez (54) & $\# 25$ \\
\hline Largeur de l'orbite D - G (51) & $38-X$ \\
\hline Hauteur de l'orbite D - G (52) & $34-X$ \\
\hline Largeur biobitaire (44) & \# 89 \\
\hline Largeur interorbitaire antérieure (50) & 18 \\
\hline Longueur du palais (62) & \# 48 \\
\hline Largeur du palais (63) & - \\
\hline Longueur maxillo-alvéolaire (60) & \# 52 \\
\hline Largeur maxillo-alvéolaire (61) & \# 58 (2 X D) \\
\hline Hauteur de la calotte (22a) & 113 \\
\hline \multicolumn{2}{|l|}{ Angle de profil total (72) } \\
\hline Angle de profil nasal (73) & $90^{\circ}$ \\
\hline Angle de profil alvéolaire (74) & $91^{\circ}$ \\
\hline \multirow[t]{2}{*}{ Angle facial de Weisbach (72(5)) } & $85^{\circ}$ \\
\hline & - \\
\hline Angle frontal de Schwalbe (32(2)) & $65^{\circ}$ \\
\hline Capacité crânienne (Manouvrier) (38c) & - \\
\hline Capacité crânienne (Lee et Pearson) (38d, Basion) & - \\
\hline Capacité crânienne (Lee et Pearson) (38d, Porion) & 1675 \\
\hline Capacité crânienne (Welcker) (38a) & - \\
\hline
\end{tabular}

Tabl. VI - Caractères métriques de la mandibule.

Mandible metric characters.

\begin{tabular}{|c|c|}
\hline Longueur totale (68(1)) & 101 \\
\hline Largeur bicondylienne (65) & $>119$ (\# 125) \\
\hline Largeur bigoniaque (66) & \# 96 \\
\hline Largeur bimentale (67) & 44 \\
\hline Largeur bocoronoïdienne (65(1)) & \# 102 \\
\hline Largeur de l'arcade & 60.5 \\
\hline Longueur de l'arcade & 51 \\
\hline Périmètre de l'arcade & 139 \\
\hline Angle goniaque (79) & $129^{\circ}$ \\
\hline Angle symphysien (79(1a)) & $\mathrm{x}$ \\
\hline Angle symphysien (Pogonion-Infradental/base) & $\mathrm{x}$ \\
\hline Angle intercondylien (79(5)) & $\mathrm{x}$ \\
\hline Hauteur de la branche D - G (70) & $66.5-x$ \\
\hline Largeur de la branche D - G (71) & \# $31-33$ \\
\hline Hauteur du corps & \\
\hline symphyse & \# 35 \\
\hline P1-P2 & $36-35$ \\
\hline P2-M1 & $34.5-34$ \\
\hline M1-M2 & $33-x$ \\
\hline M2-M3 & $30.5-30$ \\
\hline Epaisseur réelle du corps & \\
\hline symphyse & 14 \\
\hline P1-P2 & $11.5-12$ \\
\hline P2-M1 & $11.5-11.5$ \\
\hline M1-M2 & $12-12.5$ \\
\hline M2-M3 & $14-14$ \\
\hline Périmètre du corps & \\
\hline symphyse & $\mathrm{x}$ \\
\hline P1-P2 & $83-82$ \\
\hline P2-M1 & $82-81$ \\
\hline M1-M2 & $79-x$ \\
\hline M2-M3 & $79-78$ \\
\hline Longueur du condyle $\mathrm{D}-\mathrm{G}$ & $x-x$ \\
\hline Largeur du condyle $\mathrm{D}-\mathrm{G}$ & $10-x$ \\
\hline Longueur du corps & 74 \\
\hline Indice mandibulaire $(\mathrm{I} 62,68(1) / 65)$ & \# 80.8 \\
\hline Indice gonio-condylien (I64, 66/65) & \# 76.8 \\
\hline Indice de la branche montante $\mathrm{D}-\mathrm{G}(\mathrm{I} 63,71 / 70)$ & $51.2-\mathrm{x}$ \\
\hline
\end{tabular}


Tabl. VII - Caractères métriques des dents.

Teeth metric characters.

\begin{tabular}{|l|c|c|c|c|c|}
\hline \multicolumn{3}{|c|}{ Dents supérieures } & \multicolumn{3}{c|}{ Dents inférieures } \\
\hline & & D & & D & G \\
\hline I1 & $9.0-7.5$ & $8.8-7.2$ & I1 & $5.5-5.6$ & $5.5-5.7$ \\
\hline I2 & $6.9-6.1$ & $7.1-6.5$ & I2 & $6.2-6.2$ & $6.5-6.6$ \\
\hline C & $7.7-8.5$ & $7.8-8.4$ & C & $7.4-7.6$ & $7.3-8.0$ \\
\hline P1 & $7.1-9.5$ & $7.0-9.8$ & P1 & $6.7-7.8$ & $7.4-7.7$ \\
\hline P2 & $\mathrm{x}-9.3$ & $6.5-9.2$ & P2 & $6.7-8.7$ & $6.6-8.4$ \\
\hline M1 & $\mathrm{x}-\mathrm{x}$ & $11.0-12.2$ & M1 & $11.3-11.5$ & $\mathrm{x}-11.4$ \\
\hline M2 & $9.8-12.1$ & $10.0-11.9$ & M2 & $10.3-10.9$ & $10.8-10.8$ \\
\hline M3 & $\mathrm{x}-\mathrm{x}$ & $\mathrm{x}-\mathrm{x}$ & $\mathrm{M} 3$ & $10.8-9.6$ & $9.9-9.8$ \\
\hline
\end{tabular}

Tabl. VIII - Caractères métriques du corps sternal.

Sternum metric characters.

\begin{tabular}{|l|l|}
\hline Longueur du corps sternal (3) & 91 \\
\hline Largeur du corps sternal (5) & 32 \\
\hline Épaisseur au milieu & 9 \\
\hline
\end{tabular}

Tabl. IX - Caractères métriques des clavicules.

Clavicle metric characters.

\begin{tabular}{|l|c|c|}
\hline & $\mathrm{D}$ & $\mathrm{G}$ \\
\hline Longueur maximale (1) & $>140$ & 155 \\
Périmètre au milieu (6) & 38 & 34 \\
Largeur externe maximale & 27 & 23 \\
Diamètre horizontal interne & - & 16 \\
Diamètre vertical interne & - & 25 \\
Diamètre horizontal au milieu (5) & 14 & 13 \\
Diamètre vertical au milieu (4) & 10 & 9 \\
& & \\
Indice de robustesse (6/1) & $<27.1$ & 21.9 \\
Indice de largeur externe & $<19.3$ & 14.8 \\
Indice diaphysaire (4/5) & 71.4 & 69.2 \\
\hline
\end{tabular}

Tabl. X - Caractères métriques des scapulas.

Scapula metrical metric characters.

\begin{tabular}{|l|c|c|}
\hline & $\mathrm{D}$ & $\mathrm{G}$ \\
\hline Hauteur (1) & - & 153 \\
Largeur (2) & - & $\# 102$ \\
Longueur du bord axillaire (3) & - & - \\
Longueur du bord crânial (4) & - & 79 \\
Hauteur proj. fosse sous-épineuse (5) & - & - \\
Hauteur anat. fosse sous-épineuse (5a) & - & - \\
Hauteur proj. fosse sus-épineuse (6) & - & - \\
Hauteur anat. fosse sus-épineuse (6a) & - & $\# 47$ \\
Longueur de l'épine scapulaire (7) & - & $\# 136$ \\
Hauteur de la cavité glénoïde (12) & $\# 40$ & 40 \\
Largeur de la cavité glénoïde (13) & 27 & 27 \\
Angle axillo-spinal (16) & - & $\# 55^{\circ}$ \\
Indice scapulaire (2/1) & - & - \\
Indice glénoïdien (13/12) & $\# 67.5$ & 67.5 \\
\hline
\end{tabular}

Tabl. XI - Caractères métriques des humérus.

Humerus metric characters.

\begin{tabular}{|l|c|c|}
\hline & $\mathrm{D}$ & $\mathrm{G}$ \\
\hline Longueur maximale (1) & $>320$ & 322 \\
Longueur physiologique (2) & - & 319 \\
Périmètre minimal (7) & 67.5 & 65 \\
Périmètre au milieu (7a) & 70 & 68 \\
Diamètre maximal au milieu (5) & 24 & 23 \\
Diamètre minimal au milieu (6) & 18 & 18 \\
Largeur de l'épiphyse distale (4) & - & 64 \\
Diamètre transversal de la tête (9) & - & - \\
Diamètre sagittal de la tête (10) & - & 48 \\
& & \\
Angle d'inclinaison & - & - \\
Angle de déclinaison & - & \\
& & 21.1 \\
Indice de robustesse (7/1) & $<21.1$ & 78.3 \\
Indice de robustesse au milieu (7a/1) & $<21.9$ & - \\
Indice diaphysaire (6/5) & 75.0 & \\
Indice de la tête (9/10) & - & \\
\end{tabular}

Tabl. XII - Caractères métriques des radius.

Radius metric characters.

\begin{tabular}{|l|c|c|}
\hline & $\mathrm{D}$ & $\mathrm{G}$ \\
\hline Longueur maximale (1) & - & $\# 250$ \\
Longueur physiologique (2) & - & 238 \\
Périmètre minimal (3) & 42.5 & 42.5 \\
Diam. transverse maximal diaphyse (4) & - & 14 \\
Diam. sagittal diaphyse (5) & - & 12.5 \\
Périmètre de la tête (5(3)) & - & 70 \\
Périmètre du col (5(4)) & 46 & 46 \\
Hauteur maximale de la tête & - & 8 \\
Hauteur minimale de la tête & - & 4 \\
Diam. transversal épiph. distale (5(6)) & - & - \\
Diam. sagittal épiph. distale & - & - \\
& & $\# 175^{\circ}$ \\
Angle collo-diaphysaire (7) & $\# 173^{\circ}$ & \\
Indice de robustesse (3/1) & - & 89.3 \\
Indice diaphysaire & - & \\
\hline
\end{tabular}


Tabl. XIII - Caractères métriques des ulnas.

Ulna metric characters.

\begin{tabular}{|l|c|c|}
\hline & $\mathrm{D}$ & $\mathrm{G}$ \\
\hline Longueur maximale (1) & - & $\# 272$ \\
Longueur physiologique (2) & - & 242 \\
Périmètre minimal (3) & - & 34 \\
Diam. sous-sigm. transversal (13) & 25 & 26 \\
Diam. sous-sigm. sagittal (14) & - & 24 \\
Diam. transverse maximal diaphyse (12) & - & 15 \\
Diam. sagittal diaphyse (11) & - & 12.5 \\
Hauteur échancrure sigmoïde & 12 & - \\
Diam. max. épiphyse distale & - & 14.5 \\
Diam. min. épiphyse distale & - & 12.0 \\
& - & 83.3 \\
\hline Indice de robustesse (3/1) & - & 12.5 \\
Indice de robustesse (3/2) & - & 14.0 \\
Indice de platôlénie (13/14) & - & \\
Indice diaphysaire (11/12) & - & \\
& - & \\
\hline
\end{tabular}

Tabl. XIV - Caractères métriques des fémurs. Femur metric characters.

\begin{tabular}{|l|c|c|}
\hline & $\mathrm{D}$ & $\mathrm{G}$ \\
\hline Longueur en position (2) & $>422$ & $\# 447$ \\
Longueur maximale (1) & $>422$ & $\# 449$ \\
Longueur au grand trochanter (3) & $>407$ & $\# 434$ \\
Périmètre au milieu (8) & 91 & 92 \\
Périmètre du col (17) & 100 & 101 \\
Périmètre de la tête (20) & $>148$ & $>140$ \\
Diamètre sagittal au milieu (6) & 30 & 30 \\
Diamètre transversal au milieu (7) & 28.5 & 30 \\
Diamètre sagittal sous-troch. (10) & 23 & 23 \\
Diamètre transversal sous-troch. (9) & 34 & 34 \\
Diamètre vertical de la tête (18) & 48 & 46 \\
Diamètre horizontal de la tête (19) & 47 & 46 \\
Largeur bicondylienne (21) & - & - \\
Longueur du condyle interne (24) & - & - \\
Longueur du condyle externe (23) & - & - \\
& & \\
Angle d'inclinaison (29) & $\# 120^{\circ}$ & $\# 124^{\circ}$ \\
Angle de torsion (28) & - & - \\
Angle de divergence (30) & - & - \\
Indice de robustesse (8/2) & -21.6 & $\# 20.6$ \\
Indice de robustesse (6+7/2) & 105.3 & 100.0 \\
Indice de platymétrie (10/9) & 97.9 & 100.0 \\
Indice pilastrique (6/7) & & \\
Indice de la tête (19/18) & & \\
& -13.4 \\
\hline
\end{tabular}

Tabl. XV - Caractères métriques des rotules.

Patella metric characters.

\begin{tabular}{|l|c|c|}
\hline & $\mathrm{D}$ & $\mathrm{G}$ \\
\hline Hauteur maximale (1) & $>41$ & $>39$ \\
Largeur maximale (2) & 40.5 & 40.5 \\
Épaisseur (3) & 19 & 19.5 \\
\hline
\end{tabular}

Tabl. XVI - Caractères métriques des tibias.

Tibia metric characters.

\begin{tabular}{|l|c|c|}
\hline & $\mathrm{D}$ & $\mathrm{G}$ \\
\hline Longueur (1) & $\# 375$ & - \\
Longueur condylo-talienne (2) & - & - \\
Périmètre minimal (10b) & 79 & 80 \\
Diam. transversal au trou nourricier (9a) & 26 & 26 \\
Diam. sagittal au trou nourricier (8a) & 36.5 & 36.5 \\
Diam. transversal au milieu (9) & 24 & 23.5 \\
Diam. sagittal au milieu (8) & 33 & 32.5 \\
Diam. transversal de l'épiph. prox. (3) & - & - \\
Diam. sagittal de l'épiph. prox. (4) & - & - \\
Diam. transversal de l'épiph. dist. (6) & - & - \\
Diam. sagittal de l'épiph. dist. (7) & - & - \\
& & \\
Angle de divergence & - & - \\
Angle de rétroversion (12) & - & - \\
Angle d'inclinaison (13) & - & - \\
& & 71.2 \\
Indice de robustesse (10b/1) & $\# 21.1$ & 72.3 \\
Indice cnémique (9a/8a) & 71.2 & \\
Indice diaphysaire (9/8) & 72.7 & \\
\hline
\end{tabular}

Tabl. XVII - Caractères métriques des fibulas.

Fibula metric characters.

\begin{tabular}{|l|c|c|}
\hline & $\mathrm{D}$ & $\mathrm{G}$ \\
\hline Longueur (1) & $\# 375$ & - \\
Longueur condylo-talienne (2) & - & - \\
Périmètre minimal (10b) & 79 & 80 \\
Diam. transversal au trou nourricier (9a) & 26 & 26 \\
Diam. sagittal au trou nourricier (8a) & 36.5 & 36.5 \\
Diam. transversal au milieu (9) & 24 & 23.5 \\
Diam. sagittal au milieu (8) & 33 & 32.5 \\
Diam. transversal de l'épiph. prox. (3) & - & - \\
Diam. sagittal de l'épiph. prox. (4) & - & - \\
Diam. transversal de l'épiph. dist. (6) & - & - \\
Diam. sagittal de l'épiph. dist. (7) & - & - \\
Angle de divergence & & \\
Angle de rétroversion (12) & - & - \\
Angle d'inclinaison (13) & - & - \\
& - & - \\
Indice de robustesse (10b/1) & \# 21.1 & - \\
Indice cnémique (9a/8a) & 71.2 & 71.2 \\
Indice diaphysaire (9/8) & 72.7 & 72.3 \\
\hline
\end{tabular}


Tabl. XVIII - Caractères métriques des talus.

Talus metric characters.

\begin{tabular}{|c|c|c|}
\hline & $\mathrm{D}$ & G \\
\hline Longueur du talus (1) & - & 52 \\
\hline Longueur maximale (1a) & - & 57.5 \\
\hline Longueur totale (1b) & 55 & 56 \\
\hline Largeur du talus (2) & - & 43 \\
\hline Largeur du talus (2a) & - & \# 42 \\
\hline Largeur des 3 facettes art. (2b) & - & 45 \\
\hline Hauteur du talus (3) & - & 31 \\
\hline Hauteur du talus (3(1)) & - & 32.5 \\
\hline Longueur de la trochlée (4) & 36 & 37 \\
\hline Largeur de la trochlée (5) & - & 31 \\
\hline Largeur port. de la trochlée (5(1)) & - & \# 22 \\
\hline Largeur ant. de la trochlée (5(2)) & 31 & \# 31 \\
\hline Longueur tête et col (8) & - & 19 \\
\hline Longueur du caput tali (9) & - & 35 \\
\hline Largeur du caput tali (10) & - & 24 \\
\hline Longueur face art. calc. dorsale (12) & 32 & 34 \\
\hline Largeur face art. calc. dorsale (13) & - & 24 \\
\hline Profondeur face art. calc. dorsale (14) & 5.5 & 5.5 \\
\hline Angle de déviation face art. calc. dors. (15) & - & $51^{\circ}$ \\
\hline Angle de déviation du col (16) & - & $18^{\circ}$ \\
\hline Angle de torsion de la tête (17) & - & $\# 51^{\circ}$ \\
\hline Angle de torsion de la tête (17a) & - & $\# 43^{\circ}$ \\
\hline
\end{tabular}

Tabl. XIX - Caractères métriques des naviculaires.

Navicular metric characters.

\begin{tabular}{|l|c|c|}
\hline & D & G \\
\hline Largeur (1) & - & $>36$ \\
Hauteur (2) & 29 & 29 \\
Longueur max. face art. prox. (3) & - & $\# 30$ \\
Largeur face art. prox. (4) & - & 26 \\
Profondeur face art. prox. (5) & - & 5 \\
Largeur max. face art. cunéiformes (6) & - & 36 \\
Epaisseur minimale (7) & 9 & 9 \\
Epaisseur maximale (8) & - & 18.5 \\
& & \\
\hline
\end{tabular}

Tabl. XX - Caractères métriques des premiers cunéiformes.

\section{First cuneiform metric characters.}

\begin{tabular}{|c|c|c|}
\hline & $\mathrm{D}$ & G \\
\hline Longueur inférieure (1) & 25 & 25 \\
\hline Longueur médiane (2) & 23 & 23.5 \\
\hline Longueur supérieure (3) & 25.5 & 25 \\
\hline Hauteur surf. art. prox. (4) & 21.5 & 21.5 \\
\hline Hauteur surf. art. dist. (5) & 26.5 & - \\
\hline Hauteur proximale (6) & 24 & 24 \\
\hline Hauteur distale (7) & 28.5 & - \\
\hline
\end{tabular}

Tabl. XXI - Caractères métriques des deuxièmes et troisièmes cunéiformes.

Second and third cuneiform metric characters.

\begin{tabular}{|l|l|l|l|l|}
\hline Rang Côté & II D & II G & III D & III G \\
\hline & & & & \\
Longueur supérieure (1) & 17.5 & 16.5 & 23.5 & 22 \\
Largeur médiane supérieure (2) & 16 & 15.5 & 16 & 16 \\
Largeur distale (3) & $(13)$ & $(12.5)$ & 14 & 14.5 \\
Largeur proximale (4) & $(17)$ & $(17)$ & 13 & 12.5 \\
& & & & \\
\hline
\end{tabular}

Tabl. XXII - Caractères métriques des métatarsiens.

Metatarsal metric characters.

\begin{tabular}{|l|c|c|c|c|c|c|c|c|c|c|}
\hline Rayon Côté & I D & I G & II D & II G & III D & III G & IV D & IV G & V D & V G \\
\hline Longueur maximale (1) & 59.5 & 60 & 75 & 76 & 70 & 70.5 & - & $\# 70$ & 69.5 & - \\
Longueur (1 et 2) & 58 & 59 & 73 & 73.5 & 68 & 69 & - & $\# 67$ & 63 & - \\
Largeur au milieu (3) & 12 & 12 & 8.5 & 8 & 8 & 8 & 10 & 10 & 10 & - \\
Hauteur au milieu (4) & 12 & 11.5 & 8 & 9 & 8.5 & 9.5 & 7 & 6.5 & 6.5 & - \\
Largeur base (6) & 18 & - & 15.5 & 16 & 15 & 14 & 11 & 12 & 21 & - \\
Hauteur base (7) & 28 & 29 & $>19$ & $>20$ & $>21$ & $>20$ & - & - & 15 & - \\
Largeur tête (8) & 21 & $\# 20$ & 12 & 12.5 & 11 & 12 & - & 11 & - & - \\
Hauteur tête (9) & 20 & 20 & - & 15 & - & - & - & - & - & - \\
Périmètre au milieu & 40 & 40 & 27 & 28 & 29 & 29 & 29 & 29 & 28.5 & - \\
\hline
\end{tabular}


Tabl. XXIII-Caractères métriques des phalanges de gros orteils (Ppl : phalange proximale de l'hallux, Pdl : phalange distale de I'hallux).

Metric characters of big toe phalanges (Ppl : hallux proximal phalanx, PdI : hallux distal phalanx)

\begin{tabular}{|l|c|c|c|c|}
\hline Type Côté & PpI D & PpI G & PdI D & PdI G \\
\hline & 31.5 & 32 & 26.5 & 25 \\
Longueur maximale & 28 & 27.5 & 24 & 23 \\
Longueur & 13 & 12.5 & - & - \\
Largeur au milieu & 10 & 9.5 & - & - \\
Hauteur au milieu & 19 & 19 & 19.5 & 19 \\
Largeur base & 15 & 16 & - & 10.5 \\
Hauteur base & 16 & 16 & - & - \\
Largeur tête & 10 & 10 & - & - \\
Hauteur tête & & & & \\
& &
\end{tabular}

Tabl. XXIV - Détermination de la stature à partir des grands os longs des membres.

Height determination on large long bones.

\begin{tabular}{|l|c|c|c|c|c|}
\hline Os & $\begin{array}{c}\text { Long. } \\
\text { max }\end{array}$ & $\begin{array}{c}\text { Long. } \\
\text { phys. }\end{array}$ & Manouvrier & $\begin{array}{c}\text { Trotter } \\
\text { et } \\
\text { Gleser }\end{array}$ & Olivier \\
\hline Fémur G & 449 & 447 & 167 & 168 & 167 \\
Tibia D & 375 & & 168 & 175 & 170 \\
Humérus G & 322 & & 165 & 170 & 166 \\
Ulna G & 272 & & 170 & 174.5 & 174 \\
Radius G & 250 & & 169 & 173.5 & 172 \\
Fibula G & 370 & & 168 & 171 & 170 \\
Fémur + Tibia & 824 & 822 & & 170.5 & 168.5 \\
& & & & & \\
\hline
\end{tabular}

Tabl. XXV - Présence de lésions d'hypoplasie linéaire de l'émail sur la face vestibulaire des dents (? = examen impossible en raison du tartre).

Presence of hypoplasia lesions in enamel on the vestibular side of teeth (? = examination impossible due to plaque).

\begin{tabular}{|l|c|c|c|c|}
\hline \multirow{2}{*}{} & \multicolumn{2}{|c|}{ Dents supérieures } & \multicolumn{2}{c|}{ Dents inférieures } \\
\cline { 2 - 5 } & G & D & G & D \\
\hline \multirow{2}{*}{ I1 } & + & 0 & + & + \\
I2 & + & + & + & + \\
C & ++ & ++ & ++ & ++ \\
P1 & ++ & ++ & ++ & ++ \\
P2 & ++ & + & + & + \\
M1 & - & ++ & ++ & $?$ \\
M2 & $?$ & $?$ & $?$ & $?$ \\
M3 & - & - & 0 & $?$ \\
& & & & \\
\hline
\end{tabular}




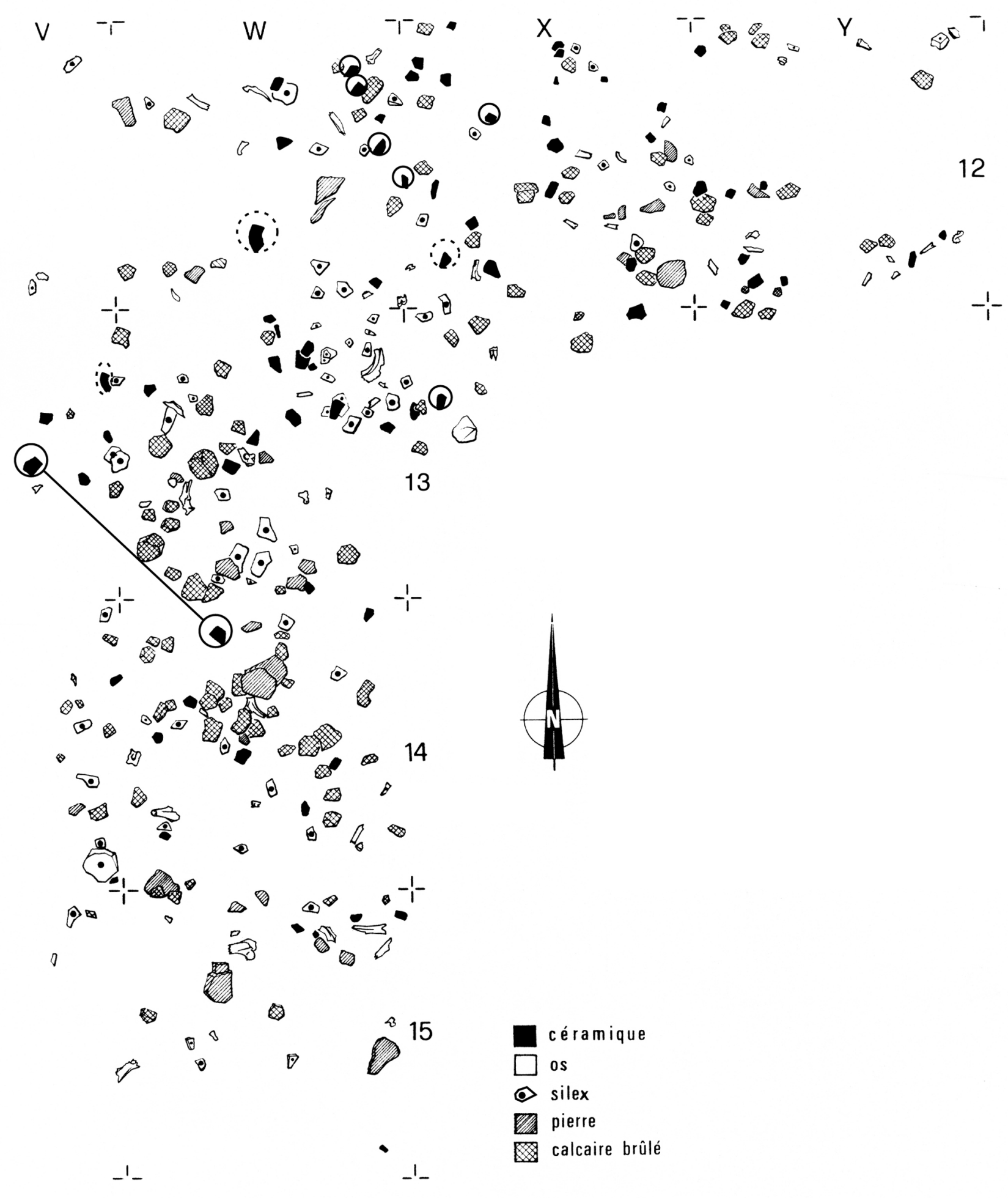

Fig. 14 - Gonesse (Val-d'Oise), La Fosse aux Larrons : plan de répartition des vestiges significatifs de la couche 2 ; entourés d'un cercle, les tessons attribuables avec plus (trait plein) ou moins de certitude (tiretés) à un petit bol,

cf. fig. 17, $n^{\circ} 4($ V $13.5+1$ ? , W 12.1, 22, 25, $28+9$ ?, W 13.2 + 40 ? , W 14.11, X 12.22, 26+6?, X 13.3).

Gonesse (Val-d'Oise), La Fosse aux Larrons: distribution plan of significant finds in layer 2; the circled sherds belong definitely (continuous line) or possibly (dotted line) to a small bowl, cf. fig. 17, $n^{\circ} 4(V 13.5+1$ ?, W 12.1, 22, 25, $28+9$ ?, W $13.2+40$ ?, W $14.11, X$ 12.22, $26+6$ ?, $X$ 13.3). 


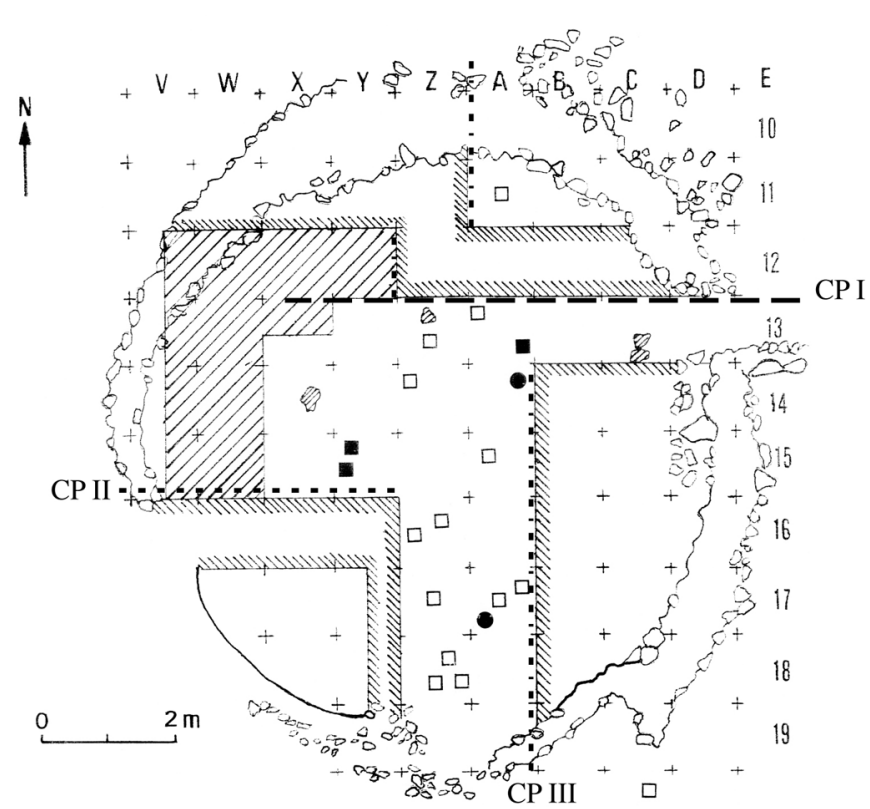

Fig. 15 - Gonesse (Val-d'Oise), La Fosse aux Larrons : plan schématique montrant la faible extension des vestiges significatifs enregistrés en place dans la couche 2 (en hachuré, la zone objet du plan de répartition de la fig. 14). En tiretés, l'emplacement des trois coupes CP I ouest-est, CP II est-ouest et CP III nord-sud.

Gonesse (Val-d'Oise), La Fosse aux Larrons: schematic plan showing the small extent of significant finds whose exact position was recorded in layer 2 (hatching is the zone shown in the distribution plan fig. 14). Dotted lines indicate the position of the three sections CP I west-east, CP II east-west and CP III north-south.

\section{LES VESTIGES MOBILIERS}

Ils ont été collectés selon les horizons stratigraphiques successivement rencontrés et coordonnés de manière adaptée à l'intérêt qu'ils paraissaient présenter ; toutefois, l'analyse géoarchéologique ultérieure a nécessité une numérotation différente du marquage initialement utilisé dont on trouvera ci-après la correspondance. Ont ainsi été distingués :

- les objets provenant du limon recouvrant la chape de pierre (couche 6) ou inclus dans les blocs la constituant (couche 5), localisés au $\mathrm{m}^{2}$ et marqués « $\mathrm{ch} »$,

- ceux issus du noyau central du tumulus subdivisé de haut en bas en couche 4 (marqués B I ou b 1), limon brun gris remanié (localisation au $\mathrm{m}^{2}$ suivi ou non de I, parfois numérotés individuellement) et en couche 3 (marqués B II ou b 2), limon brun-roux (localisés au $\mathrm{m}^{2}$ suivi de II, parfois aussi numérotés),

- ceux provenant du limon gris à la surface du tuf, interprétés comme résultant d'une couche d'occupation 2 (marqués B III ou b 3) et repérés au $\mathrm{m}^{2}$ (V à Y 12, $\mathrm{V}$ à $\mathrm{X} 13, \mathrm{~V}$ et $\mathrm{W} 14$ et 15 ), suivi de III et souvent localisés individuellement (chiffre 1 à n); s'y ajoutent les quelques pièces isolées recueillies dans les mètres $\mathrm{X}$ à $\mathrm{D} 13, \mathrm{X}$ à $\mathrm{A} 14$ et $15, \mathrm{Z}$ et $\mathrm{A} 16$ à 18 (fig. 14 et 15).

\section{LA CÉRAMIQUe}

Au total, 568 tessons ont été recueillis, 53 provenant de la chape ou du limon la recouvrant, 157 de la couche 4, 131 de la couche 3 et 227 de la couche d'occupation 2. Ce faible nombre, le peu d'éléments significatifs et l'état très médiocre des pièces n'ont pas justifié une étude de détail et nous nous bornerons à une série d'observations selon les quatre séries répertoriées en testant l'éventuelle homogénéité de l'ensemble (tabl. XXVI). Pour ces mêmes raisons, précisons que l'orientation des bords sur les illustrations reste souvent aléatoire de même que les rares restitutions proposées.

Les fragments de céramique recueillis sur et dans la chape sont petits (environ $5 \mathrm{~cm}^{2}$ ) à très petits (environ $2 \mathrm{~cm}^{2}$ ), ils ont un aspect érodé et fragmenté et sont parfois clivés dans l'épaisseur de la pâte. Malgré les réserves qu'on peut faire compte tenu des processus d'altération suite à l'enfouissement plurimillénaire et à des remaniements au moins partiels, un tri sommaire a été fait en fonction de la couleur des parois :

- 19 ont les 2 parois beiges, une surface douce et satinée, souvent craquelée avec un dégraissant à peine visible ; on relève un petit bord arrondi (fig. $16 \mathrm{n}^{\circ} 1, \mathrm{C} 10$ ) et deux possibles fragments de fond plat;

- 9 ont les 2 parois orangées, une pâte noire et le dégraissant apparaît sous forme de quelques points blancs ;

- 14 ont une paroi grise et 1 beige, externe le plus souvent, quelques grains de quartz en dégraissant;

- 9 ont les 2 parois grises, une pâte grise, un dégraissant peu apparent : deux d'entre eux ont une surface râpeuse dont l'un présente un dégraissant siliceux ;

- 2 sortent du lot : l'un, orangé clair dont la pâte et les parois montrent un abondant dégraissant coquiller parfois de forte taille ; l'autre, rouge brique, pourrait présenter un cordon imprimé très érodé ou constituer un fragment de fond : son contour très roulé interdit toute identification certaine et sa couleur pourrait évoquer un élément intrusif (fig. 16, $n^{\circ} 2, \mathrm{~W} 11$ ).

Sur ces 53 tessons, 6 ont leur plus grande longueur supérieure à $2,5 \mathrm{~cm}$, pour 22 elle est comprise entre 2 et $2,5 \mathrm{~cm}$, pour 15 entre 1,5 et $1,9 \mathrm{~cm}$ et pour 10 entre 1 et $1,4 \mathrm{~cm}$. On relève la disparité des pâtes et des cuissons et une forte altération qui se traduit par la fragmentation, l'émoussé des bords, l'aspect savonneux de certaines parois ou au contraire leur rugosité.

La céramique provenant de la couche 4 comporte des éléments de mêmes dimensions que précédemment, petits et surtout très petits, à bords émoussés tendant à des formes globulaires, ou très fragmentés.

- $\quad 77$ ont les 2 parois beiges, une surface souvent satinée ; l'un d'eux, à pâte noire, est bien cuit ; le dégraissant, rarement visible, est de la chamotte, du calcaire, parfois en association, rarement de la coquille ; on relève un fragment de fond plat, un probable bord d'assiette (fig.16, $\mathrm{n}^{\circ} 3, \mathrm{~B} 11$ ) et une languette sous un bord arrondi (fig. 16, $\mathrm{n}^{\circ} 4, \mathrm{C} 10.1$ ),

- 36 ont une paroi beige et une grise ; ils présentent souvent un dégraissant calcaire, rarement siliceux ; l'un d'eux est un bord à méplat interne et lèvre arrondie (fig. 16, $\mathrm{n}^{\circ} 5, \mathrm{X}$ 15.I),

- 25 ont les 2 parois grises ainsi que la pâte, un seul a un dégraissant coquiller, un autre la surface rugueuse ; il 


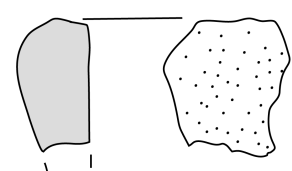

1
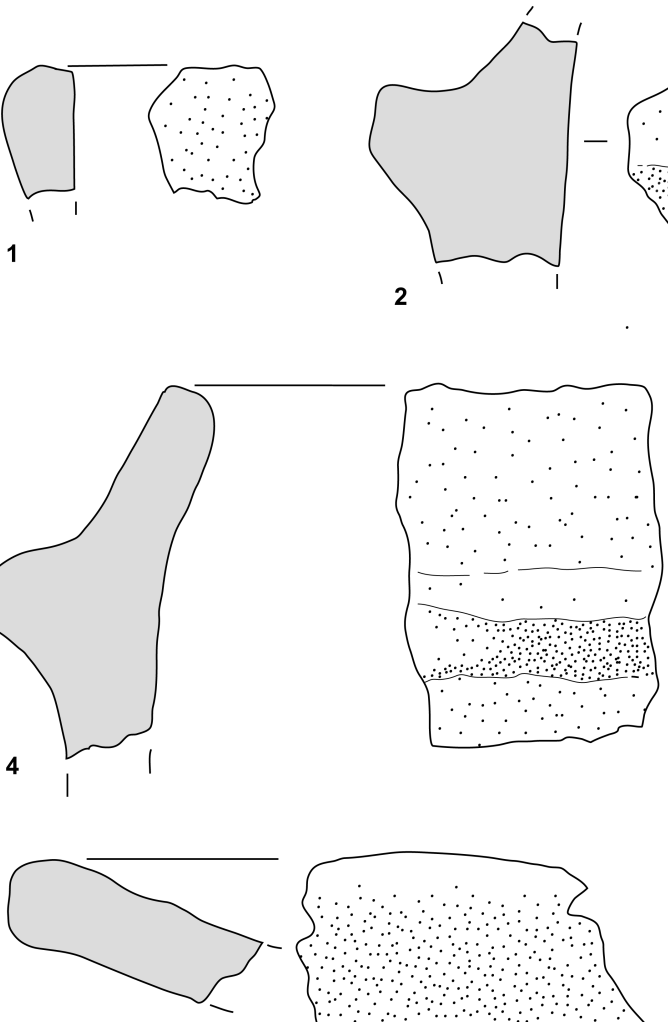

8

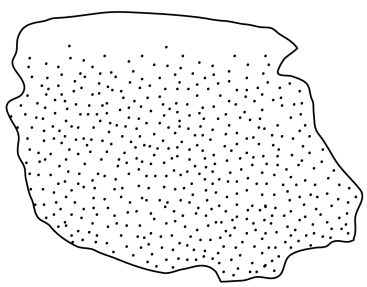

9

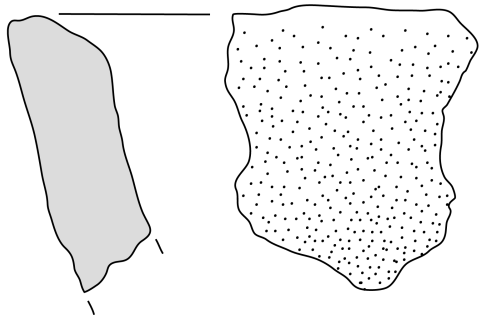

5
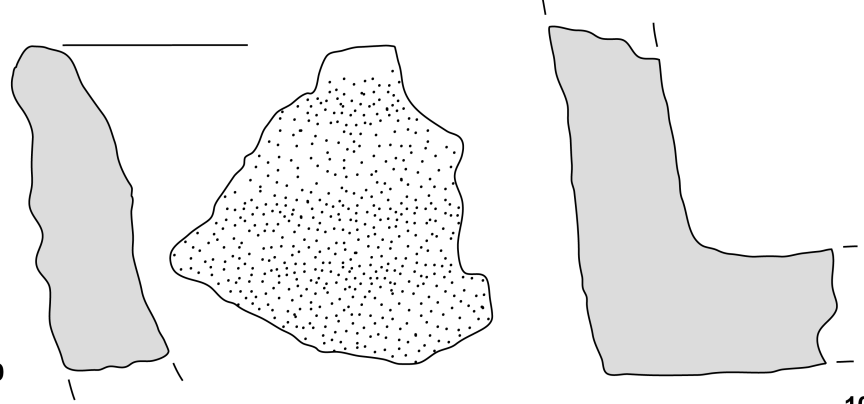

10
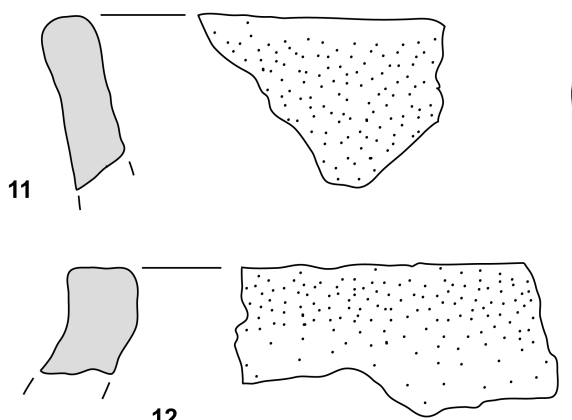

12
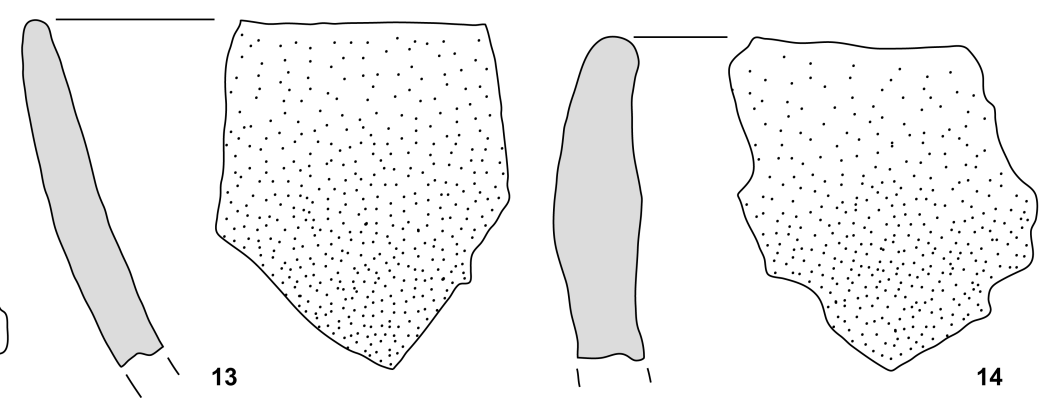

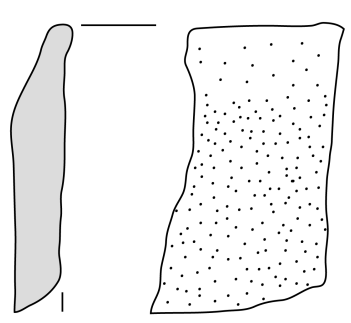

15

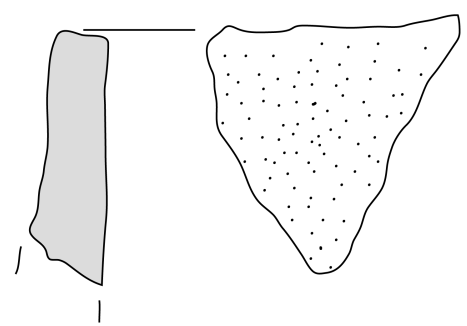

16
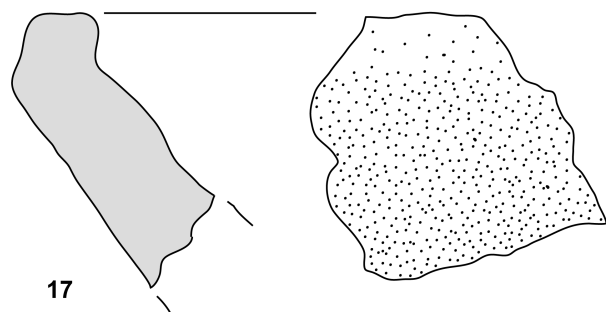

0

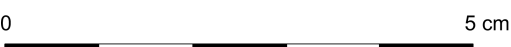

Fig. 16 - Gonesse (Val-d'Oise), La Fosse aux Larrons : fragments de bords, fonds et décors céramiques provenant de la chape et des couches 4 à $2 . n^{\circ} 1, C 10 ; n^{\circ} 2, W 11 . c h ; n^{\circ} 3, B 11 ; n^{\circ} 4, C 10.1 ; n^{\circ} 5, X 15 . I ; n^{\circ} 6, C 11 ; n^{\circ} 7, A 11$; $n^{\circ} 8, W 15 . I I ; n^{\circ} 9, W 13 . I I ; n^{\circ} 10, X 13 . I I .1 ; n^{\circ} 11, C 13 . I I ; n^{\circ} 12, Z 16 . I I ; n^{\circ} 13, Y 14 . I I .3 ; n^{\circ} 14, W 13 . I I I .31$; $n^{\circ} 15, W 14 . I I I .26 ; n^{\circ} 16, W 15 . I I I ; n^{\circ} 17, W 12 . I I I .1$.

Gonesse (Val-d'Oise), La Fosse aux Larrons: fragments of rims, bases and decorated pottery from the chape and layers 4 to 2. $n^{\circ} 1, C 10 ; n^{\circ} 2, W$ 11.ch; $n^{\circ} 3, B$ 11; $n^{\circ} 4, C 10.1 ; n^{\circ} 5, X 15 . l ; n^{\circ} 6, C 11 ; n^{\circ} 7, A 11 ; n^{\circ} 8, W 15 . I I ; n^{\circ} 9, W 13 . I I ;$ $n^{\circ} 10, X 13 . I I .1 ; n^{\circ} 11, C$ 13.II; $n^{\circ} 12, Z$ 16.II; $n^{\circ} 13, Y$ 14.II.3; $n^{\circ} 14, W$ 13.III.31; $n^{\circ} 15, W$ 14.III.26; $n^{\circ} 16, W$ 15.III; $n^{\circ} 17, W$ 12.III.1. 

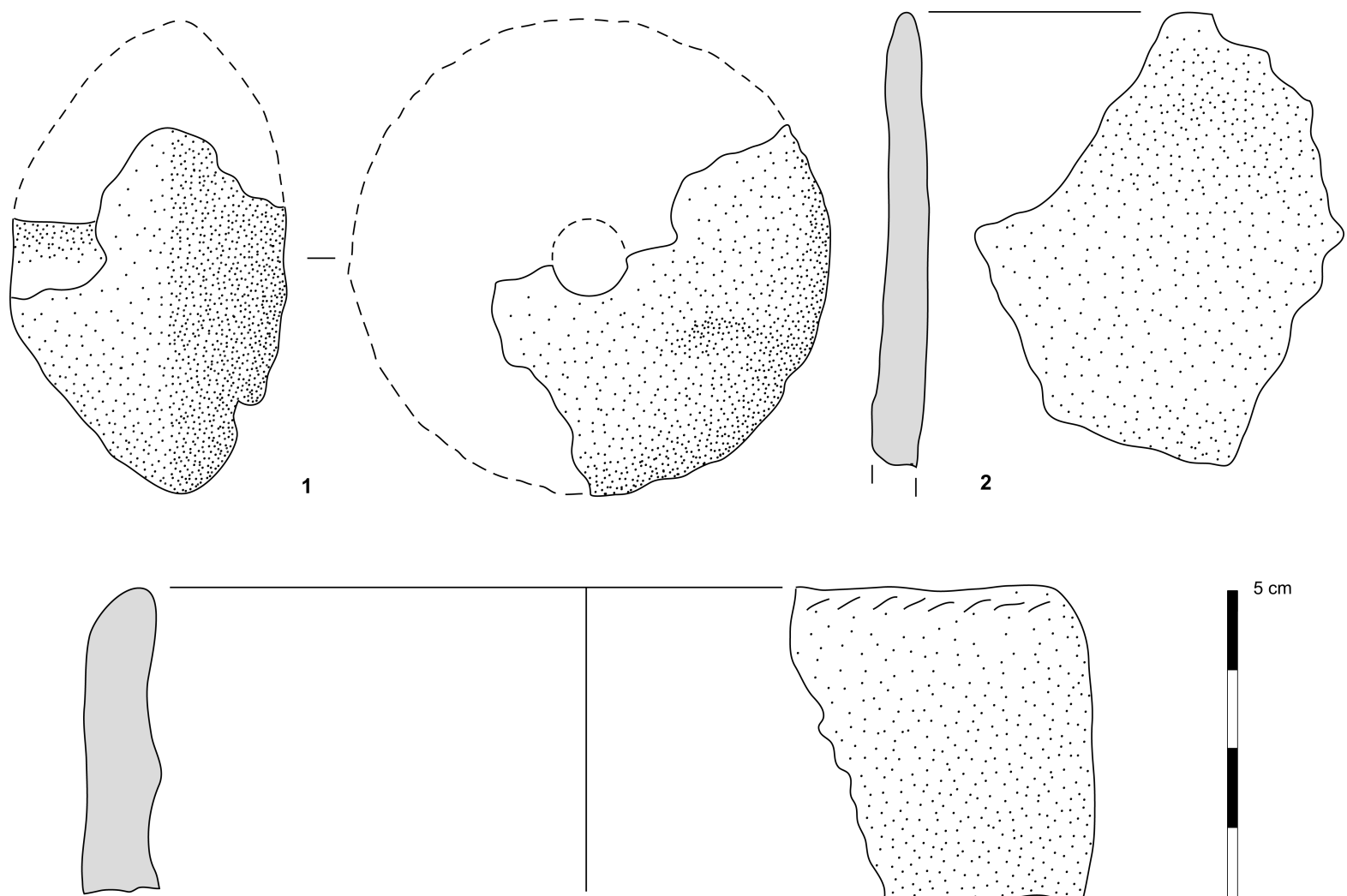

311
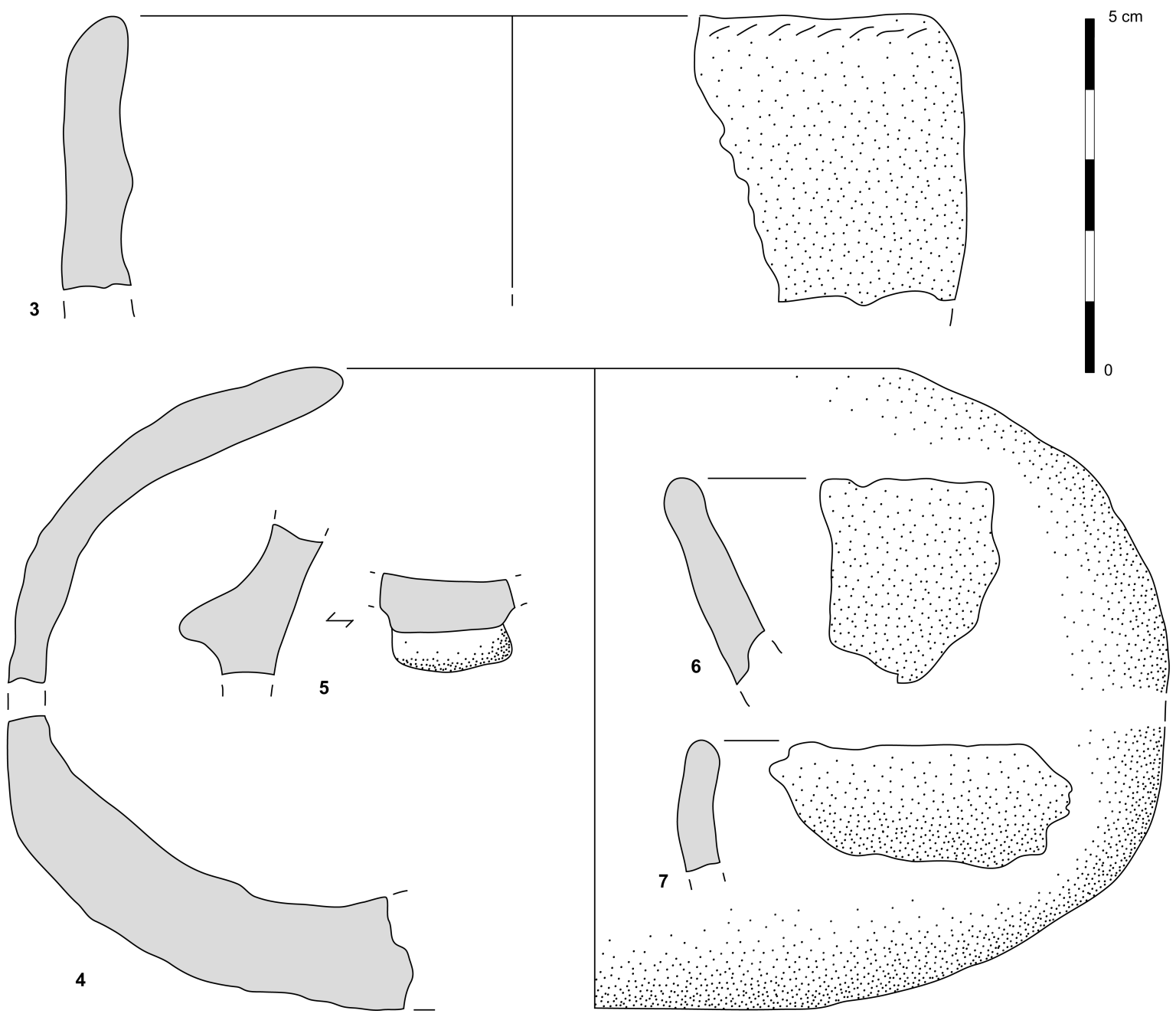

Fig. 17 - Gonesse (Val-d'Oise), La Fosse aux Larrons : fragments céramiques de la couche 2 (suite). $n^{\circ} 1, X 14 . I I I ; n^{\circ} 2, X$ $14 . I I I ; n^{\circ} 3, W 13 . I I I .8 ; n^{\circ} 4$, V 13.III.5 + W 14.III.11 + W 12.III.9; $n^{\circ} 5, X 12 . I I I .26 ; n^{\circ} 6, Y 12 . I I I .8 ; n^{\circ} 7, X 12 . I I I .21$.

Gonesse (Val-d'Oise), La Fosse aux Larrons: pottery fragments from layer 2 (continued). $n^{\circ} 1, X 14 . I I I ; n^{\circ} 2, X 14 . I I I ; n^{\circ} 3$, $W$ 13.III.8; $n^{\circ} 4$, V 13.III.5 + W 14.III.11 + W 12.III.9; $n^{\circ} 5, X 12 . I I I .26 ; n^{\circ} 6, Y$ 12.III.8; $n^{\circ} 7, X 12 . I I I .21$. 
y a au moins 2 fragments de fond plat, sinon 3 dont un bombé bien cuit (fig. 16, $\mathrm{n}^{\circ} 7, \mathrm{~A}$ 11) et un petit bord arrondi (fig. 16, $\mathrm{n}^{\circ} 6, \mathrm{C} 11$ ),

- 7 ont des parois et une pâte gris beige avec un dégraissant calcaire,

- 4 ont des parois et une pâte rougeâtre,

- 5 ont des parois et une pâte beige-orangée avec un dégraissant coquiller très abondant,

- $\quad$ enfin 3 autres sont distincts : l'un avec 1 paroi beige et l'autre orangée ; un autre avec 1 paroi orangée et l'autre noire avec une pâte à dégraissant siliceux très abondant ; un dernier avec des parois brun-rouge avec une pâte noire à dégraissant sableux.

Sur ces 157 tessons où prédomine la teinte beige, on en compte 33 dont la plus grande longueur dépasse 2,5 cm (le plus grand atteint $4,5 \mathrm{~cm}), 53$ où elle est comprise entre 2 et $2,5 \mathrm{~cm}, 50$ entre 1,5 et $1,9 \mathrm{~cm}$ et 21 entre 1 et $1,4 \mathrm{~cm}$.

La couche 3 a également livré des éléments assez petits et parfois très petits : les teintes beigeâtres sont aussi largement majoritaires ; nombreux sont les éléments roulés ou clivés dans l'épaisseur ; on relève davantage de fragments de bords.

- 73 ont les 2 parois beiges avec une surface souvent satinée et un dégraissant peu visible (deux avec des particules siliceuses) ; outre un probable fragment de fond plat figurent 3 bords dont un à forte courbure (fig. 16, $\mathrm{n}^{\circ}$ $8, \mathrm{~W} 15 . \mathrm{II}$ ) et 2 amincis (fig. 16, $\mathrm{n}^{\circ} 9, \mathrm{~W} 13 . \mathrm{II}$ ),

- 22 ont une paroi grise et l'autre beige le plus souvent externe avec une pâte où quelques grains de quartz sont parfois visibles; on relève un gros fragment de fond plat d'un diamètre de l'ordre de $13 \mathrm{~cm}$ (fig. $16, \mathrm{n}^{\circ}$ $10, \mathrm{X} 13 . \mathrm{II} .1)$ et un bord à lèvre arrondie presque plate à pâte à dégraissant de calcaire (fig. 16, n 11, C 13.II),

- 19 ont les 2 parois grises avec une pâte grise et ferme, au dégraissant peu visible; on y dénombre un probable fragment de fond plat et 4 fragments de bords dont 2 appartiennent au même récipient : l'un est un bord aminci à pâte fine et bien cuite avec de petits points de dégraissant blanc appartenant à un récipient dont le diamètre à l'ouverture pouvait être de $18 \mathrm{~cm}$ environ (fig. 16, $\mathrm{n}^{\circ} 13, \mathrm{Y}$ 14.II.3), un autre facetté à lèvre arrondie et le dernier à lèvre aplatie amorçant une courbe ou une carène (fig. $16, \mathrm{n}^{\circ} 12, \mathrm{Z}$ 16.II),

- 9 ont les 2 parois gris-beige avec une pâte épaisse et friable,

- 8 ont des parois orangées avec une pâte grise à dégraissant souvent visible de points blancs calcaires ou de chamotte ; ils peuvent être très émoussés.

Sur un total de 131 tessons, 27 ont leur plus grande longueur qui dépasse $2,5 \mathrm{~cm}$ avec quelques-uns entre 3 et $4 \mathrm{~cm}$, pour 36 elle est comprise entre 2 et $2,5 \mathrm{~cm}$, pour 45 entre 1,5 et $1,9 \mathrm{~cm}$ et pour 23 entre 1 et $1,4 \mathrm{~cm}$.

La céramique de la couche 2 (notée III) comporte également des éléments petits mais près de la moitié sont des fragments plus importants alors que la collecte a été particulièrement soignée (cf. infra) ; souvent émoussés ou très délités, certains s'effritent même au simple toucher; les tessons épais sont nombreux ; on relève plusieurs bords ainsi qu'une douzaine de tessons pouvant appartenir à un même petit bol -deux d'entre eux remontent ensemble (cf. fig. 14).
- 127 ont les deux parois beiges enserrant une pâte grisâtre comportant parfois un dégraissant de gros calibre de nature variée (calcaire surtout, coquille, siliceux) ; à côté de 3 probables fragments de fond plat, on compte un bord à lèvre arrondie rentrante (fig. 16, $\mathrm{n}^{\circ} 17$, W 12.III.1) ainsi que quatre autres dont un très fin (fig. 16, $\mathrm{n}^{\circ} 15, \mathrm{~W}$ 14.III.26), un légèrement rentrant (fig. 16, $\mathrm{n}^{\circ} 14, \mathrm{~W} 13 . \mathrm{III} .31$ ) et un plus important à incisions obliques sur la lèvre permettant de restituer un diamètre à l'ouverture d'une douzaine de centimètres (fig. 17, $\mathrm{n}^{\circ} 3, \mathrm{~W}$ 13.III.8) ; s'y ajoute un fragment de fusaïole de forme tronconique asymétrique épaisse de $3,4 \mathrm{~cm}$ pour un diamètre restitué de $6 \mathrm{~cm}$ (fig. $17, \mathrm{n}^{\circ} 1$, X 14.III),

- 16 ont les 2 parois beiges avec une pâte noire à points de dégraissant calcaires : 9 d'entre eux pourraient appartenir au même récipient dont 2 fragments de bord rentrant fortement qui remontent (fig. $17, \mathrm{n}^{\circ} 4, \mathrm{~V}$ 13.III.5 et $\mathrm{W}$ 14.III.11) ainsi que de gros fragments de fond aplati et épais (W 12.III.9) et une petite languette horizontale (fig. 17, $\mathrm{n}^{\circ}$ 5, X 12.III.26) ; deux autres fragments sont des bords probables,

- 48 ont une paroi beige-orangée et une grise interne ; 4 d'entre eux pourraient également appartenir au petit bol précédent ; on relève par ailleurs un fragment de bord à lèvre plate (fig. $16, \mathrm{n}^{\circ} 16, \mathrm{~W} 15 . \mathrm{III}$ ),

- $\quad 34$ ont les 2 parois grises avec une pâte de même teinte et un dégraissant peu visible ; on compte un fragment de fond plat et 3 bords à lèvre arrondie (fig. 17, $n^{\circ} 6, \mathrm{Y}$ 12.III.8) dont l'un pourrait appartenir à un récipient à large ouverture (plus de $20 \mathrm{~cm}$ de diamètre, fig. 17, $\mathrm{n}^{\circ}$ 7, X 12.III.21) et un autre à un haut de profil quasi rectiligne (fig. 17, $\mathrm{n}^{\circ} 2, \mathrm{X}$ 14.III),

- 2 sont des fragments d'argile, l'un assez gros fragmenté et l'autre émoussé de teinte rouge brique rappelant l'élément provenant de la chape en W 11 (cf. supra).

Sur un total de 227 tessons, 98 ont leur plus grande longueur qui dépasse $2,5 \mathrm{~cm}$, un bon nombre se situant entre 4 et $5 \mathrm{~cm}$ et deux atteignant $7,5 \mathrm{~cm}$, pour 61 elle est comprise entre 2 et $2,5 \mathrm{~cm}$, pour 51 entre 1,5 et $1,9 \mathrm{~cm}$ et pour 17 entre 1 et $1,4 \mathrm{~cm}$.

Que peut-on conclure de l'examen de ces quatre séries ? D’une manière générale, la teinte beigeâtre domine tout comme l'état d'altération très prononcée de la plupart des tessons à tel point qu'on ne peut manier certains sans qu'ils perdent de la substance : on peut penser que la cause principale en est un lieu de dépôt où le sédiment emballant a été plus ou moins constamment extrêmement humide, zone de marécages persistants avec des phases d'inondation alternant parfois avec des phases plus sèches ainsi que le montrent les analyses environnementales, malacologiques en particulier. Le tri sommaire par teinte des parois montre une proportion semblable d'une couche à l'autre, chaque lot présentant par ailleurs à tout niveau une grande hétérogénéité des récipients en céramique tout comme une grande variabilité dans la nature des dégraissants et la qualité de la cuisson des pâtes. Toutefois, la couche 2 se caractérise par un plus grand nombre d'éléments significatifs (fragments de bords ou de fonds), la présence de tessons assez volumineux et plus épais, le remontage de deux portions de bord distants de près d'un mètre ainsi que l'attribution probable de plusieurs autres tessons dispersés sur environ $2 \mathrm{~m}^{2}$ à ce même ensemble qui 

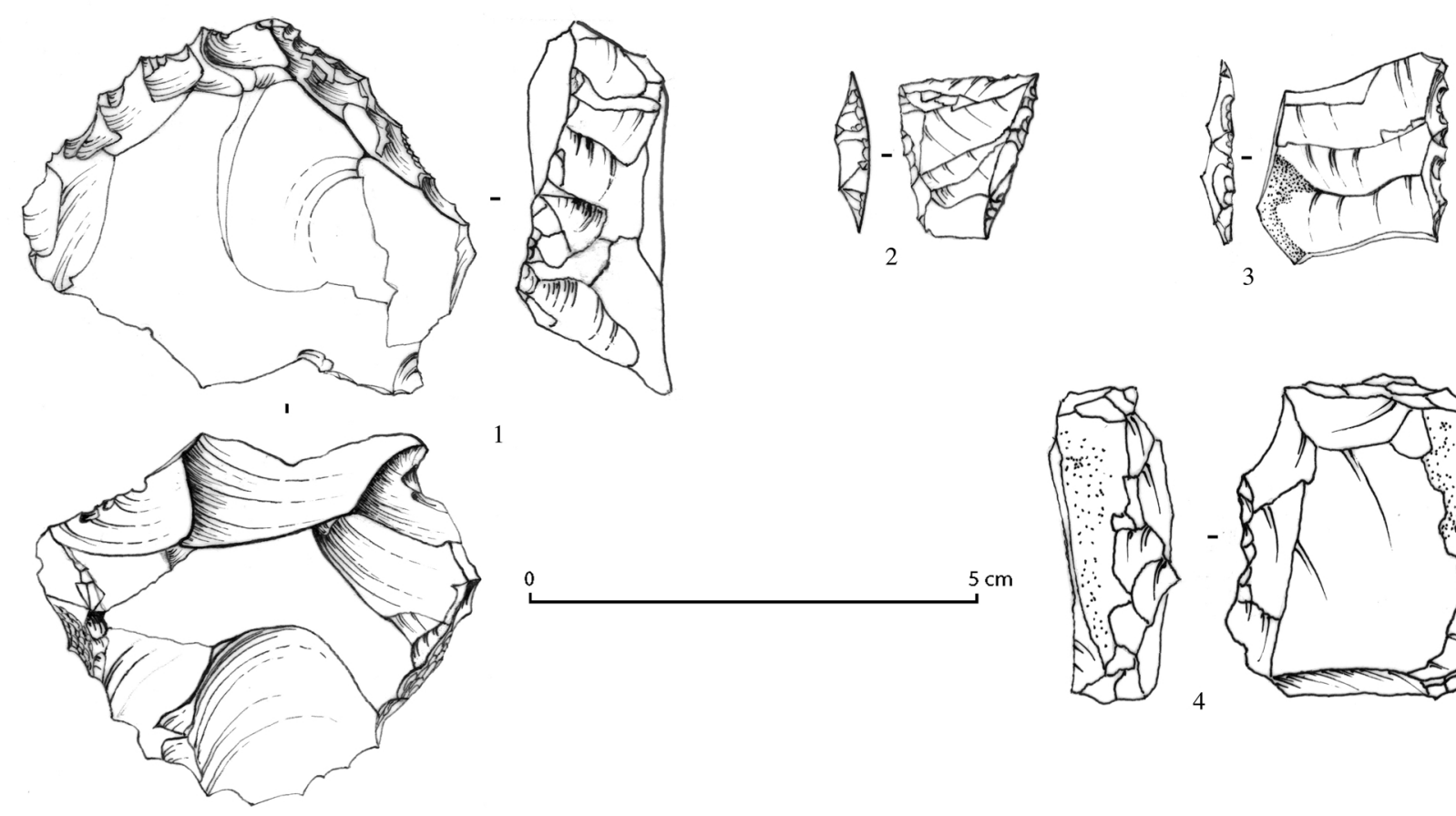

2
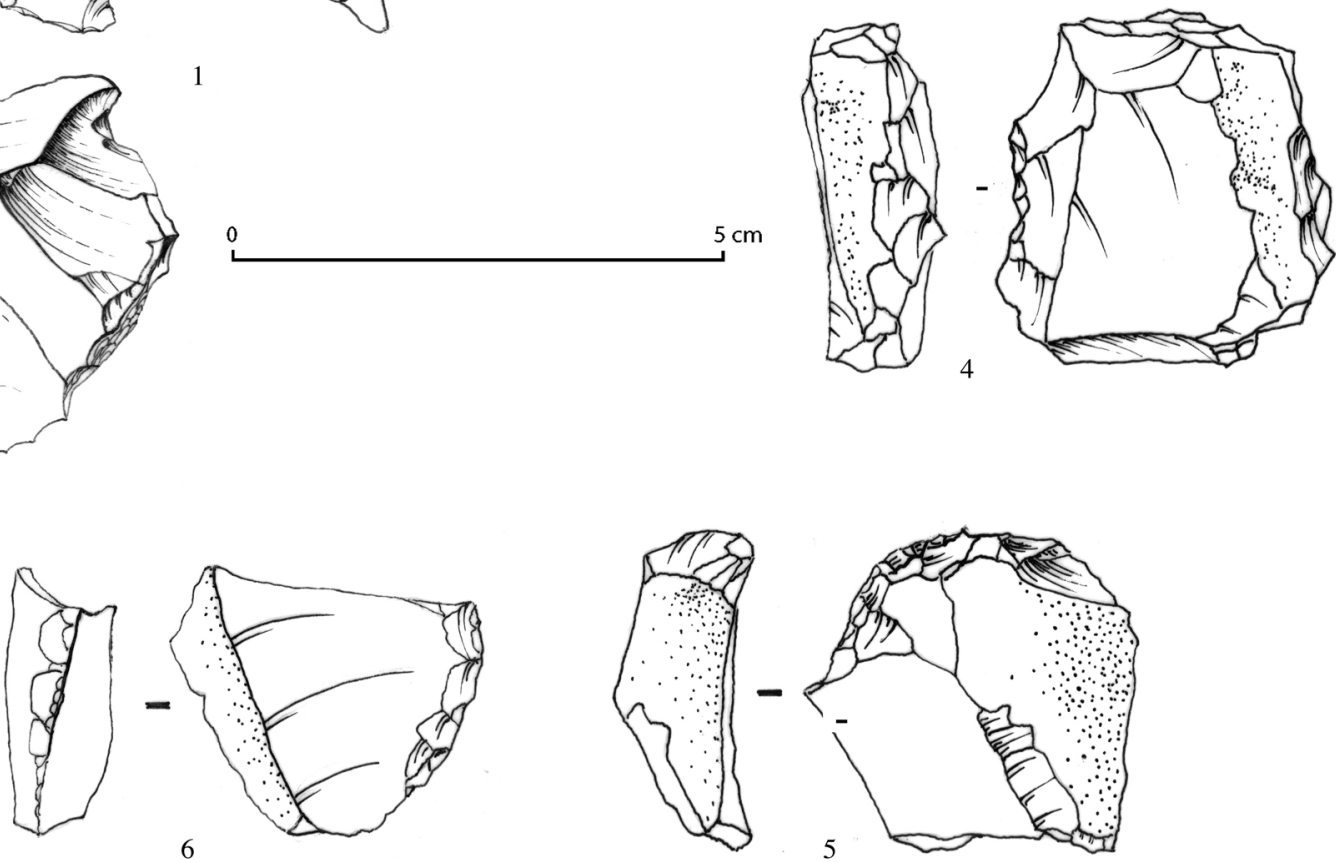

Fig. 18 - Gonesse (Val-d'Oise), La Fosse aux Larrons : industrie lithique de la chape. Armatures tranchantes : $n^{\circ} 2, C 9 \mathrm{ch} . ; n^{\circ} 3, \mathrm{C} 11 \mathrm{ch}$. Grattoirs : $n^{\circ} 1, E 13 \mathrm{ch}$; ; $n^{\circ} 4, E 17$ ch. ; $n^{\circ} 5, E 13$ ch. Racloir : $n^{\circ} 6, E 14 \mathrm{ch}$.

Gonesse (Val-d'Oise), La Fosse aux Larrons: lithic industry from the chape. Tranverse arrowheads: $n^{\circ} 2, C 9$ ch. ; $n^{\circ} 3, C 11$ ch. Scrapers: $n^{\circ}$, E 13 ch.; $n^{\circ} 4$, E 17 ch.; $n^{\circ} 5$, E 13 ch. Racloir: $n^{\circ} 6$, E 14 ch.

paraît être une sorte de petit bol à parois rentrantes qu'on dirait sommairement façonné dans une boule de pâte (fig. 17, nº).

Ainsi, même si lorsqu'on examine un ensemble de vestiges recueillis en un même lieu, on doit a priori s'interroger sur leur homogénéité et à plus forte raison lorsqu'ils proviennent de couches successives, il n'apparaît pas ici de différences significatives hormis le fait que les éléments issus de la couche de base (2) paraissent avoir été davantage protégés au sommet inégal du tuf sous-jacent que ceux emballés dans les 2 couches de limon la surmontant pour constituer le noyau du tumulus.

Quant à une éventuelle attribution chronologique, on aura constaté le peu d'éléments discrimants disponibles. La présence d'une fusaïole invite bien sûr à ne pas remonter l'ensemble avant le début du Néolithique récent : les divers fragments de fond plat, les languettes horizontales et le bord à lèvre incisée obliquement évoquent la fin de la période voire l'âge du Bronze ancien.

\section{L'INDUSTRIE LITHIQUE}

La fouille ayant été réalisée essentiellement par des personnes formées à l'étude du Paléolithique, une attention toute particulière a été portée à la collecte des produits lithiques ce qui explique, par exemple, le nombre des esquilles et cassons recueillis, tous marqués individuellement comme n'importe quelle autre pièce. Si les tranchants des silex sont vifs (hormis une armature perçante - fig. 19, $\mathrm{n}^{\circ} 4, \mathrm{D} 14.1$ dont la profonde patine blanche a arrondi l'une des faces), on observe en revanche de très fréquentes fractures dont certaines révèlent la teinte originelle du silex (par exemple, l'armature perçante fig. $19, \mathrm{n}^{\circ} 3, \mathrm{C} 10.2$ ) ce qui dénote un remaniement certain du matériel lithique tout comme, vraisemblablement, l'abondance particulière des cassons. Deux principaux types de silex ont été déterminés (expertise de F. Bostyn que nous remercions ici) : le plus abondant est une roche tertiaire brune ou beige plus ou moins foncé, fréquemment patiné en blanc parfois profondément ou en blanc bleuté plus légèrement (la teinte originelle du silex apparaissant bien sur fracture). Le second, beaucoup plus rare, est un silex secondaire crétacé de teinte bleue presque dépourvu de patine avec un simple film de cortex beige clair. Par ailleurs, de nombreux produits ont subi l'action du feu ne permettant plus une attribution précise : ils peuvent être gris plus ou moins craquelés ou à patine blanche veinée ou réticulée. 

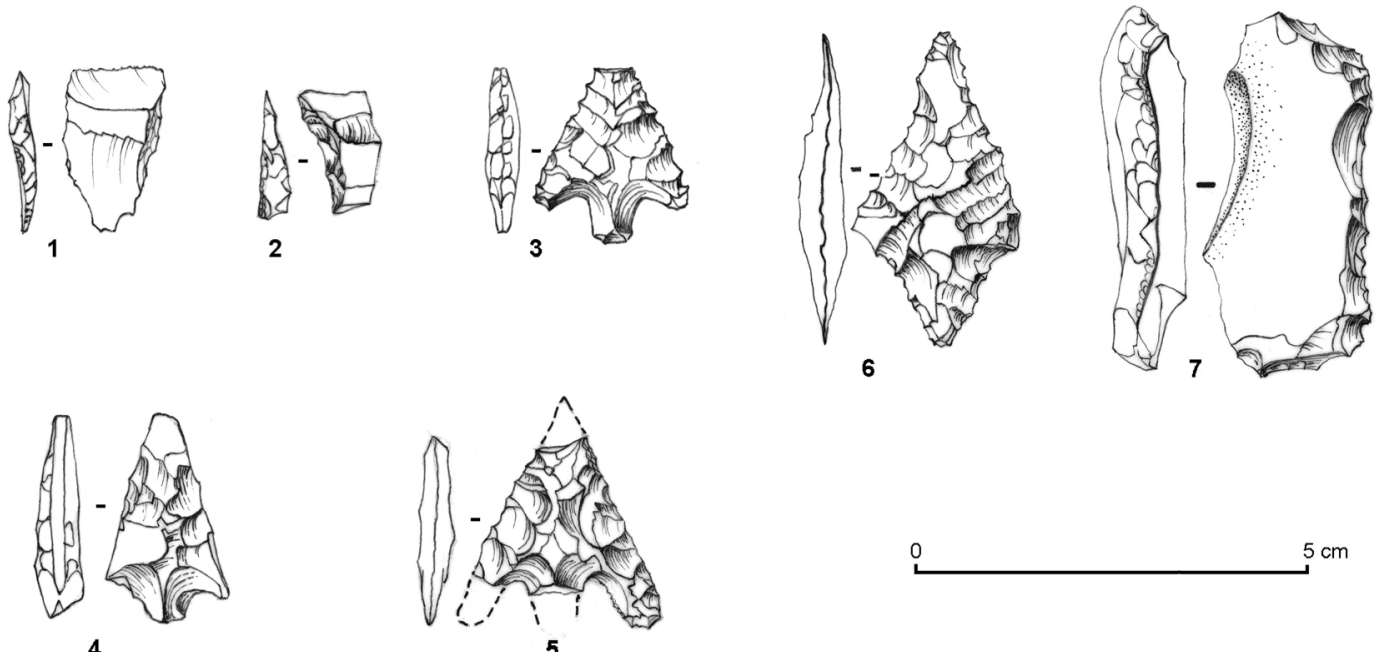

5
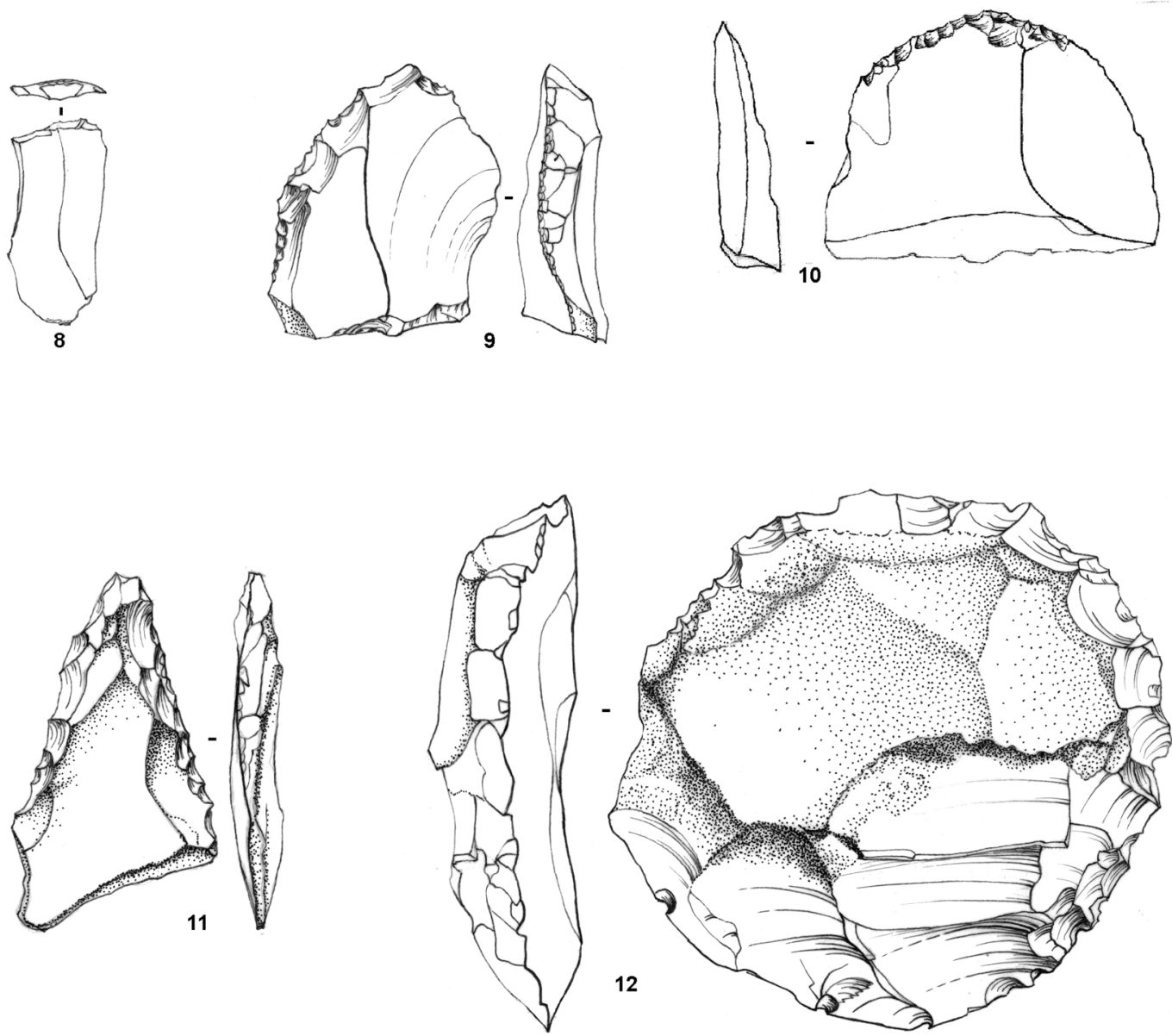

Fig. 19 - Gonesse (Val-d’Oise), La Fosse aux Larrons : industrie lithique de la couche 4 . Armatures : $n^{\circ} 1, W^{15}$ I.1; $n^{\circ} 2$, A $16 . I$; $n^{\circ} 3$, C $10.2 ; n^{\circ} 4, D 14.1 ; n^{\circ} 5, A 15.3 ; n^{\circ} 6, Y$ 12.I.1. Racloirs : $n^{\circ} 7$, A 13.I.1; $n^{\circ} 9, D$ 10.3. Pièce à troncature: $n^{\circ} 8$, W 14.I. Grattoirs : $n^{\circ} 10$, Z 15.1; $n^{\circ} 12$, F 14.1. Pointe : $n^{\circ} 11$, Z 14.7.

Gonesse (Val-d'Oise), La Fosse aux Larrons: lithic industry from layer 4. Arrowheads: $n^{\circ} 1, W^{15} I .1 ; n^{\circ} 2, A 16 . l ; n^{\circ} 3, C_{10.2 ;}$ $n^{\circ} 4, D$ 14.1; $n^{\circ} 5, A$ 15.3; $n^{\circ} 6$, Y 12.I.1. Racloirs: $n^{\circ} 7, A$ 13.I.1; $n^{\circ} 9, D$ 10.3. Truncated piece: $n^{\circ} 8, W$ 14.I.

Scrapers: $n^{\circ} 10, Z$ 15.1; $n^{\circ} 12$, F 14.1. Point: $n^{\circ} 11, Z$ 14.7. 
L'examen des produits de débitage révèle en très grande majorité un silex de médiocre qualité, à cortex et zone souscorticale fortement développés ; les produits sont globalement de petite taille, au moins aussi larges que longs, souvent épais et courts.

Seuls trois blocs nucléiformes ont été recueillis sur le niveau de base (couche 2) : deux, de dimensions similaires (4 à $5 \mathrm{~cm}$ ), ont une forme pour l'un pyramidale ne présentant que de rares enlèvements, pour l'autre d'allure plutôt globulaire avec des enlèvements plus nombreux principalement sur deux faces. Le troisième est une plaquette rectangulaire épaisse à cortex sur une face, l'autre présentant des enlèvements rasants opposés issus majoritairement des bords parallèles les plus longs.

Sur les 1535 produits recensés hors façonnage, c'est la couche 4 qui en livré le plus (652), puis la 3 (374), la 2 (285) et enfin la chape (228) : ces variations peuvent être au moins en partie simplement proportionnelles au volume de sédiment traité. En pourcentage, d'une couche à l'autre, leur composition varie peu : lames et lamelles autour de $2 \%$, éclats laminaires et fragments environ de 3 à $6 \%$, éclats et fragments environ $30 \%$ tout comme les esquilles et les cassons, catégories qui constituent chacune près d'un tiers de l'ensemble (483 esquilles et 439 cassons). Les pièces brûlées, principalement des cassons, sont nombreuses, atteignant et même dépassant $20 \%$; la couche 4 , qui rassemble près de $50 \%$ de l'ensemble des cassons, en comprend même $40 \%$.

Les produits façonnés, entiers ou fragmentaires, comptent 41 pièces.

Une brève description de chaque pièce sera donnée en respectant les couches successivement individualisées.

\section{Chape (couches 5 et 6), 6 pièces}

Armatures : les deux exemplaires sont à tranchant transversal, l'un trapézoïdal à bords abattus par retouches directes semi-abruptes sur fragment de lame ou d'éclat (C 9.ch, silex à patine blanche, $1,8 \times 1,7 \times 0,3 \mathrm{~cm}$ : fig. $18, \mathrm{n}^{\circ} 2$ ), l'autre au bord droit abattu par retouches abruptes directes et au gauche fracturé (anciennement ?) sur petit éclat (C 11.ch, silex beige à patine blanc-bleutée avec petite plage corticale, $2,2 \times 2,1 \times 0,3$ $\mathrm{cm}$ : fig. $18, \mathrm{n}^{\circ} 3$ ).

Grattoirs : trois dont un à front semi-abrupt denticulé façonné sur la face d'éclatement d'un éclat épais au talon abrasé par petites retouches répétées et à larges retouches périphériques sur la face d'enlèvement (E 13.ch, silex à patine blanc-bleutée, $4 \times 4,5 \times 1,6 \mathrm{~cm}$ : fig. $\left.18, \mathrm{n}^{\circ} 1\right)$, un autre à front sub-rectiligne obtenu par retouches semi-abruptes formant corniche sur éclat épais partiellement cortical à larges retouches d'amincissement sur la face ventrale (E 17.ch, silex à patine blanc-bleutée, $3,6 \times 3,5 \times 1,3 \mathrm{~cm}$ : fig. $18, \mathrm{n}^{\circ}$ 4) et un dernier à front abrupt par retouches directes formant corniche sur éclat court à base fracturée (E 13.ch, silex brun à patine bleutée avec plage de cortex, $3,2 \times 3,3 \times 1,2 \mathrm{~cm}$ : fig. $18, \mathrm{n}^{\circ} 5$ ).

Racloir : pièce fracturée transversalement à bord droit repris par de courtes retouches semi-abruptes directes et à bord gauche cortical (E 14.ch, silex bleuté, 2,6 x 3,1 x $1 \mathrm{~cm}$ : fig. 18, nº 6).

Couche 4 (marquée I), 14 pièces

Armatures : deux de petite taille sont à tranchant transversal, l'une trapézoïdale à bords abattus par retouches alternes (W 15.I.1, silex brun, 2 x 1,8 x 0,3 cm : fig. 19, nº), l'autre représentée par un petit fragment abattu par retouches abruptes directes en constituant probablement le bord gauche (A 16.1, silex à patine blanche, $1,6 \times 0,9 \times 0,3 \mathrm{~cm}$ : fig. $19, \mathrm{n}^{\circ} 2$ ). Les quatre autres sont perçantes : l'une a des ailerons courts bien dégagés et à extrémité anguleuse, sa pointe et l'extrémité du pédoncule sont fracturées ; elle est façonnée par retouches plates couvrantes (C 10.2, silex beige à patine bleutée, $2,2 \times 2 \times 0,4 \mathrm{~cm}$ : fig. $19, \mathrm{n}^{\circ}$ 3). Une autre possède un aileron pointu, l'autre étant fracturé comme la base du pédoncule, le bord droit est vif opposé au gauche mousse (résultat d'une altération ?) comme la pointe (D 14.1, silex brun à patine blanche profonde, 2,6 x 1,7 x 0,6 cm : fig. $\left.19, n^{\circ} 4\right)$. Une troisième dont la pointe, le pédoncule et un aileron sont fracturés conserve un aileron allongé bien dégagé à extrémité arrondie ; elle est façonnée par retouches plates bilatérales couvrantes (A 15.3, silex beige, 2,4 x 2,5 x 0,4 cm : fig. $19, \mathrm{n}^{\circ} 5$ ). La dernière est une grande armature, losangique à ailerons naissants façonnée par retouches plates bifaciales couvrantes (Y 12.I.1, silex beige à patine blanc-bleutée, 3,9 x 2,1 x $0,5 \mathrm{~cm}$ : fig. $\left.19, \mathrm{n}^{\circ} 6\right)$.

Racloirs : de morphologie très différente, l'un, sur lame épaisse, a son bord droit façonné par retouches abruptes formant corniche, la saillie du bulbe retirée par retouches plates : l'allure générale évoque les retouchoirs ou briquets malgré l'absence d'émoussé (A 13.I.1, silex gris partiellement cortical, 4,6 x 2 x $1,1 \mathrm{~cm}$ : fig. $19, \mathrm{n}^{\circ} 7$ ) ; l'autre, sur éclat, a son bord gauche aménagé par retouches directes semi-abruptes opposé à un bord vif à droite (D.10.3, silex gris blanc réticulé -brûlé-, 4 x 3,2 x $1,3 \mathrm{~cm}$ : fig. $\left.19, \mathrm{n}^{\circ} 9\right)$.

Pièce à troncature : c'est une petite lame mince à troncature oblique convexe au bord gauche fracturé sur une moitié - coup de burin improbable - (W 14.I, silex blanc-bleuté, 3,1 x 1,5 x $0,25 \mathrm{~cm}$ : fig. $\left.19, \mathrm{n}^{\circ} 8\right)$.

Grattoirs : deux pièces très différentes, l'une présentant un front de grattoir mince aménagé par courtes retouches obliques sur fragment d'éclat gélif $(\mathrm{Z} 15.1$, silex beige à patine bleutée, $3,6 \times 4,8 \times 1 \mathrm{~cm}$ : fig. $19, \mathrm{n}^{\circ} 10$ ), l'autre sur gros éclat gélif, partiellement cortical, ayant un front circulaire denticulé façonné par retouches obliques sur les trois-quarts du périmètre $(\mathrm{F} \mathrm{14.1}$, silex beige légèrement bleuté, 8 x $78 \mathrm{x}$ $1,8 \mathrm{~cm}$ : fig. $\left.19, \mathrm{n}^{\circ} 12\right)$.

Pointe : asymétrique, la surface dorsale corticale est façonnée par une courte retouche abrupte à semi-abrupte des deux bords, la face inverse montre des traces de chocs thermiques à proximité de la pointe fracturée $(\mathrm{Z}$ 14.7, silex bleuté, 5,3 × $3,1 \times 0,7 \mathrm{~cm}$ : fig. $\left.19, \mathrm{n}^{\circ} 11\right)$.

Pièces polies : deux petits éléments en attestent la présence, l'un est un petit fragment d'une face convexe en silex bleu sombre brûlé (B 11, 3,4 x 1,7 x 0,9 cm), l'autre un petit éclat en silex gris clair (X 12.I, 1,7 x 1,6 x 0,3 cm).

\section{Couche 3 (marquée II), 13 pièces}

Armatures : parmi les trois exemplaires à tranchant transversal, de forme trapézoïdale sur fragment de lame, deux sont de petite taille avec un bord fracturé et l'autre abattu par retouches directes abruptes, à gauche dans un cas (A 16.II, silex à patine blanc-bleutée, $1,5 \times 1,4 \times 0,3 \mathrm{~cm}$ : fig. $\left.20, \mathrm{n}^{\circ} 1\right)$, à droite dans l'autre (X 15.II, silex brun à patine blanche : fig. 20, $\mathrm{n}^{\circ}$ 2) ; la troisième plus grande, sans doute sur éclat, a ses deux bords abattus par retouches abruptes directes (X 13.II, silex brun à patine blanc-bleutée, $2,2 \times 23 \times 0,4 \mathrm{~cm}$ : fig. $20, \mathrm{n}^{\circ} 3$ ). La quatrième est une petite armature perçante asymétrique à ailerons pointus et à pédoncule dégagé par retouches bifaciales partielles rasantes à obliques, la pointe et une partie du bord 

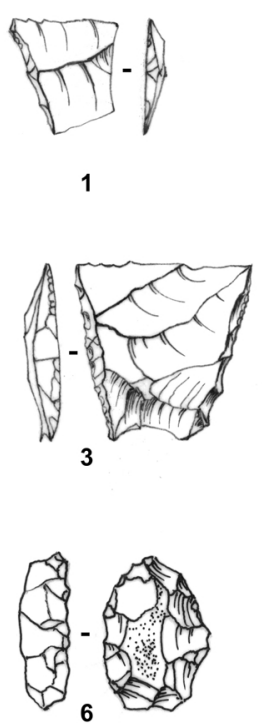

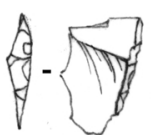

2

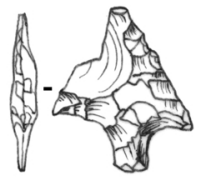

4
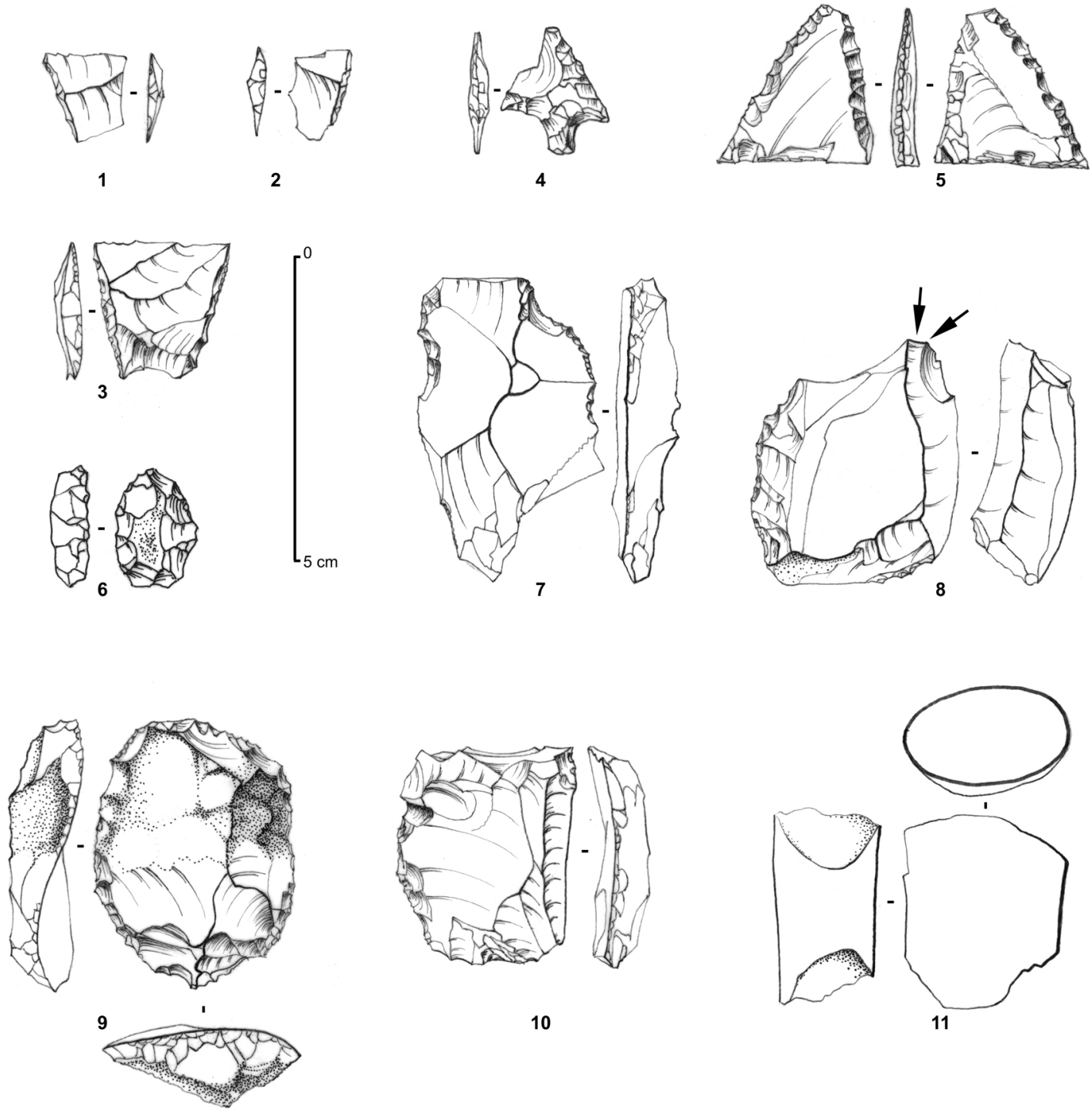

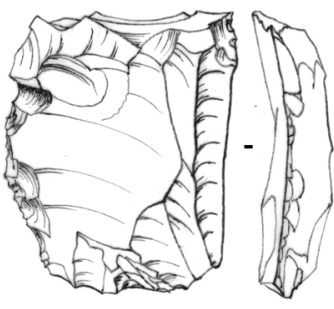

10
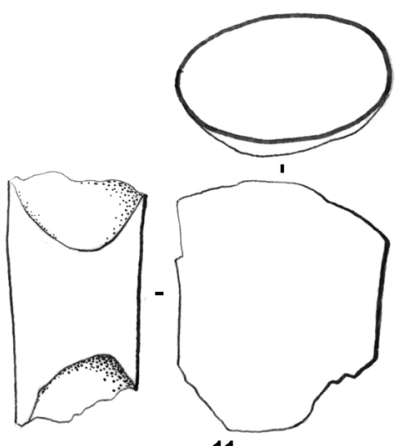
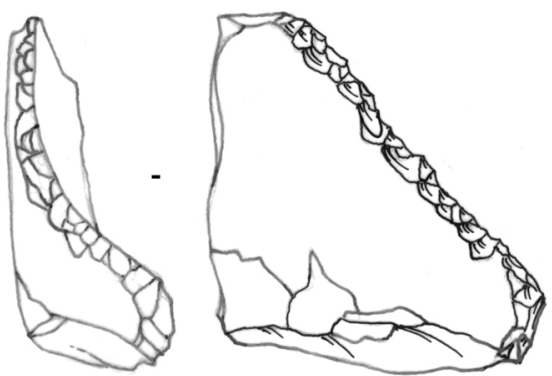

12

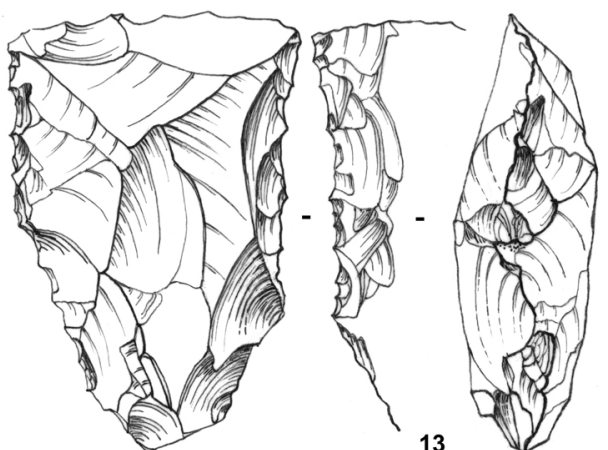

Fig. 20 - Gonesse (Val-d'Oise), La Fosse aux Larrons : industrie lithique de la couche 3. Armatures : $n^{\circ} 1$, A 16.II ; $n^{\circ} 2, X 15 . I I ; n^{\circ} 3, X 13 . I I ; n^{\circ} 4, W$ 13.II.1. Pièce foliacée : $n^{\circ} 5$, Z 15.II.10. Grattoirs : $n^{\circ} 7, X 15 . I I ; n^{\circ} 9$, Z 13.II.1. Racloirs : $n^{\circ} 6$, A 13.II ; $n^{\circ} 10$, A 14.II ; $n^{\circ} 12, Y$ Y 14.II. Burin : $n^{\circ} 8, W 15 . I I$. Tranchet : $n^{\circ} 13, X 13$ II.2. Pièce polie : $n^{\circ} 11, Y$ 15.II.6.

Gonesse (Val-d'Oise), La Fosse aux Larrons: lithic industry from layer 3. Arrowheads: $n^{\circ} 1$, A $16 . I I ; n^{\circ} 2, X 15 . I I ;$ $n^{\circ} 3, X$ 13.II; $n^{\circ} 4, W$ 13.II.1. Leaf-shaped piece: $n^{\circ}$ 5, Z 15.II.10. Scrapers: $n^{\circ} 7, X$ 15.II ; $n^{\circ}$ 9, Z 13.II.1. Racloirs: $n^{\circ} 6$, A 13.I ; $n^{\circ} 10$, A 14.II; $n^{\circ} 12$, Y 14.II. Burin: $n^{\circ} 8$, W 15.II. Tranchet: $n^{\circ} 13$, X 13 II.2. Polished piece: $n^{\circ} 11$, Y 15.II.6. 

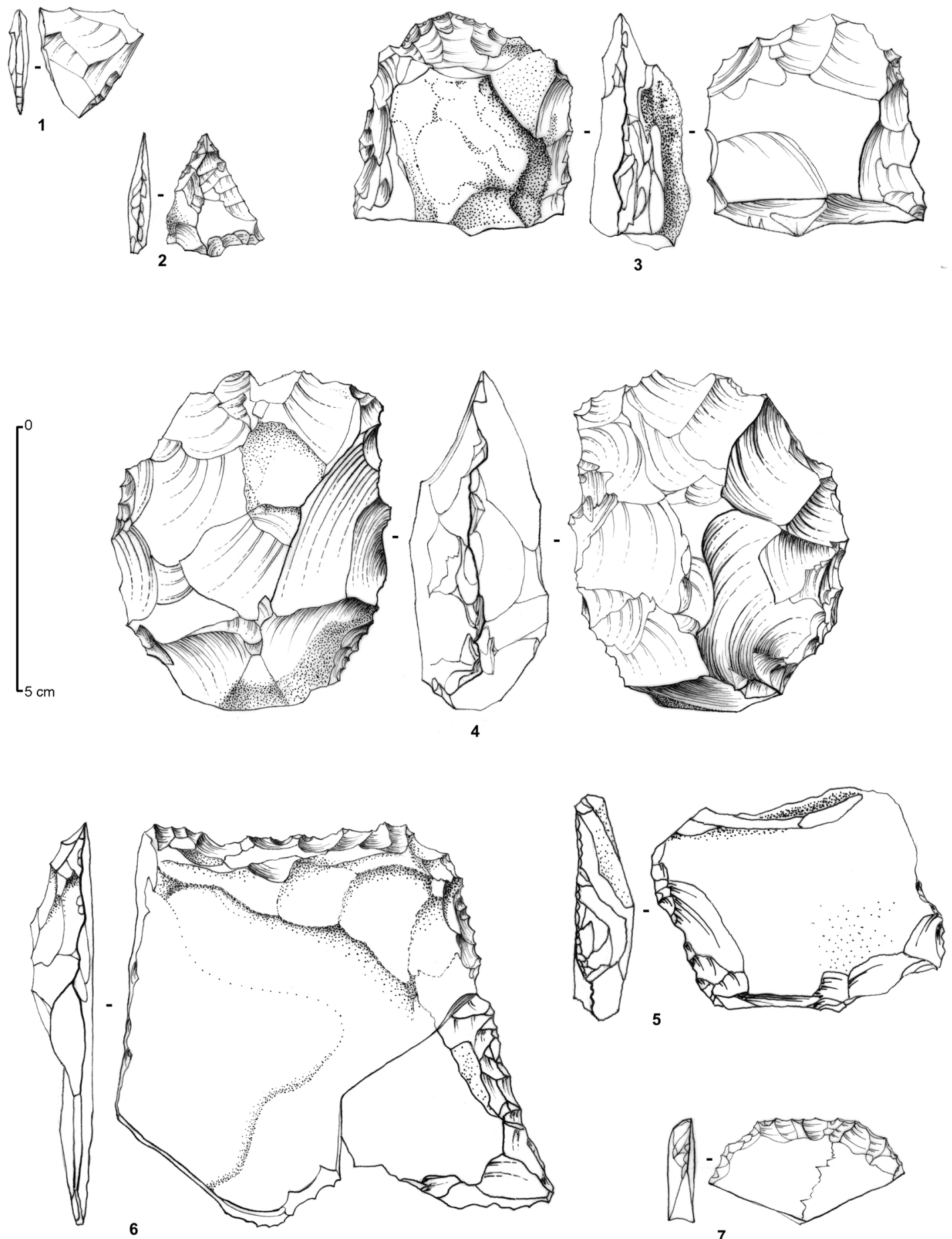

5

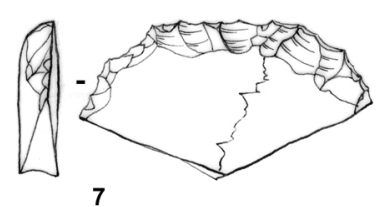

Fig. 21 - Gonesse (Val-d’Oise), La Fosse aux Larrons : industrie lithique de la couche 2. Armatures : $n^{\circ} 1, W$ 12.III.6 ; $n^{\circ} 2$, Z 17.III.1. Grattoirs : $n^{\circ} 3$, Z 14.III ; $n^{\circ} 7$, Z 14.III.10. Racloirs : $n^{\circ} 5$, W 13.III.18; $n^{\circ} 6$, W 12.III.17 et 8. Pièce bifaciale : $n^{\circ} 4, A$ 14.III.6.

Gonesse (Val-d'Oise), La Fosse aux Larrons: lithic industry from layer 2 . Arrowheads: $n^{\circ} 1, W$ 12.III.6; $n^{\circ} 2, Z$ 17.III.1. Scrapers: $n^{\circ} 3, Z$ 14.III ; $n^{\circ} 7$, Z 14.III.10. Racloirs: $n^{\circ} 5, W$ 13.III.18; $n^{\circ} 6, W 12 . I I I .17$ et 8. Bifacial piece: $n^{\circ} 4$, A 14.III.6.

gauche sont fracturées (W 13.II.1, silex beige à patine blancbleutée, $2,1 \times 1,8 \times 0,35 \mathrm{~cm}$ : fig. 20, nº 4).

Pièce foliacée : c'est un fragment sur support mince à courtes retouches bifaciales bilatérales obliques, pointe et base sont fracturées (Z 15.II.10, silex gris, 2,6 × 2,5 x 0,4 cm : fig. 20, $\mathrm{n}^{\circ} 5$ ).
Grattoirs : deux pièces de morphologie différente, l'une allongée à base fracturée comme une partie du front obtenu par courtes retouches directes ultra-abruptes (X 15.II, silex beige avec patine incipiente blanchâtre, $5,1 \times 3,1 \times 1 \mathrm{~cm}$ : fig. 20 , $n^{\circ}$ 7), l'autre ovalaire à dos aux deux-tiers cortical avec un front périphérique dont la moitié droite est façonnée par retouches 
semi-abruptes directes et la moitié gauche par courtes retouches obliques directes (Z 13.II.1, silex brun à patine blanc-bleutée, 4,4 x 3,4 x 1,2 cm : fig. 20, $\left.\mathrm{n}^{\circ} 9\right)$.

Racloirs : trois pièces sur éclat dont la première a son tranchant obtenu par courtes retouches rasantes sur le bord gauche fracturé à son extrémité (A 14.II, silex beige, 3,5 x $3,1 \times 0,9 \mathrm{~cm}$ : fig. $20, \mathrm{n}^{\circ} 10$ ), la seconde un front transversal oblique rectiligne par courtes retouches inverses obliques sur lesquelles s'est développée une patine blanche couvrante (Y 14.II, silex beige à patine blanche, $4,6 \times 4,3 \times 1 \mathrm{~cm}$ : fig. $20, \mathrm{n}^{\circ}$ 12) ; quant à la troisième à dos partiellement cortical, très petite à base fracturée, elle présente une retouche périphérique semiabrupte directe formant partiellement corniche et donnant un front surélevé (A 13.II, silex à patine blanche, 2 x 1,3 x 0,8 cm : fig. 20, n $n^{\circ}$ ).

Burin : il est dièdre d'angle distal sur éclat épais dont la zone du bulbe a été amincie par retouches rasantes et dont le bord gauche opposé aux coups de burin a été repris par retouches semi-abruptes directes (W 15.II, silex brun à patine réticulée blanc-bleutée, 3,9 x 3,4 x 1,6 cm : fig. 20, n 8).

Tranchet : il est réalisé sur un éclat épais utilisé transversalement et façonné par retouches bilatérales partielles : bord gauche plus épais avec vestiges du bulbe aminci par retouches ventrales semi-abruptes accompagnées de retouches directes insistantes pour amincir aussi la face dorsale ; bord droit repris par retouches rasantes inverses et semi-abruptes directes; tranchant transversal vif esquillé (X 13.II.2, silex brun à patine réticulée blanc-bleutée, 5,7 x 3,7 x 1,8 cm : fig. 20, $\mathrm{n}^{\circ} 13$ ).

Pièce polie : fragment médian à section ovalaire d'une pièce indéterminée (Y 15.II.6, silex -brun ? - craquelé par le feu, $3,2 \times 2,6 \times 1,8 \mathrm{~cm}$ : fig. $\left.20, \mathrm{n}^{\circ} 11\right)$.

\section{Couche 2 (marquée III), 8 pièces}

Armatures : l'une est triangulaire à tranchant transversal réalisée sur un petit éclat dont le talon en constitue le bord gauche, et l'extrémité le bord droit, partiellement repris par une petite retouche directe abrupte (W 12.III.6, silex à patine blanc-bleutée, $2,1 \times 2,1 \times 0,5 \mathrm{~cm}$ : fig. $21, \mathrm{n}^{\circ} 1$ ), l'autre, façonnée par retouches plates bifaciales envahissantes, est perçante à ailerons pointus dont un brisé comme le pédoncule et conserve une petite plage corticale (Z 17.III.1, silex à patine blanche, $2,4 \times 1,9 \times 0,4 \mathrm{~cm}$ : fig. $\left.21, \mathrm{n}^{\circ} 2\right)$.

Grattoirs : tous deux à partie proximale fracturée et à front mince obtenu par retouches obliques directes mais dans un cas sur un support épais à dos cortical aminci par larges retouches rasantes sur la face ventrale (Z 14.III, silex brun à patine blanc-bleutée, 4,2 x 4,1 x 1,8 cm : fig. 21, n 3), dans l'autre sur un fragment de plaquette thermique mince ( $\mathrm{Z}$ 14.III.10, silex beige, $2 \times 3,8 \times 0,5 \mathrm{~cm}$ : fig. $21, \mathrm{n}^{\circ} 7$ ).

Racloirs : ce sont d'une part un outil bilatéral sur éclat aux bords obliques façonnés par retouches alternes obliques (W 13.III.18, silex brun à patine blanc-bleutée, $4,5 \times 5 \times 1,2 \mathrm{~cm}$ : fig. $21, n^{\circ} 5$ ) et d'autre part une grande plaquette thermique mince partiellement corticale en deux fragments dont deux bords adjacents ont été repris par une courte retouche directe oblique à semi-abrupte créant un double front denticulé légèrement concave (W 12.III.17 et 8 , silex beige à patine blancbleutée, 6,6 x 8,2 x 1,2 cm : fig. 21, $\mathrm{n}^{\circ} 6$ ).

Pièce bifaciale : c'est un objet ovoïde épais d'allure nucléiforme à larges retouches obliques couvrantes bifaciales formant des indentations périphériques, évoquant une ébauche de petite pièce biface (A 14.III.6, silex beige à patine blancbleutée, 6,6 x 5,3 x 2,7 cm : fig. 21, $\mathrm{n}^{\circ} 4$ ).

Pièce polie : un petit éclat indique la présence d'une pièce polie (V 14.III, silex beige brûlé, 1,6 x 1,5 x 0,15 cm).

(Jacques Tarrête)

\section{LA FAUNE}

Les vestiges osseux recueillis lors des fouilles du site de «La Fosse aux Larrons » en 1976 forment un lot d'un peu plus de 2000 pièces dont 328 ont pu faire l'objet d'une attribution spécifique. L'état de conservation de ces pièces est assez variable avec une proportion importante de pièces qui présentent une surface érodée ainsi qu'une incidence non négligeable de l'action de carnivores (près de $3 \%$ des pièces déterminées rongées) ou encore d'exposition au feu (environ $1 \%$ des pièces brûlées).

Leur distribution selon les différentes unités stratigraphiques distinguées lors de la fouille est assez inégale (tabl. XXVII et XXVIII). En effet, un peu plus de la moitié de ces vestiges sont associés à la couche 2, qui correspond à l'horizon sur lequel a été édifié le tumulus, interprété comme un niveau d'habitat, alors que les éléments provenant des couches 4 et 3 et de la chape forment des lots aux effectifs plus modestes (entre 16 et 108 pièces). Ces restes sont caractérisés par un poids moyen de $3,5 \mathrm{~g}$ qui renvoie à un état de fragmentation assez intense : ceci mérite cependant d'être relativisé par la prise en compte des contextes de découverte qui semblent déterminer de manière significative l'état de conservation. Les valeurs de poids moyen varient en effet du simple au double entre les ensembles des couches 4, 3 et la chape et celui de la couche 2 qui concentre les restes les plus nombreux mais aussi les plus conséquents : les effets de la conservation différentielle y apparaissent moins marqués et ils se distinguent par ailleurs par un taux de détermination presque deux fois plus important. Ces variations laissent augurer de caractéristiques différentes qui s'expriment aussi au niveau de la composition de ces deux ensembles : c'est la couche 2 , clairement représentative de la première occupation du site, qui servira de base à cette étude. Toutefois, la prédominance des restes d'animaux domestiques et la discrétion des restes de faune sauvage apparaissent assez constantes d'un lot à l'autre. Avec une contribution moyenne d'environ $15 \%$ du nombre de restes et de près de $19 \%$ du poids (à l'exclusion des fragments de ramure de cerf non inclus dans les décomptes), la contribution de la chasse ne peut cependant être considérée comme tout à fait marginale. Le cortège des gibiers apparaît clairement dominé par le chevreuil auquel se rapportent près de la moitié des pièces, alors que le cerf y apparaît beaucoup plus faiblement représenté. Moins prisé que le chevreuil, le sanglier représente cependant le gibier le plus fréquent après le cerf. Quoique plus discrètes, les espèces comme le blaireau, le chat sauvage et le renard attestent d'une forme de prédation peut-être principalement motivée par la fourrure. Parmi les restes de grande faune figure aussi une molaire inférieure de cheval qui a été attribuée à la forme sauvage.

Les restes d'animaux domestiques se rapportent aux différentes espèces attestées à la fin du Néolithique y compris au chien représenté par une mandibule complète d'un individu assez âgé (stade d'usure D d'après Herbin-Horard, 1997) provenant de la couche 3 et une molaire supérieure isolée parmi 

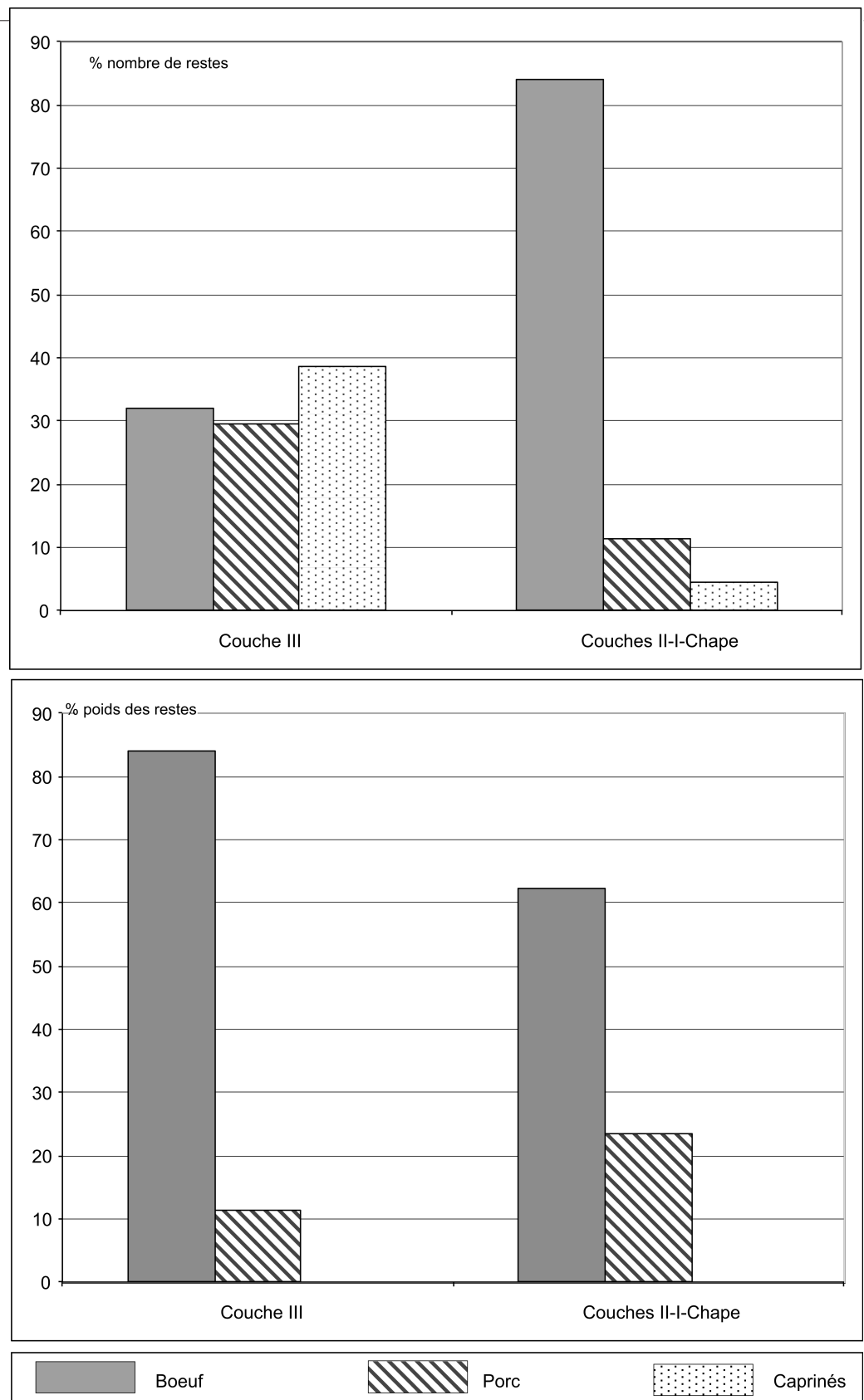

Fig. 22 - Gonesse (Val-d'Oise), La Fosse aux Larrons : importance relative des principales espèces, en nombre et en poids des restes.

Gonesse (Val-d'Oise), La Fosse aux Larrons: relative frequency of the main species, in numbers and weight of remains.

les restes de la couche 4. Cette discrétion peut être liée au statut de cette espèce qui ne peut être confondu avec celui des autres animaux élevés en vue de leur rapport économique, qu'il s'agisse de viande ou d'autres productions (lait, laine, force de travail....). Le lot de la couche 2, qui est le plus conséquent, est caractérisé par une composition assez équilibrée des principales espèces domestiques, avec un léger avantage des caprinés par rapport au bœuf et au porc dont les restes sont un peu moins nombreux (fig. 22). Cette répartition ne se retrouve pas dans les lots provenant des couches 3 et 4 ou de la chape, dont les effectifs sont plus faibles et dont la représentativité semble moins assurée mais qui sont marqués par une contribution plus élevée du porc. Évaluée d'après le poids des restes, l'importance relative des animaux domestiques de la couche 2 apparaît plus classiquement dominée par les restes de bovins qui, en comparaison avec la masse des restes de porc et petits ruminants, est beaucoup plus modeste. Dans le lot provenant de la couche 4 , la place plus importante du porc est bien enregistrée par une contribution en poids des restes supérieure à celle qui caractérise la couche 2 .

Les lots de faune de ces différents ensembles stratigraphiques présentent aussi des compositions anatomiques assez variables (fig. 23 et tabl. XXIX). La caractéristique la plus constante semble résider dans la sur-représentation des dents isolées qui s'observe pour tous les lots, quelle que soit l'espèce considérée y compris pour les animaux chassés en dépit des effectifs 

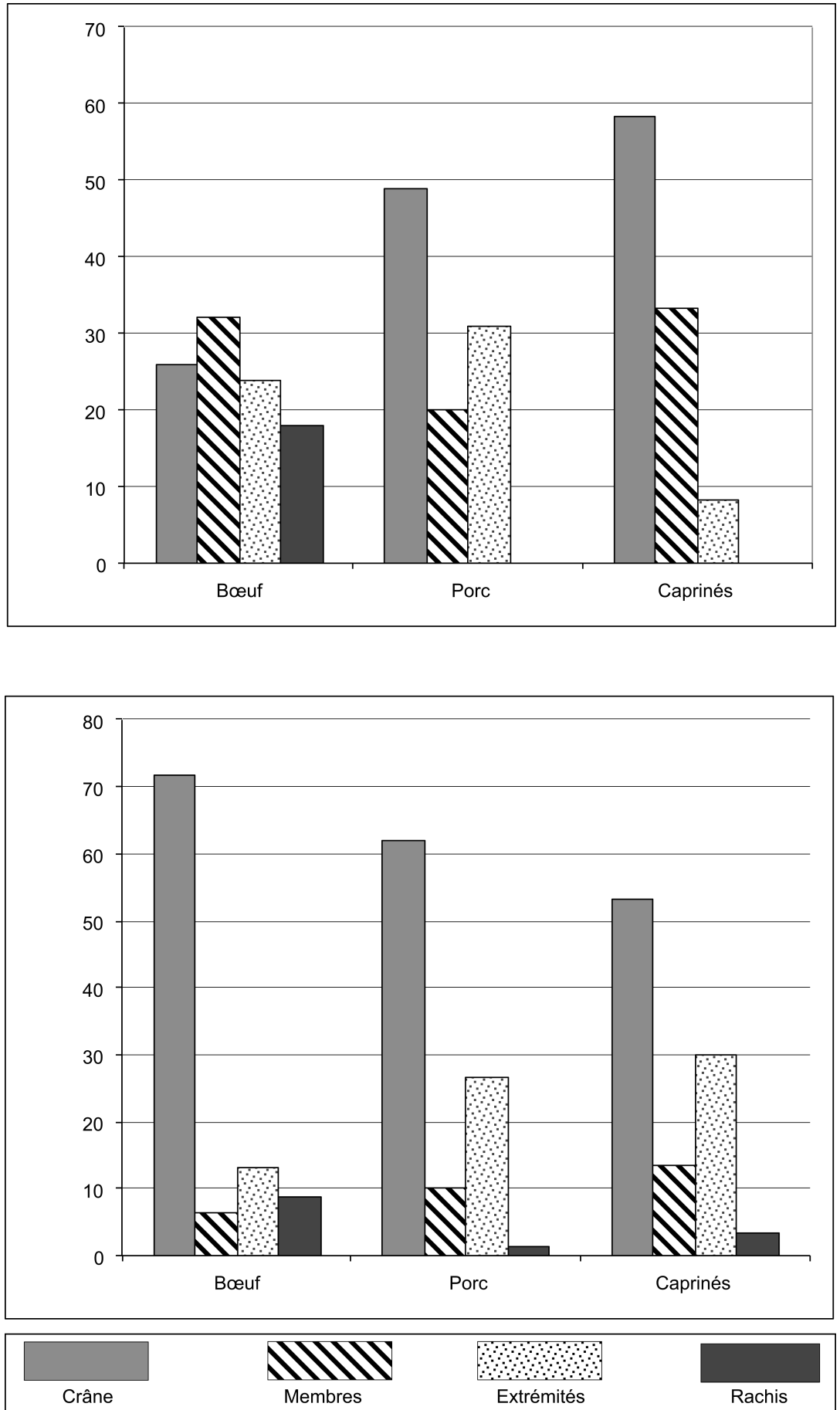

Fig. 23 - Gonesse (Val-d'Oise), La Fosse aux Larrons : répartition des restes des différentes régions anatomiques par ensemble stratigraphique, couche 2 en haut, couches 3, 4 et chape en bas (en \% sur le nombre de restes).

Gonesse (Val-d'Oise), La Fosse aux Larrons: distribution of remains of the various anatomical zones by stratigraphic unit, layer 2 at top, layers 3, 4 and chape below (in \% of the number of remains).

de restes assez modestes. A l'exception du bœuf domestique, pour lequel des restes de toutes les parties de squelettes sont attestées, les répartitions anatomiques apparaissent largement tronquées du fait de l'absence ou de la sous-représentation du rachis (côtes et vertèbres). Cette lacune peut en partie s'expliquer par l'incidence de facteurs taphonomiques à laquelle renvoie aussi l'importance des dents isolées et à laquelle peut être rapportée une conservation différentielle particulièrement prononcée. La représentation systématique des parties des membres et des extrémités s'accorde avec une exploitation bouchère des principaux animaux du cheptel et une valorisation de l'ensemble des carcasses, y compris des bas morceaux. La présence de restes d'extrémités est bien marquée aussi dans le cas du chevreuil et atteste du transport de quartiers complets 


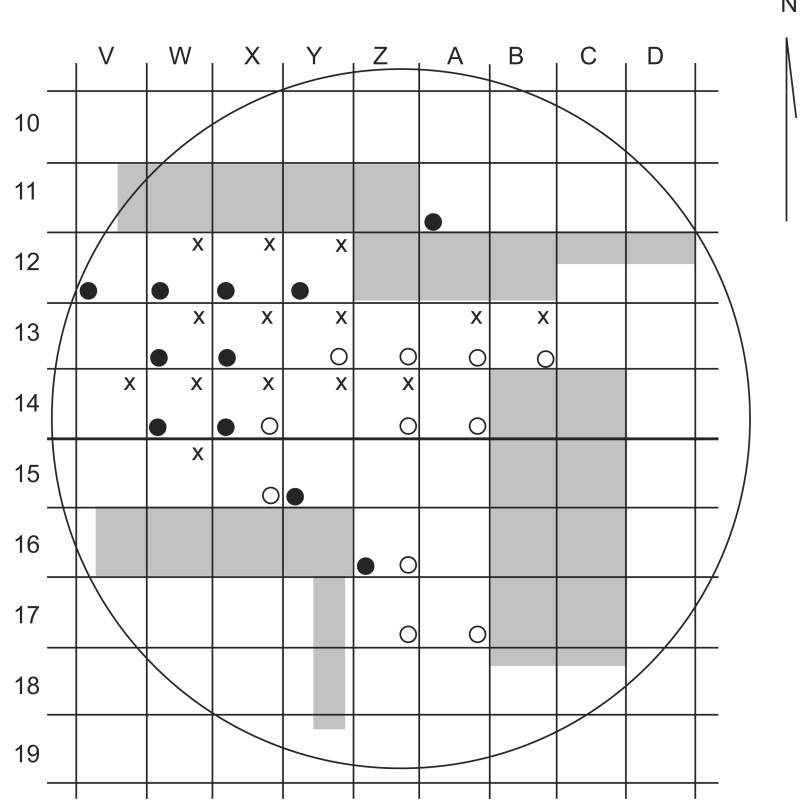

Fig. 24 - Gonesse (Val-d'Oise), La Fosse aux Larrons : plan schématique de concentration des tessons de céramique dans les couches 4 à $2(\geq 5 / \mathrm{m} 2)$ : rond $=4$, point $=3$, croix $=2$; grisé = zones non fouillées.

Gonesse (Val-d'Oise), La Fosse aux Larrons: schematic plan of the concentration of sherds in layers 4 to $2(\geq 5 / \mathrm{m} 2)$ : circle $=4$, point $=3$, cross $=2$; shading $=$ unexcavated zones.

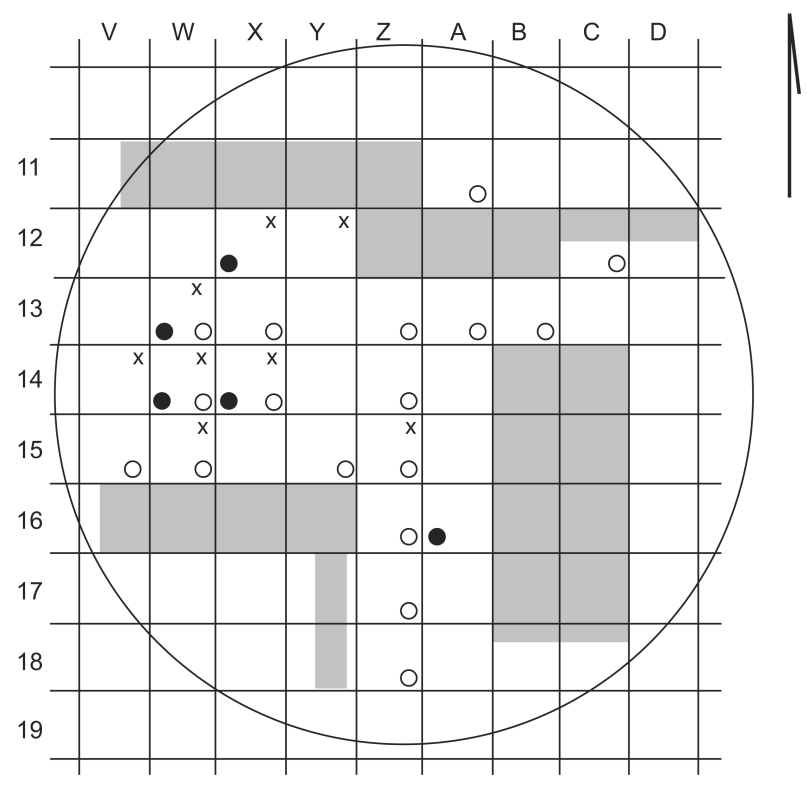

Fig. 25 - Gonesse (Val-d'Oise), La Fosse aux Larrons : plan schématique de concentration des produits lithiques dans les couches 4 à $2(\geq 20 / \mathrm{m} 2)$ : rond $=4$, point $=3$, roix $=2$; grisé = zones non fouillées

Gonesse (Val-d'Oise), La Fosse aux Larrons: schematic plan of the concentration of lithic artefacts in layers 4 to 2 ( $\geq 20 / \mathrm{m} 2)$ : circle $=4$, point $=3$, cross $=2$; shading $=$ unexcavated zones. sur le site, voir de squelettes complets comme semble l'indiquer l'importance des restes dentaires.

Les observations concernant l'âge et le sexe des animaux sont très peu nombreuses et ne permettent pas une approche statistique des règles de sélection des animaux. Les données relatives à l'état d'épiphysation des os du squelette appendiculaire reflètent, dans le cas des bovins, la présence d'une majorité d'animaux adultes tandis que, d'après les données sur les stades d'éruption et d'usure dentaire, la sélection intègre surtout des animaux jeunes parmi lesquels peuvent être identifiés 5 individus de moins de 3 ans ( $\mathrm{m} 4$ usées) et 1 individu de plus de 3 ans (M3 usée). Les restes de porcs domestiques et des petits ruminants se rapportent principalement à des jeunes, voire de très jeunes avec 4 individus de moins d' 1 an ( $\mathrm{m} 4$ en début d'usure ou usée), 1 individu d'environ 1an 1/ 2 (M3 sortie non usée) et 1 individu adulte (M3 très usée) dont 2 mâles et 1 femelle.

Ces indications sont en bonne cohérence avec une exploitation des animaux de type boucher mais n'excluent pas la valorisation d'autres productions comme le lait dont la mise en évidence demanderait alors à être appuyée sur une base documentaire plus large.

L'analyse de ce petit lot de faune laisse entrevoir quelquesunes des caractéristiques de l'exploitation des ressources animales au Néolithique final. Malgré leur mauvais état de conservation général, ces restes peuvent d'après leur état de fragmentation, leur distribution anatomique et les données sur l'âge d'abattage être apparentés à des reliefs de transformation et de consommation même si d'autres formes d'exploitation, pour l'artisanat ou des productions secondaires, ne peuvent être mises en évidence, en raison principalement du faible nombre de restes en présence. La place importante du Bouf, et celle du porc comme principale ressource secondaire, restitue une contribution des principales ressources domestiques qui paraît assez proche de celle mise en évidence sur d'autres sites du même horizon chronologique comme celui de Meaux (77) « Route de Varreddes » (Bémilli, 2007), Houplin-Ancoisne (59) «Le Marais de Santes »(Braguier, 2007) ou encore Valenciennes (59) « Rue Jean Bernier» (Oueslati, 2009).

(R.-M. Arbogast)

\section{RÉPARTITION DES VESTIGES}

On a vu que sur les 568 tessons recueillis, 53 provenaient des couches 5/6, 157 de la 4, 131 de la 3 et 227 de la 2 (tabl. XXVI). $\mathrm{Si}$ on établit leur répartition par $\mathrm{m}^{2}$ dans les couches 2 à 4 et que, pour apprécier leur concentration, on retient au moins 5 tessons par $\mathrm{m}^{2}$, on observe sur un plan schématique (fig. 24) qu'en 4 , ils occupent davantage le secteur sud-est de la partie fouillée alors qu'en 3 et en 2, ils se regroupent vers le nord-ouest avec de fortes concentrations surtout pour cette dernière (jusqu'à plus de 20 pièces) dans les mètres $\mathrm{W}, \mathrm{X}, \mathrm{Y} 12$ à 14.

Le faible nombre des produits lithiques façonnés, réparti entre les 4 couches individualisées, ne permet évidemment pas de les caractériser : observons tout au plus que grattoirs et racloirs s'y retrouvent dans chaque niveau, de même que les armatures tranchantes et que les fragments de pièces polies et 


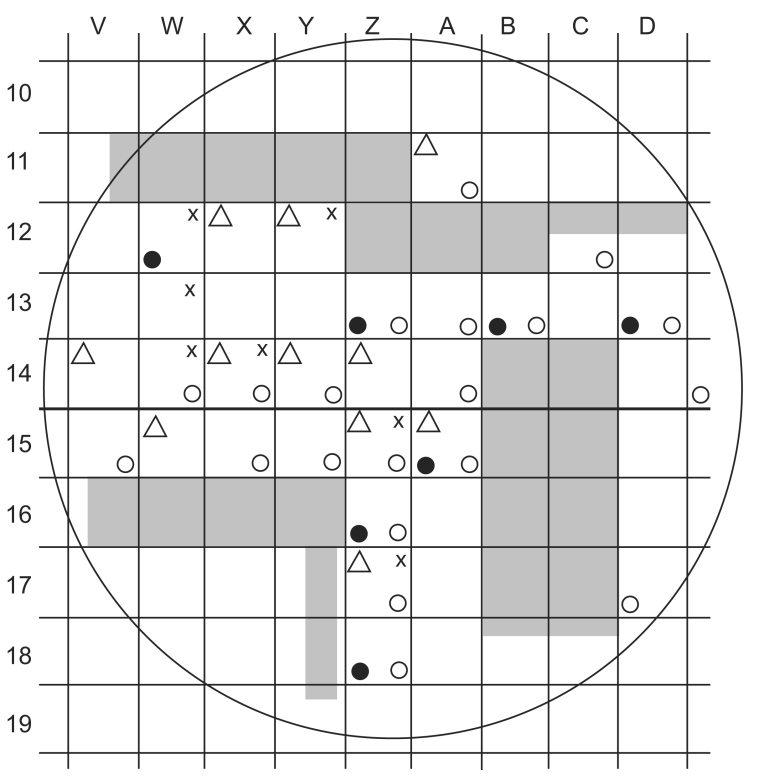

Fig. 26 - Gonesse (Val-d'Oise), La Fosse aux Larrons : plan schématique de concentration des vestiges de faune déterminés des 3 principales espèces $(\geq 5 / \mathrm{m} 2)$ et de l'ensemble des ossements indéterminés $(\geq 10 \mathrm{~m} 2)$ dans les couches 4 (point, rond) et 2 (croix, triangle) ; grisé = zones non fouillées.

Gonesse (Val-d'Oise), La Fosse aux Larrons: schematic plan of the concentration of faunal remains identified to the 3 main species $(\geq 5 / \mathrm{m} 2)$ and of all unidentified bones $(\geq 10 \mathrm{~m} 2)$ in layers 4 (point, circle) and 2 (cross, triangle); hatching = unexcavated zones.

les armatures perçantes existent dans l'ensemble des couches 2 à 4 (cf. tabl. XXX).

Le décompte des produits lithiques par couche montre une certaine concentration, de l'ordre d'une vingtaine de produits ou davantage (cf. plan schématique de répartition, fig. 25) :

pour la chape, dans les carrés $C 9$ à 11 ;

pour la couche 4, dans les carrés V 15, W 13 à 15, X 13-14, Y 15, Z 13 à 18, A 11 et 13, B 13, C 12 ;

pour la couche 3 , dans les carrés W 13-14, X 12 et 14, A 16 ; pour la couche 2, dans les carrés V 14, W 13 à $15, \mathrm{X} 12$ et $14, \mathrm{Y} 12, \mathrm{Z} 15$.

Dans ce dernier cas, la densité des vestiges mais aussi la présence de nombreux blocs de calcaire brûlé faisant songer à la possible existence d'un foyer construit démantelé a conduit à un enregistrement planimétrique des principaux éléments dans les mètres V-W/12 à 15, X 12-13, Y 12 (fig. 14), poursuivi vers l'est de manière schématique, les artéfacts se raréfiant considérablement (fig. 15). Par ailleurs, on relève ainsi 4 secteurs où cette densité concerne l'ensemble des couches 2 à 4 : W 13 et 14, X 14, Z 15. De ce fait, si pour la 2 la concentration principale se retrouve dans le secteur nord-ouest comme pour la céramique, la répartition pour la 3 est beaucoup plus large et pour la 4 elle diffère notablement, située essentiellement en-deçà de la bande des Y.

Pour ce qui est des produits brûlés, si la couche 4 compte à elle seule $40 \%$ de l'ensemble, il apparait que leur nombre reste proportionnel à celui de la totalité des pièces dans chacune des couches 2 à 4 , de l'ordre de $1 / 5^{\text {̀me }}$ et plus fort au niveau de la chape, près d'un tiers. Cette importance dans la couche 4 est sans doute à mettre en relation avec la présence de paquets de sédiment noirâtre qui y a été observée.

Si l'on examine la répartition des vestiges de faune (cf. plan schématique, fig. 26) en sélectionnant, par exemple, les carrés où le nombre d'éléments déterminés est égal ou supérieur à 5 (la couche 3 n'a pas été retenue en raison du trop faible nombre de restes), on observe une concentration dans la partie centrale de la moitié ouest pour la couche 2 (W 12, 13, 14 ; X 14 ; Y 12 ; Z 15 et 17$)$ alors que c'est le secteur sud-est, sauf une exception (W 12), qui concentre la faune de la couche 4 (Z 13, 16, 18 ; A 15 ; B 13 ; D 13). Si l'on ajoute les ossements indéterminés d'un nombre égal ou supérieur à $10 / \mathrm{m}^{2}$, la configuration s'élargit bien sûr pour la couche 2 (s'ajoutent V 14, W 15, X 12, Y 14, $\mathrm{Z}$ 14, A 15) mais demeure dans la même zone à une exception près (A 11) alors qu'elle s'étend largement pour la couche 4 , et notablement vers le centre-ouest (V 15, W 14, X 14 et 15, Y 14 et 15), retrouvant une image proche de celle donnée par la répartition des produits lithiques.

\section{INTERPRÉTATION ET CONCLUSION}

Le site de La Fosse aux Larrons a donc livré une inhumation sous tumulus rapportable à la fin de l'âge du Bronze ancien ou au début de l'âge du Bronze moyen si l'on en croit la datation ${ }^{14} \mathrm{C}$ dont on a vu que la plus grande probabilité la situe entre 1746 et 1208 av. J.-C. (Voruz, 1996) : en l'absence de tout élément mobilier d'accompagnement caractéristique, le seul objet qui pourrait lui être attribué, sans certitude, une pointe en silex sommairement façonnée, est atypique. Rappelons que cette date, obtenue à partir des restes de côtes et vertèbres, concorde remarquablement avec celle issue des éléments organiques carbonisés provenant de la mince couche 7 scellant la chape du tumulus. L'analyse anthropologique détaillée montre clairement qu'il s'agit d'un individu adulte de sexe masculin d'aspect robuste ; l'examen de la disposition des ossements permet de supposer l'existence d'un contenant assez large sans fond rigide et l'analyse palynologique suggère l'hypothèse d'une sorte d'oreiller en lierre, compte-tenu de l'abondance anormale des pollens de cette plante inclus dans le sédiment prélevé sous le crâne. Si on ne peut exclure la présence d'autres sépultures dans la mesure où la fouille du tumulus n'a pu être exhaustive, la situation centrale de l'inhumé accrédite la vraisemblance de son unicité. La structure et la mise en place de cette sépulture posaient toutefois plusieurs questions dans la mesure où il apparaissait une succession de couches diverses contenant notamment à plusieurs niveaux des éléments de mobilier témoignant d'une ou plusieurs occupations humaines. Aussi, dans la description qui précède, a-t-il été choisi de présenter chaque catégorie de vestiges, couche par couche, afin de mettre en évidence leur éventuelle singularité ou au contraire une certaine homogénéité. Pour ce qui est de la céramique, la faible quantité des tessons, leur très médiocre conservation et leur très grande disparité à l'intérieur d'une même couche, n'apportent guère, en dehors de ces constantes, d'indication particulière. Les produits lithiques en revanche, d'une couche à l'autre, présentent une composition analogue dans leur nature, où l'on relève entre autres, l'abondance des esquilles et des cassons, fréquemment brûlés ; en outre, certaines pièces façonnées (armatures, grattoirs, racloirs) sont présentes dans chaque couche. Quant 


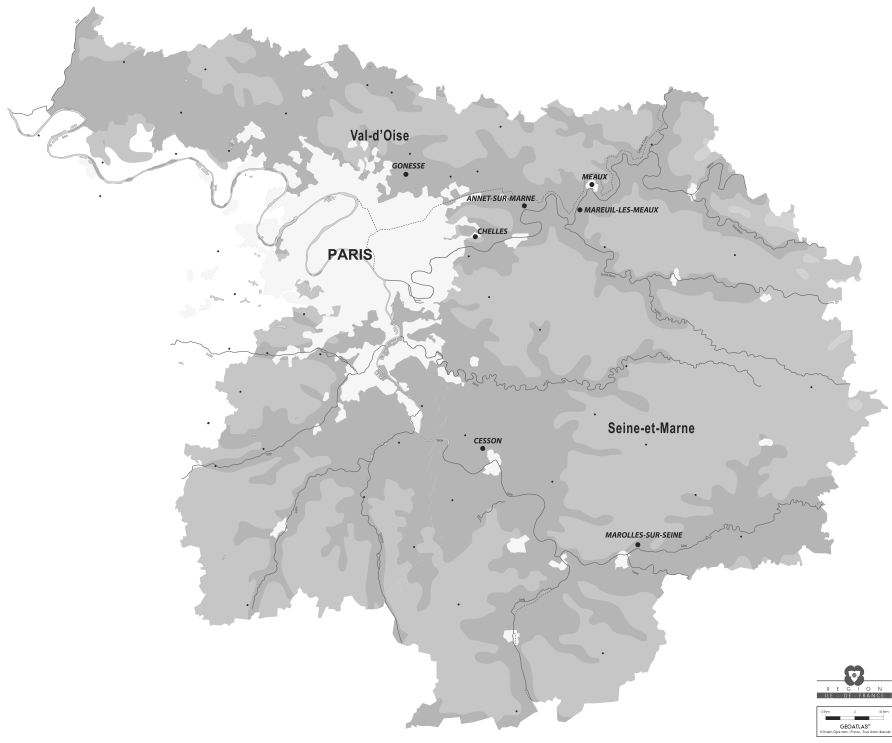

Fig. 27 - Quelques sites de comparaison, du nord au sud: Chelles, Fossé de Travers Ouest ; Annet-sur-Marne, Les Plantards ; Meaux, Route de Varreddes; Mareuil-les-Meaux, Les Vignolles; Cesson, Plaine du Moulin à Vent; Marolles-sur-Seine, La Croix de la Mission (extrait de la carte Geoatlas, région Île-de-France).

Some comparable sites, from north to south: Chelles, Fossé de Travers Ouest; Annet-sur-Marne, Les Plantards; Meaux, Route de Varreddes; Mareuil-les-Meaux, Les Vignolles; Cesson, Plaine du Moulin à Vent; Marolles-sur-Seine, La Croix de la Mission (from the Geoatlas map, île-de-France region).

à la faune, des ossements des trois principales espèces domestiques déterminées sont présents à tous niveaux. L'analyse malacologique, de son côté, montre la grande similarité des couches 3 et 2 , où l'analyse palynologique révèle de son côté une continuité marquée, notamment par la présence de céréales qui disparaissent dans les couches supérieures : l'interprétation géoarchéologique propose d'ailleurs que la couche 3 constitue l'horizon illuvial de la 2.

Si donc des constantes se font jour d'une couche à l'autre dans la nature des vestiges, il existe toutefois des disparités dans leur intégrité entre les couches 4 et 3 et la couche 2 . Les premières en effet présentent des stigmates nets et nombreux de remaniement : plus grande fragmentation des tessons aux bords souvent roulés, fréquentes fractures plus ou moins importantes des produits du silex recoupant la patine, grande fragmentation des ossements animaux entraînant une faible détermination (328 sur 2131 soit environ $15 \%$ ), surface érodée, abondance des dents isolées, faible représentation des côtes et des vertèbres... Dans la couche 2 en revanche, on constate la présence de tessons souvent plus volumineux et au moins deux remontages ainsi que des attributions probables à un même récipient (cf. fig. 14) ; il existe également un racloir en deux fragments remontant à 0,60 $\mathrm{m}$ de distance (W 12.8 et 17, cf. fig. 21, $\mathrm{n}^{\circ}$ 6). Quant à la faune, on y dénombre près de la moitié des restes, les plus conséquents et les moins fragmentés (double de poids en moyenne) et aussi les mieux identifiables. Cette couche en partie préservée, principalement, comme on l'a vu, dans le secteur nord-ouest au sommet du tuf à surface irrégulière, présente bien les caractères d'une couche d'occupation demeurée in situ.
Le noyau du tumulus a été constitué en collectant alentours le sédiment limoneux surmontant le tuf en place : ce sédiment présente des accumulations plus ou moins sombres en paquets de texture variable, plus ou moins riches en débris organiques et en menus vestiges mobiliers divers (tessons de céramique, produits lithiques, ossements animaux). La dimension de ces paquets, de la taille d'un demi-seau à un seau, avait suggéré à M. Brézillon qu'ils pourraient correspondre au contenu de «paniers » prélevés par les constructeurs du tumulus à des points différents du sédiment anthropisé sous-jacent. Quant à la mince couche noirâtre 7 recouvrant l'épaisse chape de terre 6, il l'interprétait comme un apport d'éléments végétaux (mottes de gazon ou autre) qui se seraient décomposés sur place par suite d'enfouissement dans un secteur devenu inondable, hypothèse sur laquelle s'accordent les analyses environnementales.

Quelques sites régionaux apportent des éléments de comparaison partiels pour cette sépulture sous tumulus (fig. 27). On trouve ainsi dans le secteur de La Bassée en Seine-etMarne plusieurs nécropoles où l'on relève plus d'une demidouzaine de sépultures de datation similaire sur ossements inhumés ou incinérés (cf. tabl. XXXI communiqué par Patrick Gouge ; Gouge, Peake, 2005) : si certaines ont été trouvées en association avec des enclos circulaires, parfois au centre, aucune ne présente une couronne de pierres. Grâce à une série de mesures d'âge ${ }^{14} \mathrm{C}$, l'étude chronologique plus détaillée du site de La Croix de la Mission à Marolles-sur-Seine (Peake, Delattre, 2005) a bien montré que les inhumations sans mobilier relevaient du Bronze moyen ; la disposition et l'orientation des corps y est variable. Au nord du département, les trois datations obtenues sur l'ensemble funéraire des Vignolles à Mareuil-lès-Meaux sont également proches, notamment celle de l'inhumation en fosse, un adulte dont la disposition du corps diffère nettement (Cottiaux, Lawrence-Dubovac, 2008). Ce sont des nécropoles à incinérations plus tardives qui livrent des entourages circulaires de pierres calcaires mais de dimensions nettement plus réduites comme à Cesson (Legriel et al., 2010) ou plus au sud, dans le Loiret, à Courcelles (Froquet-Uzel, 2008). En termes d'appartenance culturelle, les spécialistes de la période rattachent ce type d'ensemble à l'entité nordoccidentale ou culture de la Manche (cf. Gouge, Peake, 2005) ou complexe Manche - Mer du Nord (Marcigny, Talon, 2009).

Loccupation préhistorique, sous-jacente à cette structure funéraire encore partiellement en place mais largement remaniée pour la constitution même du tumulus, comporte divers vestiges en association : tessons de céramique, produits de débitage du silex dont quelques dizaines retouchés, ossements animaux fragmentés, blocs de pierre. Le dépôt de cet assemblage dont on a vu qu'il comportait relativement peu d'éléments discriminants pourrait n'avoir précédé que de peu de temps l'édification $\mathrm{du}$ monument et donc se rapporter également à une phase de l'Âge du Bronze légèrement antérieure : on ne peut donc écarter cette hypothèse de prime abord. En effet, contrairement aux productions céramiques et métalliques de cette période, on connaît encore peu ce qui a trait au débitage et à l'outillage lithique (cf. par exemple, Martial, 1995 ; Billard et al., 1994) que le nouvel examen du mobilier de certains sites célèbres fouillés anciennement n'a pas permis d'éclairer (Villes, 1987). Quelques études détaillées ont toutefois été réalisées plus récemment comme celles portant sur le vaste site protohistorique du quartier de Vaise à Lyon (Jacquet, 1998) ou sur l'île 
de Tatihou (Marcigny, Ghesquière, 2003) : on remarque que les armatures y sont rares mais qu'en revanche la caractéristique principale est l'abondance des éclats à retouches marginales qu'on ne retrouve pas à Gonesse. Mais la datation ${ }^{14} \mathrm{C}$ effectuée sur ossement animal de la couche 2, tout comme la composition faunistique, invite plus nettement à chercher des comparaisons dans une période antérieure, au début du Néolithique final. Ainsi, par exemple, une fouille effectuée ces dernières années à Meaux, déjà mentionnée à propos de l'étude de la faune (Bémilli, 2007), offre un important ensemble lithique (Durand, 2007) fait surtout d'éclats larges et épais - même si lames et lamelles existent -, où dominent les éclats retouchés (plus du quart de l'outillage), puis les racloirs (dont le $1 / 3$ à encoches), ensuite denticulés et grattoirs pour environ $10 \%$ chaque, les armatures tranchantes et perçantes dépassant à peine la dizaine. Si le type de support paraît de même nature qu'à Gonesse et si l'on constate également l'absence de microdenticulés, la composition générale diffère notablement. Il faut préciser qu'étant donné le fort remaniement des vestiges mobiliers allant parfois jusqu'au bris partiel, il n'a pas paru possible de retenir parmi les pièces, retouchées volontairement, certains produits présentant des retouches marginales sur une partie de leur bord, au nombre de toutes manières relativement restreint. L'intérêt de la comparaison avec le site meldois est renforcé par la présence de 3 sépultures à inhumation sans mobilier dont une (2411) datée du Bronze ancien (Pariat, 2007) : il s'agit d'un adulte déposé sur le dos aux membres inférieurs repliés sur le côté droit, au membre supérieur gauche ramené sur l'abdomen, le droit replié main reposant sur l'épaule ce qui n'est pas sans ressemblance avec notre « larron », l'existence d'un empierrement résiduel venant compléter le tout. Toujours dans le nord de la Seine-et-Marne, au lieu-dit Les Plantards à Annet-sur-Marne (Tarrête, 1985), ont pu être collectés, à l'occasion d'un décapage industriel, plusieurs milliers de vestiges lithiques comprenant notamment, outre des éclats à retouches partielles, des grattoirs sur éclat court, des denticulés, racloirs, armatures de types variés ...dans une couche à la base de laquelle subsistaient quelques fonds de fosses : n'était la qualité du silex issu des proches minières du Haut-Château à Jablines, quelques points communs avec l'industrie de Gonesse apparaissent. Dans le même secteur géographique, on peut aussi mentionner le site du Fossé de Travers Ouest à Chelles (Tarrête, 1989) à la stratigraphie complexe mais avec une date ${ }^{14} \mathrm{C}$ très proche et dont l'industrie lithique est de composition voisine de celle de Gonesse, notamment par ses armatures tranchantes et à ailerons et pédoncule. Enfin, il faut relever à Gonesse l'absence de racloirs à encoches, de pièces allongées à retouches plates couvrantes de type «poignard » qui, avec les microdenticulés, sont réputés caractériser la fin du Néolithique alors qu'en revanche les armatures tranchantes y sont encore bien attestées : peut-être s'agit-il ici du tout début de cette phase où émergent les groupes du Gord ou de DeûleEscaut, ce qui serait en accord avec la datation radiocarbone.

Quelque trente-cinq ans après sa découverte, le site de La Fosse aux Larrons à Gonesse, malgré les limites apportées à son étude en raison de conditions de gisement et de fouille difficiles et son exploitation partielle, apporte de nouvelles données sur des périodes où la documentation demeure encore peu abondante et souvent imprécise non seulement en Ile-de-France mais dans tout le nord de la France.

Tabl. XXVI - Gonesse (Val-d'Oise), La Fosse aux Larrons : caractérisation des tessons de céramique par couche.

Gonesse (Val-d'Oise), La Fosse aux Larrons: characterization of pottery sherds by layer.

\begin{tabular}{|l|c|c|c|c|}
\hline Couleur des parois & \multicolumn{1}{c}{ chape } & \multicolumn{1}{c}{ couche $\mathbf{l}$} & \multicolumn{1}{c|}{ couche 3 } & \multicolumn{1}{c|}{ couche 2 } \\
\hline 2 beiges & 19 & 77 & 73 & 143 \\
\hline 2 orangées & 9 & & 8 & \\
\hline 1 beige/1 grise & 14 & 36 & 22 & \\
\hline 2 grises & 9 & 25 & 19 & 34 \\
\hline 2 gris beige & & 7 & 9 & \\
\hline 2 rougeâtres & & 4 & & \\
\hline 2 beige-orangé & & 5 & & 48 \\
\hline divers & 2 & 3 & & 2 \\
\hline Dimensions & & & & \\
\hline 1 à $1,4 \mathrm{~cm}$ & 10 & 21 & 23 & 17 \\
\hline 1,5 à $1,9 \mathrm{~cm}$ & 15 & 50 & 45 & 51 \\
\hline 2 à $2,5 \mathrm{~cm}$ & 22 & 53 & 36 & 61 \\
\hline$>2,5 \mathrm{~cm}$ & 6 & 33 & 27 & 98 \\
\hline Total & $\mathbf{5 3}$ & $\mathbf{1 5 7}$ & $\mathbf{1 3 1}$ & $\mathbf{2 2 7}$ \\
\hline
\end{tabular}




\begin{tabular}{|l|c|c|c|c|c|c|c|}
\hline \multicolumn{7}{|c|}{ Décomptes en nombre de restes } \\
\hline & Couche 2 & $\%$ & Couche 3 & Couche 4 & Chape & Total & $\%$ \\
\hline Bœuf & 38 & 26,57 & 11 & 28 & 7 & 46 & 24,86 \\
\hline Porc & 35 & 24,48 & 23 & 49 & 7 & 79 & 42,70 \\
\hline Caprinés & 46 & 32,17 & 11 & 18 & 1 & 30 & 16,22 \\
\hline Chien & & & 1 & 1 & & 2 & 1,08 \\
\hline Total domestiques & $\mathbf{1 1 9}$ & $\mathbf{8 3 , 2 2}$ & $\mathbf{4 6}$ & $\mathbf{9 6}$ & $\mathbf{1 5}$ & $\mathbf{1 5 7}$ & $\mathbf{8 4 , 8 6}$ \\
\hline Cheval & & & & 1 & & 1 & 0,54 \\
\hline Cerf* & 2 & 1,40 & 1 & 3 & & 4 & 2,16 \\
\hline Chevreuil & 13 & 9,09 & 9 & & & 9 & 4,86 \\
\hline Sanglier & 4 & 2,80 & 2 & 5 & & 7 & 3,78 \\
\hline Blaireau & 5 & 3,50 & 2 & 2 & & 4 & 2,16 \\
\hline Renard & & & & 1 & 1 & 0,54 \\
\hline Chat sauvage & & & 1 & & & 1 & 0,54 \\
\hline Mustélidés & & & & 1 & & 1 & 0,54 \\
\hline Total sauvages & $\mathbf{2 4}$ & $\mathbf{1 6 , 7 8}$ & $\mathbf{1 5}$ & $\mathbf{1 2}$ & $\mathbf{1}$ & $\mathbf{2 8}$ & $\mathbf{1 5 , 1 4}$ \\
\hline Total déterminés & $\mathbf{1 4 3}$ & $\mathbf{1 0 0 , 0 0}$ & $\mathbf{6 1}$ & $\mathbf{1 0 8}$ & $\mathbf{1 6}$ & $\mathbf{1 8 5}$ & $\mathbf{1 0 0 , 0 0}$ \\
\hline Grands ruminants & & & 1 & & & 1 & \\
\hline Petits ruminants & 1 & & 1 & & & 1 & \\
\hline Carnivores & & & 1 & & 1 & 2 & \\
\hline Batraciens & 2 & & & & & 0 & \\
\hline Oiseaux & & & 2 & & & 2 & \\
\hline Micromammifères & & & 1 & 2 & & 3 & \\
\hline Indéterminés & 261 & & 166 & 584 & 124 & 874 & \\
\hline Total & $\mathbf{6 9 3}$ & & $\mathbf{3 5 5}$ & $\mathbf{9 1 0}$ & $\mathbf{1 7 3}$ & $\mathbf{1 4 3 8}$ & \\
\hline & & & & & & & \\
\hline
\end{tabular}

Tabl. XXVII - Gonesse (Val-d'Oise), La Fosse aux Larrons : décomptes des restes de faune (en nombre de restes) par espèce et par ensemble stratigraphique.

Gonesse (Val-d'Oise), La Fosse aux Larrons: counts of faunal remains (numbers of remains) by species and by stratigraphic group.

\begin{tabular}{|c|c|c|c|c|c|c|c|}
\hline \multicolumn{8}{|c|}{ Décomptes en poids des restes (gr.) } \\
\hline & Couche 2 & $\%$ & Couche 3 & Couche 4 & Chape & Total & $\%$ \\
\hline Bœuf & 869 & 72,18 & 76 & 230 & 78 & 384 & 46,18 \\
\hline Porc & 118 & 9,80 & 29 & 107,5 & 8 & 144,5 & 17,38 \\
\hline Caprinés & 46 & 3,82 & 16 & 38 & 1 & 55 & 6,61 \\
\hline Chien & & & 32 & 2 & & 34 & 4,09 \\
\hline Total domestiques & 1033 & $\mathbf{8 5 , 8 0}$ & 153 & 377,5 & 87 & 617,5 & 74,26 \\
\hline \multicolumn{8}{|l|}{ Cheval } \\
\hline Cerf & 100 & 8,31 & 6 & 42 & & 48 & 5,77 \\
\hline Chevreuil & 32 & 2,66 & 28 & & & 28 & 3,37 \\
\hline Sanglier & 34 & 2,82 & 8 & 120 & & 128 & 15,39 \\
\hline Blaireau & 5 & 0,42 & 4 & 2 & & 6 & 0,72 \\
\hline Renard & & & & & 2 & 2 & 0,24 \\
\hline Chat sauvage & & & 2 & & & 2 & 0,24 \\
\hline Mustélidés & & & & & & 0 & 0,00 \\
\hline Total sauvages & 171 & 14,20 & 48 & 164 & 2 & 214 & 25,74 \\
\hline Total déterminés & 1204 & 100,00 & 201 & 541,5 & 89 & 831,5 & 100,00 \\
\hline Grands ruminants & & & 1 & & & & \\
\hline Petits ruminants & 2 & & 2 & & & & \\
\hline \multicolumn{8}{|l|}{ Carnivores } \\
\hline \multicolumn{8}{|l|}{ Batraciens } \\
\hline \multicolumn{8}{|l|}{ Oiseaux } \\
\hline \multicolumn{8}{|l|}{ Micromammifères } \\
\hline Indéterminés & 276 & & 272 & 500 & 126 & 898 & \\
\hline Total & 3890 & & 878 & 2124,5 & 393 & 3395,5 & \\
\hline
\end{tabular}

Tabl. XXVIII - Gonesse (Val-d'Oise), La Fosse aux Larrons : décomptes des restes de faune (en poids de restes) par espèce et par ensemble stratigraphique.

Gonesse (Val-d'Oise), La Fosse aux Larrons: counts of faunal remains (weight of remains) by species and by stratigraphic group. 
Tabl. XXIX - Gonesse (Val-d'Oise),

La Fosse aux Larrons : décomptes des restes des différentes espèces par origine anatomique.

Gonesse (Val-d'Oise), La Fosse aux Larrons : counts of remains of different species by anatomical origin.

\begin{tabular}{|l|l|l|l|l|l|l|l|l|l|l|l|l|l|c|}
\hline \multicolumn{1}{|c|}{ Bœuf } & 2 & 3 & 4 & Chape & \multicolumn{1}{c|}{ Porc } & 2 & 3 & 4 & Chape & \multicolumn{1}{|c|}{ Caprinés } & 2 & 3 & 4 & Chape \\
\hline Crâne & 3 & 1 & 1 & 6 & Crâne & 1 & 1 & 7 & 1 & Crâne & 1 & & & \\
\hline Dent isolée & 7 & 7 & 18 & & Dent isolée & 16 & 11 & 25 & 4 & Dent isolée & 6 & 8 & 7 & 1 \\
\hline Scapula & 3 & & & & Scapula & 4 & 1 & & & Scapula & & & & \\
\hline Humérus & 2 & & & & Humérus & & & 1 & & Humérus & & & 2 & \\
\hline Radius & 1 & & 1 & & Radius & & & & & Radius & 1 & & 1 & \\
\hline Ulna & & & & & Ulna & 2 & & 2 & & Ulna & 1 & & & \\
\hline Carpes & 1 & & & & Carpes & 3 & & 1 & & Carpes & & & & \\
\hline Métacarpe & 1 & & & & Métacarpe & 1 & & 1 & & Métacarpe & & 1 & 1 & \\
\hline Coxal & 2 & & 1 & & Coxal & & & 1 & & Coxal & & & & \\
\hline Fémur & 1 & & & & Fémur & & & & & Fémur & 1 & & & \\
\hline Patella & & & & & Patella & & & & & Patella & & & & \\
\hline Tibia & 3 & & & & Tibia & 1 & & 2 & & Tibia & & & 1 & \\
\hline Tarses & 5 & & & 1 & Fibula & & & & 1 & Tarses & & & 4 & \\
\hline Métatarse & & 1 & & & Tarses & 2 & 2 & 2 & & Métatarse & & 1 & & \\
\hline Phalanges & 2 & & 4 & & Métatarse & 3 & 3 & & & Phalanges & 1 & & 1 & \\
\hline Sésamoïdes & & & & & Phalanges & 2 & 5 & 6 & 1 & Sésamoïdes & & 1 & \\
\hline Vertèbres & 3 & 2 & 2 & & Sésamoïdes & & & & & Vertèbres & & & \\
\hline Côtes & 4 & & & & Vertèbres & & & 1 & & Côtes & & & 1 \\
\hline Os longs & & & 1 & & Côtes & & & & & Os longs & 1 & & & \\
\hline Total & $\mathbf{3 8}$ & $\mathbf{1 1}$ & $\mathbf{2 8}$ & $\mathbf{7}$ & Total & $\mathbf{3 5}$ & $\mathbf{2 3}$ & $\mathbf{4 9}$ & $\mathbf{7}$ & Total & $\mathbf{1 2}$ & $\mathbf{1 1}$ & $\mathbf{1 8}$ & $\mathbf{1}$ \\
\hline
\end{tabular}

\begin{tabular}{|l|l|l|l|l|l|l|l|l|l|l|l|}
\hline Chevreuil & $\mathbf{2}$ & $\mathbf{3}$ & & \multicolumn{1}{|c|}{ Sanglier } & $\mathbf{2}$ & $\mathbf{3}$ & $\mathbf{4}$ & \multicolumn{1}{c}{ Cerf } & $\mathbf{2}$ & $\mathbf{3}$ & $\mathbf{4}$ \\
\hline Crâne & & & & Crâne & & & & Crâne & & 1 & 1 \\
\hline Dent isolée & 3 & 5 & & Dent isolée & 3 & 2 & 5 & Dent isolée & & & \\
\hline Scapula & 1 & & & Scapula & & & & Scapula & & & \\
\hline Humérus & & 1 & & Humérus & & & & Humérus & & & \\
\hline Radius & & & & Radius & & & & Radius & 1 & & \\
\hline Ulna & & & & Ulna & & & & Ulna & & & \\
\hline Carpes & & & & Carpes & & & & Carpes & & & \\
\hline Métacarpe & & & & Métacarpe & & & & Métacarpe & & & \\
\hline Coxal & & & & Coxal & & & & Coxal & & & \\
\hline Fémur & & 1 & & Fémur & & & & Fémur & & & \\
\hline Patella & & & & Patella & & & & Patella & & & \\
\hline Tibia & & & & Tibia & & & & Tibia & & & \\
\hline Tarses & 1 & & & Tarses & 1 & & & Tarses & 1 & & 1 \\
\hline Métapode & 2 & 2 & & Métatarse & & & & Métatarse & & & \\
\hline Phalanges & 6 & & & Phalanges & & & & Phalanges & & & 1 \\
\hline Sésamoïdes & & & & Sésamoïdes & & & & Sésamoïdes & & & \\
\hline Vertèbres & & & & Vertèbres & & & & Vertèbres & & & \\
\hline Côtes & & & & Côtes & & & & Côtes & & & \\
\hline Os longs & & & & Os longs & & & & Os longs & & & \\
\hline Total & $\mathbf{1 3}$ & $\mathbf{9}$ & & Total & $\mathbf{4}$ & $\mathbf{2}$ & $\mathbf{5}$ & Total & $\mathbf{2}$ & $\mathbf{1}$ & $\mathbf{3}$ \\
\hline
\end{tabular}


Tabl. XXX - Décompte schématique de l'outillage lithique par couche.

Schematic count of lithic tools by layer.

\begin{tabular}{|c|c|c|c|c|}
\hline outillage & chape & couche 4 & couche 3 & couche 2 \\
\hline armature tr. transversal & 2 & 2 & 3 & 1 \\
\hline armature à ail. et péd. & & 3 & 1 & 1 \\
\hline armature losangique & & 1 & & \\
\hline pièce foliacée & & & 2 & 2 \\
\hline grattoir & 3 & 2 & 3 & 2 \\
\hline racloir & 1 & 2 & 1 & \\
\hline lame à troncature & & 1 & 1 & \\
\hline tranchet & & & & 1 \\
\hline burin & & 1 & & 1 \\
\hline pointe & & & & \\
\hline pièce biface & & & & \\
\hline pièce polie & & & & \\
\hline
\end{tabular}

Tabl. XXXI - Série de datations ${ }^{14} \mathrm{C}$ effectuées sur des inhumations d'âge comparable à celle de Gonesse provenant de

La Bassée en Seine-et-Marne (document P. Gouge, Conseil général 77).

Series of ${ }^{14} \mathrm{C}$ dates on burials of comparable age to Gonesse from La Bassée in Seine-et-Marne

(document $P$. Gouge, Conseil général 77).

\begin{tabular}{|c|c|cc|c|c|l|}
\hline$N^{\circ}$ labo & Date BP & Date calibrée $(95,4 \%)$ & matériau & $N^{\circ}$ structure & Provenance (commune, lieu-dit) \\
\hline GrA-18031 & $3285 \pm 40$ & $1690 \mathrm{BC}$ & $1410 \mathrm{BC}$ & os humain & St.1 & Marolles-sur-Seine, la Croix-de-la-Mission \\
\hline Ly-9124 & $3265 \pm 42$ & $1680 \mathrm{BC}$ & $1430 \mathrm{BC}$ & os humain & St.291 & Varennes-sur-Seine, le Marais-du-Pont \\
\hline GrA-17945 & $3240 \pm 50$ & $1680 \mathrm{BC}$ & $1400 \mathrm{BC}$ & os humain & St.102 & Varennes-sur-Seine, le Marais-du-Pont \\
\hline GrA-30175 & $3195 \pm 40$ & $1530 \mathrm{BC}$ & $1390 \mathrm{BC}$ & os humain & Inh.15 & Châtenay-sur-Seine, Les Sécherons \\
\hline GrA-17981 & $3170 \pm 50$ & $1530 \mathrm{BC}$ & $1310 \mathrm{BC}$ & os humain & St.38 & Marolles-sur-Seine, la Croix-de-la-Mission \\
\hline Ly-8943 & $3160 \pm 60$ & $1600 \mathrm{BC}$ & $1260 \mathrm{BC}$ & os humain & Sép.5 & Égligny, la Pêcherie \\
\hline GrA-17937 & $3130 \pm 50$ & $1520 \mathrm{BC}$ & $1260 \mathrm{BC}$ & os humain & St.48 & Marolles-sur-Seine, la Croix-de-la-Mission \\
\hline GrA-17985 & $3080 \pm 50$ & $1450 \mathrm{BC}$ & $1210 \mathrm{BC}$ & os humain & St.13 & Marolles-sur-Seine, la Croix-de-la-Mission \\
\hline
\end{tabular}




\title{
BIBLIOGRAPHIE
}

\author{
Abréviations \\ BAFEQ Bulletin de l'Association Française pour l'Étude du Quaternaire \\ BAPRABBulletin de l'Association pour la Promotion des Recherches sur l'Âge du Bronze \\ BCGC Bulletin du Centre de Géomorphologie de Caen \\ BJPGF Bulletin des Jeunesses préhistoriques et géologiques de France \\ BMSAP Bulletins et Mémoires de la Société d'Anthropologie de Paris \\ BSPF Bulletin de la Société préhistorique française \\ CPN Les Cahiers de Préhistoire du Nord \\ CREPA Centre de Recherches et d'Etudes Préhistoriques de l'Auvergne \\ MSPF Mémoires de la Société préhistorique française \\ RAO Revue archéologique de l'Ouest \\ RACF Revue Archéologique du Centre de la France \\ RAE Revue archéologique de l'Est \\ RAIF Revue archéologique d'Île-de-France \\ RAP Revue Archéologique de Picardie \\ RPP Revue de Paléobotanique et de Palynologie \\ SPF Société préhistorique française \\ VHA Vegetation History and Archaeobotany
}

Bibliographie palynologique

Aaby B.

1986: «Trees as anthropogenic indicators in regional pollen diagrams from eastern Denmark » in BEHRE K.-E. (ED.), Anthropogenic Indicators in Pollen Diagrams, Rotterdam, A.A. Balkema, p. 73-93.

\section{BAHAIN J.-J.}

1997 : «Louvres "Le Bois d'Orville” (95 351001 AH) (Val-d'Oise), Compterendu de sondages géologiques » in Chopelain P. et al., Louvres " Le Bois d'Orville» (Val-d'Oise). Fouilles archéologiques d'un habitat du Haut Moyen-Age. Situation géographique et contexte géologique, archéologique et historique, D.F.S., S.R.A. d'Ile-deFrance, Conseil Général du Val-d'Oise, S.D.A.V.O., A.F.A.N., 1998, 25 p.

\section{BARTHÉLÉMY L.}

1976 : Recherches sur les relations entre les pluies polliniques stationnelles et les paysages végétaux avoisinants, Cessières, Aisne, France, Thèse de Doctorat, Université Paris X, 154 p.

\section{BARTHÉLÉMY L.}

1985 : «Réflexions sur la répartition du pollen. Conséquences pour l'archéologie », Palynologie archéologique, actes des journées des 25-26-27 janvier 1984, Sophia Antipolis, CNRS-CRA, Notes et Monographies techniques, 17, p. 53-85.
BEHRE K.-E.,

1981 : «The interpretation of anthropogenic indicators in pollen diagrams », Pollen et Spores, XXIII, 2, p. 225-243.

Bouchet F., Krier V., Leroyer Ch., Monnet C., Pepe Cl., Ruas M. -P., Thiebault S.,

1992: «Fonctions et utilisations. Utilisation attestée » in MONNET C. (Dir.)L'évacuation des déchets en milieu urbain au bas Moyen Age. L'exemple de la Cour Napoléon du Louvre, Louvain, éd. Université catholique, vol. VIII (coll. d'archéologie Joseph Mertens), p. 20-29.

BoUrnerias M.,

1979 : Guide des groupements végétaux de la région parisienne, 3 e éd. Sedes-Masson 1984, $483 \mathrm{p}$.

BREZILLON M.,

1977 : « Informations archéologiques. Circonscription d'Île-de-France. Vald'Oise. Gonesse ", Gallia Préhistoire, CNRS Éditions, Paris, 20, 2, p. 354-355.

BRUN C.,

2007 : Archéophytes et néophytes. Pour une nouvelle détermination des marqueurs polliniques de l'anthropisation. Le cas des milieux cultivés et rudéraux de Franche-Comté, Thèse, Université de Besançon, $421 \mathrm{p}$.

Cour P.,

1974 : « Nouvelles techniques de détection des flux et retombées polliniques ; étude de la sédimentation des pollens et des spores à la surface du sol », Pollen et Spores, XVI, 1, p. 103-141.

Cushing E.J.,

1967 : Evidence for differential pollen preservation in Late-Quaternary sediments in Minnesota, RPP, 4, p. 87-101.

Firmin G.,

1977 : Analyse palynologique de dix échantillons provenant d'un tumulus de l'âge du Bronze situé à Gonesse (Val d'Oise) au lieu-dit «La Fosse aux Larrons ». Rapport d'analyses, 7 p.

Firmin G.,

1984 : « L'empreinte humaine sur les paléomilieux de la vallée de l'Aisne entre Soissons et Cuiry-les-Chaudardes d'après les analyses polliniques de sédiment provenant des fouilles protohistoriques de l'U.R.A. 12 - Université Paris I » in Influences méridionales dans l'Est et le Centre-Est de la France au Néolithique : le rôle du Massif central, Actes du 8e colloque interrégional sur le Néolithique, Le Puy-enVelay, 3-4 octobre 1981, ClermontFerrand, CREPA (cahier $n^{\circ}$ 1), Thème 4 , p. 317-320.

Gaillard M.-J., Sugita S., Bunting .J., Middleton R., Broström A., Caseldine C., Giesecke T., Hellman S.E.V., Hicks S., HJelle ., Langdon C., Nielsen A.-B., Poska A., Von Stedingk H., 
Veski S., Pollandcal members,

2008 : « The use of modelling and simulation approach in reconstructing past landscapes from fossil pollen data : a review and results from the POLLANDCAL network », VHA, 17, 5, p. 419-443.

Girard M. et Renault-Miskovsky J.,

1969 : « Nouvelles techniques de préparation en Palynologie appliquées à 3 sédiments du Quaternaire final de l'abri Cornille (Istres, Bouches- du-Rhône) », BAFEQ, 4, p. 275-284.

Havinga A.J.,

1984 : « A 20-years experimental investigation into the differential corrosion susceptibility of pollen and spores in various soil types », Pollen et Spores, XXVI, 3-4, p. 541-558.

HeIM J.

1970 : Les relations entre les spectres polliniques récents et la végétation actuelle en Europe occidentale, Thèse d'État de l'Université de Louvain (Belgique), 1 vol., $181 \mathrm{p}$.

JALUT G.

1991 : « Le pollen traducteur du paysage agraire », in Guilaine J. (Dir.), Pour une archéologie agraire, Paris, éd. Armand Colin, p. 345-368.

\section{LEBRET $P$.}

1989 : «Tuf du Moulin de la Planche (commune de Gonesse - 95 - vallée du Crould) » in Lecolle F. (Dir.), Les tufs et travertins quaternaires des bassins de la Seine et de la Somme et du littoral cauchois, essai d'inventaire, BCGC, Travaux du groupe Seine 4, novembre, 37 , non paginé.

\section{Leroyer CH.}

1994 : « Les analyses polliniques », in Baray L. et al. (Dir.), D.A.F., 44. Paris, éd. MSH, p. 173-186.

\section{Leroyer Ch.}

1997 : Homme, Climat, Végétation au Tardi-et Postglaciaire dans le Bassin parisien : apports de l'étude palynologique des fonds de vallée, Thèse de l'Université de Paris I, 2 vol., 786 p.

\section{Leroyer Ch.}

2003 : «Environnement végétal des structures funéraires et anthropisation du milieu durant le Néolithique récent/ final dans le Bassin parisien », in Sans dessus dessous - La recherche du sens en préhistoire. Recueil d'études offert à J. Leclerc et $C l$. Masset, RAP, $\mathrm{n}^{\circ}$ spécial 21, p. 83-92.

2006a : «L'environnement végétal des sites : les données de la palynologie », in Malrain F. et Pinard E. (Dir.), Les sites laténiens de la moyenne vallée de l'Oise du Ve au Ier s. avant notre ère. Contribution à l'Histoire de la société gauloise, $R A P, \mathrm{n}^{\circ}$ spécial 23, p. 34-42. 2006b : « L'impact des groupes néolithiques du Bassin parisien sur le milieu végétal : évolution et approche territoriale d'après les données polliniques », in Duhamel P. (Dir.), Impacts interculturels au Néolithique moyen. Du terroir au territoire: sociétés et espaces, Actes $d u 25^{e}$ colloque interrégional sur le Néolithique, Dijon 2001, RAE, 25 ${ }^{\mathrm{e}}$ supplément, p. 133-150.

\section{Leroyer Ch., Allenet G.}

2006 : « L'anthropisation du paysage végétal d'après les données polliniques : l'exemple des fonds de vallées du Bassin parisien », in AlleE Ph., LESPEZ L. (Dir.), L'érosion entre Société, Climat et Paléoenvironnement, Presses Universitaires Blaise Pascal (coll.: Nature et Société n³), p. 63-72

Pastre J.-F., Cecchini M., Dietrich A., Fontugne M., Gauthier A., Kuzucuoglu C., Leroyer Ch., LIMONDIN N.

1991 : «L'évolution holocène des fonds de vallées au nord-est de la Région parisienne (France) : premiers résultats », Physio-Géo, 22-23, p. 109-115.

Pastre J.-F., Limondin-Lozouet N., Gebhardt A., Leroyer Ch., Fontugne M., Krier V.

2001 : « Lateglacial and Holocene fluvial records from the central part of the Paris basin (France) », in MADDY D., Macklin M.G., Woodward J.C. (Eds.), River Basin Sediment Systems: Archives of Environmental Change, Balkema, p. 357-373.

Pastre J.-F., Leroyer Ch., LimondinLozouet N., Fontugne M., Hatte Ch., Krier V., Kunesch S. Et SAAd M.C.

2002 : «L'Holocène du Bassin parisien : variations environnementales et réponses géoécologiques des fonds de vallées » in RichaRd H., Vignot A. (DIR.), Équilibres et ruptures dans les écosystèmes durant les vingt derniers millénaires, Actes du colloque international de Besançon, septembre 2000, Besançon, P.U.F.C., Annales Littéraires 730, Série « Environnement, sociétés et archéologie 3 », p. 61-71.

Pastre J.-F., Leroyer Ch., LimondinLozouet N., Antoine P., Gauthier A., OrTh P.

2003 : «Quinze mille ans d'environnement dans le Bassin parisien (France) : mémoires sédimentaires des fonds de vallée », in Muxart T., Vivien F. -D., Villalba B., Burnouf J. (Dir.), Des milieux et des Hommes : fragments d'histoires croisées, Elsevier, p. 29-41.

Rameau J.-C., Mansion D., Dume G.

1989 : Flore forestière française : guide écologique illustré. T. 1 : Plaines et collines, Paris, Institut pour le dévelop- pement forestier, $1785 \mathrm{p}$.

REILLE M.

1990 : Leçons de Palynologie et d'analyse pollinique, Paris, C.N.R.S., 206 p.

\section{RICHARD H.}

1985 : «Un exemple de pollution anthropique dans les analyses polliniques : les habitats néolithiques du grand lac de Clairvaux (Jura). Palynologie archéologique », Actes des journées des 25-26-27 janvier 1984, CNRS-CRA, Notes et Monographies techniques, 17, p. 279-296.

\section{RICHARD H.}

1995 : «Analyse de l'anthropisation du milieu à partir de quelques exemples de variations de pollens d'arbres et d'arbustes. L'Homme et la dégradation de l'environnement », Actes des $X V^{c}$ Rencontres internationales d'archéologie et d'histoire d'Antibes, 20-22 octobre 1994, Juan-les-Pins, APDCA, p. $143-159$.

\section{STOCKMARR J.}

1972 : « Tablets with spores used in absolute pollen analysis », Pollen et Spores, 13 , p. 615-621.

\section{Triat-Laval H.}

1978 : Contribution pollenanalytique à l'histoire tardi- et postglaciaire de la végétation de la basse vallée du Rhône, Thèse de doctorat ès sciences, Université Aix-Marseille III, 343 p.

\section{Bibliographie malacologique}

\section{Limondin N.}

1992 : « Biogéographie holocène de Vertiginidae (Mollusca : Gastropoda) européens : relations avec la dernière déglaciation », Comptes-rendus de l'Académie des Sciences, 315 (II), p. 1281-1287.

\section{Puissegur J.-J.,}

1976 : Mollusques continentaux quaternaires de Bourgogne, Mémoires Géologiques de l'Université de Dijon, Paris, Doin, $241 \mathrm{p}$.

\section{BAHAIN J.-J.}

1997 : « Louvres "Le Bois d'Orville" (95 351001 AH) (Val-d'Oise), Compterendu de sondages géologiques » in Chopelain P. et al., Louvres " Le Bois d'Orville »(Val-d'Oise). Fouilles archéologiques d'un habitat du Haut Moyen-Age. Situation géographique et contexte géologique, archéologique et historique, D.F.S., S.R.A. d'Ile-deFrance, Conseil Général du Val-d'Oise, S.D.A.V.O., A.F.A.N., 1998, 25 p.

\section{BREZILLON M.}

1977 : « Informations archéologiques. Circonscription d'Île-de-France. Vald'Oise, Gonesse », Gallia Préhistoire, 
Paris, CNRS Éditions, 20/2, p. 354-355, 1 fig.

\section{Cecchini M.}

1990 : «Le Paléolithique en Pays de France », $B J P G F, 2,66$ p.

\section{KRIER V.}

2004 : La plaine alluviale de l'Oise : Milieu et système fluvial, stratigraphie des formations alluviales et archéologie $d u$ Tardiglaciaire à l'Holocène dans le Val-d'Oise. Saint-Ouen-L'Aumône, INRAP - Conseil général du Vald'Oise, direction de l'action culturelle, Service départemental d'archéologie, 2 tomes, $340 \mathrm{p}$.

\section{LEBRET P.}

1989 : «Tuf du Moulin de la Planche (commune de Gonesse - 95 - vallée du Crould) » in LeColle F. (Dir.), Les tufs et travertins quaternaires des bassins de la Seine et de la Somme et du littoral cauchois, essai d'inventaire, BCGC, Travaux du groupe Seine 4, novembre 1989, 37, non paginé.

Pastre J.-F., Cecchini M., Dietrich A., Fontugne M., Gauthier A., Kuzucuoglu C., Leroyer Ch., LiMONDIN N.

1991 : «L'évolution holocène des fonds de vallées au nord-est de la Région parisienne (France) : premiers résultats », Physio-Géo, 22-23, p. 109-115.

Référentiel pédologique, principaux sols d'Europe, Association Française pour l'Etude du Sol (AFES) et Institut National de Recherche Agronomique (INRA), 222 p, édition 1992.

\section{Bibliographie faunique}

Bemilli C.

2007. : « La faune du Néolithique final», in Brunet P., Cottiaux R. (Dir.), Meaux "Route de Varreddes, chemin de Flandres » (Seine-etMarne). Occupations du Néolithique sur les Coteaux de la Justice. RFO INRAP/DRAC Île-de-France, chap.9, p. 100-109.

Braguier S.

2007 : «Etude archéozoologique » in Praud Y., (DIR.), Rapport de fouille archéologique Houplin-Ancoisne (59) «Le Marais de Santes », Tome I, texte, p. 144-156.

\section{Horard-Herbin M.-P.}

1997 : «Le village celtique des Arènes à Levroux. Lélevage et les productions animales dans l'économie de la fin du second âge du Fer »12 $12^{\mathrm{e}}$ supplément à la $R A C F$, Levroux, FERACF/ADEL, $208 \mathrm{p}$.
Oueslati T.

2009 : «Approche archéozoologique de l'exploitation des ressources animales au Néolithique final », in DeCKers M., Delassus D. (Dir.), Valenciennes : vallée de l'Escaut. Un site du Néolithique final, p. 141-147.

\section{Bibliographie anthropologique}

\section{BARONE R.}

1977 : « Nomenclature anatomique française », in SOBOTTA J., Atlas d'Anatomie Humaine, Paris, Maloine, 4, $310 \mathrm{p}$

\section{Chabeuf M.}

1967 : « Les restes humains des tumulus de Villecomte (Côte-d'Or) », BMSAP, 1, XII ${ }^{\mathrm{e}}$ série, p. 459-475.

\section{Dechaume M.}

1966 : Précis de stomatologie, Paris, Masson et Cie, $4^{\mathrm{e}}$ éd.

\section{DUdAY H.}

1977 : « Le tumulus nº 1 de Sadoulet à Pompignan (Gard) ; étude des restes humains ", Cahiers d'Anthropologie, 2, p. 61-112.

Ferembach D., Schwidetsky I. eT StLoukal M.

1979 : «Recommandations pour déterminer l'âge et le sexe sur le squelette », BMSAP, 6, XIII' série, p. 7-45.

\section{HEIM J.-L.}

1963 : «Les apophyses géni, étude anthropologique », BMSAP, 4, $\mathrm{XI}^{\mathrm{e}}$ série, p. 585-658.

\section{Le Double A.-F.}

1906 : Traité des variations des os de la face de l'homme, et leur signification du point de vue de l'Anthropologie zoologique, Paris, Vigot, 471 p.

\section{MARTIN R.}

1957 : Lehrbuch der Anthropologie in systematischer Darstellung, Troisième édition, revue et corrigée par SALLER K., Stuttgart, Fischer, 2999 p.

\section{Masset C.}

1982 : Estimation de l'âge au décès par les sutures crâniennes, Thèse d'Etat ès sciences, Université de Paris VII, 301 p.

\section{Olivier G.}

1960 : Pratique anthropologique, Paris, Vigot, 291 p., 79 figures.

\section{RiQueT R.}

1970 : Anthropologie du Néolithique et du Bronze ancien, Poitiers, S.F.I.L. et Texier, $279 \mathrm{p}$.

\section{RIQUET R.}

1976 : « L’Anthropologie protohistorique française » in GuILAINE J. (Dir.) $L a$ Préhistoire Française, Paris, éd. du C.N.R.S., t. II, p. 135-152.

Bibliographie archéologique

Billard C. et al.

1994 : « Poses - Le Vivier - Le ClosSaint-Quentin (Eure). L'occupation de la plaine inondable au Néolithique et au début de l'Age du Bronze », RAO, 11, p. 53-113, 54 fig.

BLANCHET J.-C.

1984 : « Les premiers métallurgistes en Picardie et dans le Nord de la France. Chalcolithique, Age du Bronze et début du premier Age du Fer », MSPF, 17, $608 \mathrm{p}$.

Brezillon M.

1977 : « Informations archéologiques. Circonscription d'Ile-de-France. Vald'Oise. Gonesse », Gallia Préhistoire, CNRS Édition, Paris, 20, 2, p. 354-355.

Cottiaux R., Lawrence-Dubovac P.

2008 : «Un ensemble funéraire de l’Âge du Bronze moyen à Mareuil-lès-Meaux "Les Vignolles" (Seine-et-Marne) », RAIF, 1, p.113-124.

DuRAND J.

2007 : Le lithique in Meaux "Route de Varreddes, chemin des Flandres" (Seine-et-Marne). Occupations $d u$ Néolithique sur les "Coteaux de la Justice », RFO INRAP / DRAC Île-deFrance, ch. 7, p. 50-89.

Froquet-Uzel H.

2008 : «Les nécropoles à incinération du Bronze final, Courcelles (Loiret) : bilan préliminaire », BAPRAB, 5, p. 17-20.

Gouge P., Peake R.

2005 : « Aux marges du Bronze atlantique, sites et chronologies de la région du confluent Seine-Yonne », in Bourgeois J. et TAlon M. (éd.), L'Âge du Bronze du nord de la France dans son contexte européen, Actes du $125^{\text {ème }}$ congrès national des sociétés historiques et scientifiques, Lille 2000, CTHS / APRAB, Paris, p. 333-359.

Legriel J., Granchon P., Le Goff I., DE KePPer A.-G.

2010 : «La nécropole à incinération de l'âge du Bronze moyen de Cesson «Plaine du Moulin à Vent » (Seine-et-Marne) : premiers résultats ", BAPRAB, 7, p. 14-18.

\section{JACQUET P. (DIR.)}

1998 : Habitats de l'âge du Bronze à LyonVaise (Rhône), (coll.:Documents d'archéologie française $n^{\circ}$ 68), Paris, éd. MSH, 251 p., 174 fig. 
MARCigny C., Ghesquiere E. (Dir.)

2003 : L'île de Tatihou (Manche) à l'âge du Bronze. Habitats et occupation du sol, Paris, éd. MSH, (Coll.: Documents d'archéologie française $\mathrm{n}^{\circ}$ 96), $185 \mathrm{p}$.

\section{Marcigny C., Talon M.}

2009 : «Sur les rives de la Manche. Qu'en est-il du passage de l'Âge du Bronze à l'Âge du Fer à partir des découvertes récentes ? ", in De l'âge du Bronze à l'âge du Fer en France et en Europe occidentale ( $X^{e}-V I I^{e}$ siècle av. J.-C.). La moyenne vallée du Rhône aux âges du Fer. Actes du XXX colloque de l'A.F.E.A.F., Saint-Romain-en-Gal, 26-28 mai 2006. $27^{\mathrm{e}}$ suppl. à la $R A E$, Dijon, p. 385-403.

\section{Martial E.}

1995 : L'industrie lithique à l'âge du Bronze dans le Nord-Pas-de-Calais. Les exemples de Fréthun et de Roeux. Les Cahiers de Préhistoire du Nord, $n^{\circ} 15$, ler semestre 1994, Villeneuve d'Ascq 1995, 128 p., 91 fig.

\section{Pariat J.-G.}

2007 : Les sépultures in BRUNET P., Cottiaux R. (DIR.) Meaux « Route de Varreddes, chemin des Flandres » (Seine-et-Marne). Occupations du Néolithique sur les «Coteaux de la Justice ». RFO INRAP /DRAC Île-deFrance, ch. 4, p. 20-26, 9 fig.

\section{Martial E.}

1995 : «L'industrie lithique à l'âge du Bronze dans le Nord-Pas-de-Calais. Les exemples de Fréthun et de Roeux », $C P N, 15,1^{\text {er }}$ semestre 1994, Villeneuve d'Ascq 1995, 128 p.

\section{TARRETE J.}

1985 : « Informations archéologiques. Circonscription d'Île-de-France. Seine-et-Marne. Annet-sur-Marne, Les Plantards », Gallia Préhistoire, Paris, CNRS Éditions, 28, 2, p. 263-266.

\section{TARRETE J.}

1989 : «Informations archéologiques. Île-deFrance. Seine-et-Marne. Chelles. a) Le
Fossé de Travers Ouest », Gallia informations, Préhistoire et Histoire. Paris, CNRS Édition, 2, p. 33-35.

Villes A.

1987 : «Un aperçu de l'industrie lithique des niveaux de l'Age du Bronze au Fort Harrouard in Les relations entre le continent et les îles britanniques à l'Age du Bronze », Actes du colloque de Lille dans le cadre du $22^{e}$ Congrès préhistorique de France, 2-7 sept. 1984, SPF/ RAP, p. 275-305.

\section{VORUz J.-L.}

1996 : «Chronologie absolue de l'Âge $\mathrm{du}$ Bronze ancien et moyen » in Mordant C. et Gaiffe O. (Eds.), Cultures et sociétés du Bronze ancien en Europe. Actes du colloque «Fondements culturels, techniques, économiques et sociaux des débuts de l'âge du Bronze ".117e congr. Nat. Soc. Hist. Scient., Clermont-Ferrand, 27-29 oct. 1992, CTHS, Paris, p. 97-164. 